\author{
H. Bender
}

\title{
Die natürlichen Grundlagen der Mathematik
}

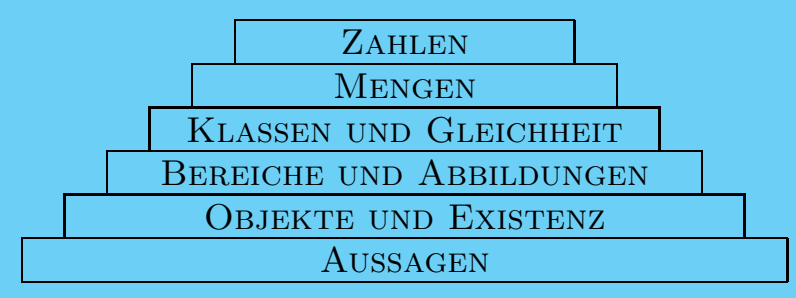





\section{Die natürLIChen Grundlagen DeR MathematiK}

Dargestellt im Geiste Dedekinds, Zermelos und Wittenbergs

von

Helmut Bender

Weil. o. Prof. für Mathematik an der Universität Kiel 
Bibliografische Information der Deutschen Nationalbibliothek

Die Deutsche Nationalbibliothek verzeichnet diese Publikation in der Deutschen Nationalbibliografie; detaillierte bibliografische Daten sind im Internet über http://dnb.d-nb.de abrufbar.

3., neu bearbeitete Auflage

(C) Copyright Logos Verlag Berlin GmbH 2015, 2017, 2020

Alle Rechte vorbehalten.

ISBN 978-3-8325-4072-2

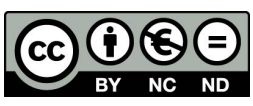

Logos Verlag Berlin GmbH

Comeniushof, Gubener Str. 47, 10243 Berlin

Tel.: +49 (0)3042851090

Fax: +49 (0)3042851092

INTERNET: http://www.logos-verlag.de 


\section{INHALT}

Vorwort und Einführung

I. Aufbau des Zahlensystems

1. Vervollständigung archimedisch und dicht geordneter Gruppen . . . 10

2. Vervollständigung archimedisch geordneter Körper.............. 12

3. Quotientenkörper archimedisch geordneter Ringe ............. 14

4. Geordnete Mengen, Gruppen und Ringe vom Typ $\mathbb{Z} \ldots \ldots \ldots \ldots 16$

5. Wohlgeordnete Mengen und der $f$-Kettensatz $\ldots \ldots \ldots \ldots \ldots \ldots \ldots$

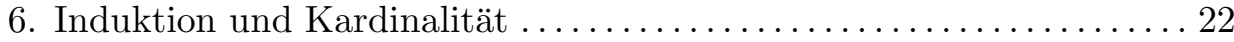

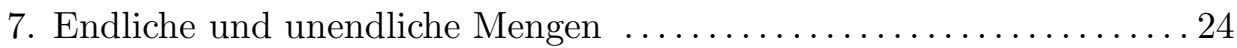

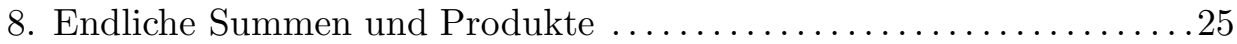

Literaturhinweise

II. Dedekind und die Grundlagen

1. Dedekinds Brief an Dr. Hans Keferstein vom 27. Februar 1890 . . . 27

2. Dedekinds Zahlenschrift - Eckstein und Stein des Anstoßes . ..... 41

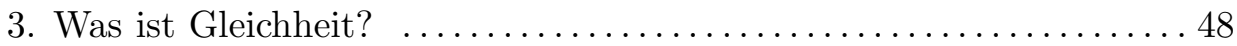

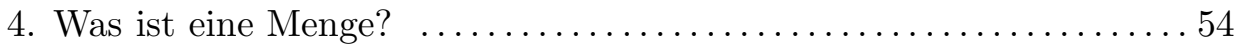

5. Existenz - Negation - Eigenschaften . . . . . . . . . . . . . . 61

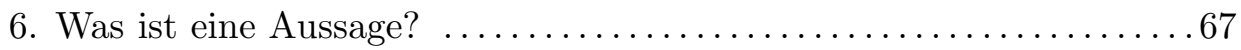

III. Bereiche - Klassen - Mengen

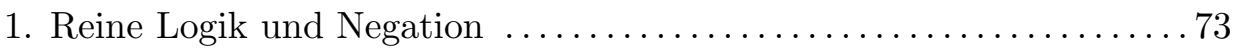

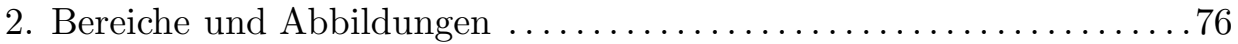

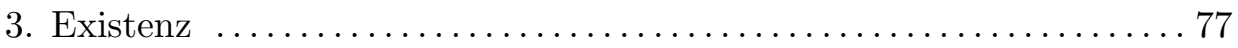

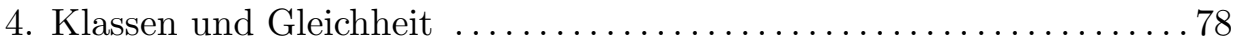

5. Existenz von Teilklassen und Abbildungen $\ldots \ldots \ldots \ldots \ldots \ldots \ldots \ldots$

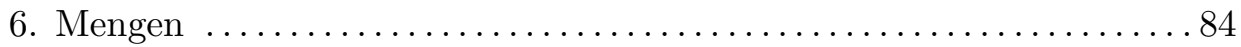

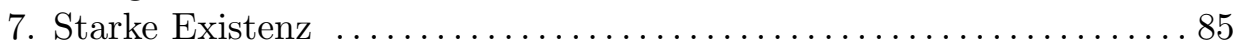

8. Existenz multivariabler Funktionen $\ldots \ldots \ldots \ldots \ldots \ldots \ldots \ldots \ldots \ldots$

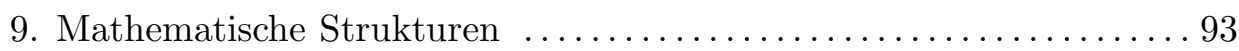

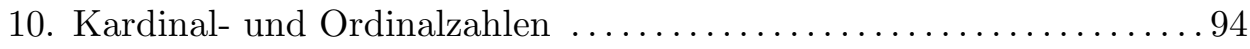

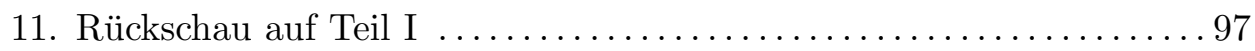

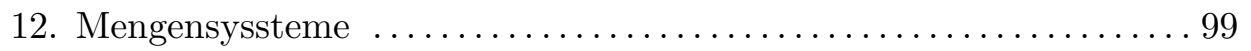

Literatur 



\section{Vorwort.}

Wie die zweite, so verdankt auch die dritte Auflage ihre Existenz nicht exorbitanten Verkaufszahlen, sondern einem Erkenntnisfortschritt:

Die logische Regel 1.5(a) in Teil III ist neu. Mein Inneres hatte sich lange dagegen gesträubt, sie wahrzunehmen. Denn sie vereinfacht via 1.7(a)(b) vorher nur eine Kuriosität - die logischen Verhältnisse in einem sogenannten Standardbeweis auf geradezu dramatische Weise [1.10], und so manch schöner Gedanke wurde dadurch hinfällig.

Damit könnte ich es bewenden lassen, ergreife aber die Gelegenheit, mich aus einer gewissen Distanz heraus zu meinem „Aufbau des Zahlensystems“ zu äußern. ${ }^{1}$ Auch aus einem gewissen Pflichtgefühl heraus, als Warnung: Meine Darstellung enthalte „kaum Neues“, so ein Bescheid von Expertenseite (2012).

Nicht nur aus einem gewissen missionarischen Eifer ${ }^{2}$ heraus hatte ich die Sache (inklusive Kefersteinbrief) damals an eine Zeitschrift geschickt, sondern auch um auf diese Weise explizit von wesentlichen Übereinstimmungen mit der Literatur zu erfahren. Derartiges hätte mich auch nicht im mindesten überrascht, ist es doch mehr als naheliegend, etwa die additive Gruppe von „ $\mathbb{Z}$ " als Automorphismengruppe einer geordneten Menge vom Typ $\mathbb{Z}$ zu gewinnen, damit auch gleich eine geordnete Gruppe vom Typ $\mathbb{Z}$, und dann ganz analog den Ring „, $\mathbb{Z} “$ (plus Ordnung) als Endomorphismenring.

Letzteres findet sich denn auch in W. Felschers Werk Naive Mengen und abstrakte Zahlen ${ }^{3}$. Auch er bemüht sich um eine eher begriffliche als rechnerische Vorgehensweise. Daraus resultieren Themen wie wohlgeordnete Mengen und Vervollständigung geordneter Gruppen.

Auch das Buch Zahlen ${ }^{4}$ von H. D. Ebbinghaus, H. Hermes, F. Hirzebruch, M. Koecher, K. Mainzer, A. Prestel und R. Remmert habe ich inspiziert. Der Aufmerksamkeit des Lesers seien besonders die Abschnitte über die Addition und Multiplikation in " $\mathbb{R}$ " empfohlen.

Sie führen zu E. Landaus klassischem Opus Grundlagen der Analysis ${ }^{5}$, rein rechnerisch und detailliert bis zum Exzeß. Lange Zeit wird nur im Positiven agiert, der historischen Entwicklung entsprechend wie der eines Individuums. Erst wenn die positiven „reellen Zahlen" hinsichtlich Addition, Multiplikation und Ordnung vorliegen, wird neben der 0 jedem $r$ sein Zwilling $-r$,erschaffen“.

Das erinnert an Dedekinds Schöpferkraft des menschlichen Geistes, und es erinnert mich an eine frühere Version meines Opus:

\footnotetext{
${ }^{1}$ Die Gänsefüßchen deuten an, daß ich diesen gängigen Titel als reinen terminus technicus verstehe. Ich rede nie von "Zahlen“, sondern nur von geordneten Mengen, Gruppen, Ringen und Körpern.

${ }^{2}$ Seht, so erledigt man die Dinge im 21. Jahrhundert!

${ }^{3}$ B.I.-Wissenschaftsverlag, Mannheim, ... 1978.

${ }^{4}$ Springer-Verlag, Berlin, ... 1983.

${ }^{5}$ Akademische Verlagsgesellschaft, Leipzig 1930.
} 
Um aus einer geordneten Menge $N$ vom Typ $I N$ eine solche vom Typ $\mathbb{Z}$ zu gewinnen, wird die Menge $Z$ aller Paare $(x, y)$ mit $x, y \in N$ und $x=m$ oder $y=m$ betrachtet, wobei $m=\min (N)$. Dann wird $(x, m) \leq(m, y)$ festgesetzt, ferner $(m, a) \leq(m, b) \Leftrightarrow a \leq b$ und $(a, m) \leq(b, m) \Leftrightarrow b \leq a$.

Man denke bei $m$ an 0 , bei $(m, x)$ an $x$, bei $(x, m)$ an $-x$.

Genauso hätte Landau verfahren können, nur die 0 müßte vorher seinen Zahlen „hinzugefügt“ werden. Warum bin ich den komplizierteren Weg in 5.10 gegangen? Um Paarmengen weitmöglichst aus dem Weg zu gehen. ${ }^{6}$

Irgendwann muß der Sprung hinab ins Negative getan werden. Landau springt so spät wie möglich, ich so früh wie möglich.

Anstatt mit den etwas unhandlichen „Schnitten“ im ursprünglichen Sinne Dedekinds, wird generell entweder mit unteren Abschnitten ohne Maximum gearbeitet, oder mit oberen Abschnitten ohne Minimum.

Bei oberflächlicher Betrachtung macht es keinen Unterschied.

Der Witz bei meinem Vorgehen liegt aber gerade darin, daß die im Positiven liegenden oberen Abschnitte eines geordneten Körpers $K$ zugleich die oberen Abschnitte der aus den Elementen $>0$ bestehenden multiplikativen Gruppe $K_{0}$ sind. Wie ich wendet Felscher die Gruppenvervollständigung auch auf $K_{0}$ an, nicht nur auf die additive Gruppe $K^{+}$, und vermeidet dadurch langatmige Rechnungen. Aber leider arbeitet er wie Landau mit unteren Abschnitten; das mindert den Synergieeffekt.

Warum setze ich den eigentlich letzten Bauabschnitt, den Übergang von $Q$ zu $I R$, an den Anfang? Der Klarheit halber, zwecks Konzentration auf das wesentliche. Nichts kommt ins Spiel, was irgendwie $I N$ involviert.

Wie mir erst jetzt auffiel, reicht Dedekind's „Rekursionssatz" [7, 126], ein Spezialfall von I.6.3 ( $f$ konstant), nicht aus um das Thema induktive Definition abzudecken. Man braucht nur an die Fibonaccifolge zu denken, oder an induktiv definierte geordnete Produkte.

Den Kollegen A. Chermak und B. Stellmacher danke ich für ihr Interesse und wertvolle Gespräche.

Besonderen Dank schulde ich Herrn Buchholtz vom Logos Verlag.

Kiel, im Dezember 2019

\footnotetext{
${ }^{6}$ Was ist überhaupt ein Paar?

Der kundige Leser: Das hat uns doch Kuratowski gelehrt!

Ich: Lieber ließe ich mich kreuzigen ...
} 


\section{Einführung.}

Die Elimination des naiven Konvergenzbegriffs, mittels expliziter Definition, ist der erste wirkliche Beitrag zum Thema Grundlagen der Mathematik.

Analog eliminiert Dedekind das Endliche, mitsamt natürlichen Zahlen, Induktion, Iteration, Folgen; auf einer neuen begrifflichen Basis, Mengen und Abbildungen, wie sie de facto bis heute der Mathematik zugrundeliegen.

Elimination heißt Vergessen plus Rekonstruktion des Vergessenen in einer Synthese, mit geeigneten Begriffsbildungen und Annahmen, nahegelegt durch eine vorausgehende Analyse der Mathematik, so wie sie sich dem objektiven Auge darbietet.

Was nicht mehr dem Vergessen anheimfallen kann, weil die Rekonstruktion ohne das Vergessene nicht zu bewältigen ist, nenne ich Reine Logik.

In diesem Restbestand mathematischer Sprachkultur äußern sich Menschen in Aussagen über Aussagen, allein mit den Worten und, oder, wenn/dann und ihren Synonymen. Darin werden dann die mathematischen Grundbegriffe verankert, Objekt, Bereich, Abbildung. Sie sind also rein sprachlicher Natur.

Wie bei den Beispielen Konvergenz und Endlichkeit stellt sich schließlich die Frage, ob und wie das Vergessene sich auf der tieferen Grundlage wieder rekonstruieren läßt, und weil nun alles auf einmal vergessen wurde, steht somit die gesamte real existierende Mathematik auf dem Prüfstand. Eigentlich wie bei Dedekind, denn schon das Endliche durchzieht die gesamte Mathematik.

Die der Synthese in Teil III vorausgehende Analyse findet in Teil II statt, im Rahmen einer Gesamtschau auf das Grundlagenthema. Vorbild ist Dedekinds Analyse der „Zahlenreihe $N$ " im Kefersteinbrief [II.1]; und seine Synthese, der "Aufbau" des Zahlensystems, findet sich in Teil I, in zeitgemäßem algebraischen Gewande, in einer gewissen Abrundung.

Ich setze natürlich eine gewisse Vertrautheit mit dem voraus, dessen Grundlagen hier „tiefergelegt" werden sollen, mit der „real existierenden“ Mathematik also, kurz r. e. M. genannt, gelegentlich auch Cantors Paradies.

Vor allem wende ich mich an Mathematiker, die mit ihrem Verständnis der logischen und begrifflichen Grundlagen ihres Faches nicht ganz zufrieden sind.

Gewidmet sei dies Büchlein dem Andenken an die deutsche Universität. 



\section{Aufbau Des Zahlensystems}

Nach einer im WS 2005/06 an der Universität Kiel gehaltenen Vorlesung

Gebt mir eine unendliche Menge, und ich gebe euch eure Zahlen mit allem was dazugehört.

Mit diesen Worten hätte Dedekind seinen Beitrag zum Thema Zahlen beschreiben können.

1872: Stetigkeit und irrationale Zahlen.

1888: Was sind und was sollen die Zahlen?

Das klarzumachen ist die Aufgabe, und zwar so, wie Dedekind es uns aufgegeben hat: auf der Basis natürlicher Allgemeinbegriffe, das begriffliche Schließen dem rechnerischen vorziehend, das Innerliche dem Äußerlichen in der Mathematik.

Dedekind erwartet von seinen Lesern „außer dem gesunden Menschenverstande noch einen sehr starken Willen, um Alles vollständig durchzuarbeiten“.

Statt eines starken Willens, der in der Tat manchmal nötig sein mag, um sich durch langweilige Rechnungen zu quälen, setze ich Begriffe wie Gruppe, Ring, Körper, geordnete Menge, Automorphismengruppe, Endomorphismenring voraus, mit ihrem unmittelbaren Umfeld, und neben dem üblichen Umgang mit Mengen und Abbildungen natürlich, welche von Dedekind erstmals explizit einer mathematischen Betrachtung zugrunde gelegt wurden.

Die angesprochene Begriffswelt etablierte sich erst im Laufe des 20. Jahrhunderts im mathematischen Denken, und in ihr lassen sich die Dinge in ihrer natürlichen Einfachheit darstellen.

Ein wichtiger Aspekt ist das Prinzip, daß isomorphe Strukturen dieselben Eigenschaften haben, ein Isomorphismus $\alpha: X \rightarrow Y$ zu $X$ gehörige Objekte $A, B, C, \ldots$ auf analog zu $Y$ gehörige Objekte $A \alpha, B \alpha, C \alpha, \ldots$ mit denselben Eigenschaften abbildet, wie umgekehrt der Isomorphismus $\alpha^{-1}: Y \rightarrow X$.

Folgendes ist als ein ganz gewöhnliches Stück moderner Mathematik anzusehen, eine Ansammlung von Definitionen, Sätzen und Beweisen; mit der kleinen Einschränkung natürlich, daß nirgends von „Zahlen“ die Rede ist und nichts einen anschaulichen Endlichkeitsbegriff involviert. Auf Historisches und Mathematikphilosophisches wird später eingegangen. 
1. Vervollständigung archimedisch und dicht geordneter Gruppen.

1.1. In diesem $\S 1$ ist $G$ eine geordnete Gruppe, d.h. eine abelsche Gruppe $G \neq 0(\neq 1$ bei multiplikativer Notation) zusammen mit einer

$$
x \leq y \quad \Rightarrow \quad x+a \leq y+a,
$$

genügenden linearen Ordnungsrelation $\leq$.

FOLGERUNGEN.

(a) Für jedes $a \in G$ ist die Abbildung $x \rightarrow x+a$ ein Automorphismus der geordneten Menge $G: x \leq y \Leftrightarrow x+a \leq y+a$.

(b) Und $x \rightarrow-x$ ist ein Anti-Automorphismus: $x \leq y \Leftrightarrow-x \geq-y$.

(c) Genau dann ist also $a$ eine obere Schranke einer Teilmenge $U$, wenn $-a$ eine untere Schranke von $-U=\{-u \mid u \in U\}$ ist, und dasselbe gilt für Maximum/Minimum wie Supremum/Infimum. Eine Untergruppe $U \neq 0$ hat kein Maximum, nicht einmal ein Supremum, denn zu $s=\sup (U)$ und $0<u \in U$ existiert $x \in U$ mit $s-u<x$, also $s<x+u \in U$.

(d) $s \leq x, t \leq y \Rightarrow s+t \leq x+y$.

\subsection{BeZEICHNUNGEN.}

$G$ dicht: $\mathrm{Zu} a<b$ existiert $u$ mit $a<u<b$.

$U$ dicht in $G: u$ kann immer in $U(\subseteq G)$ gewählt werden.

$G$ archimedisch: Untergruppen $\neq 0$ sind unbeschränkt.

Gleichwertig: Unterhalbgruppen $\neq 0$ sind unbeschränkt.

$G$ vollständig: Jede unten beschränkte Teilmenge $\neq \emptyset$ hat ein Infimum.

Gleichwertig: Jede oben beschränkte Teilmenge $\neq \emptyset$ hat ein Supremum.

$\operatorname{Min}(X)$ ist die Menge aller Minima von $X(\subseteq G)$, und $\min (X)$ ist das Minimum von $X$ falls $\operatorname{Min}(X) \neq \emptyset$ (analog: Max/max, Sup/sup, Inf/inf).

$X+a=\{x+a \mid x \in X\}$ und $X+Y=\{x+y \mid x \in X, y \in Y\}$.

$G_{a}=G_{>a}=\{x \in G \mid x>a\}$.

$A \subseteq G$ ist ein (oberer) Abschnitt von $G \Longleftrightarrow A \supseteq G_{a}$ für alle $a \in A$.

$A$ ist speziell, wenn zusätzlich $\emptyset \neq A \neq G$ und $\operatorname{Min}(A)=\emptyset$.

Gleichwertig: $G \backslash A(\neq \emptyset, G)$ ist die Menge der unteren Schranken von $A$.

$\mathrm{S}(G)$ ist die Menge aller speziellen Abschnitte.

$\mathcal{P}(M)$ ist die Menge aller Teilmengen einer Menge $M$.

Statt geordnete Gruppe sage ich auch Geogruppe (analog für geordnete Mengen, Ringe, Körper), Halbgruppen und Ringe haben ein Einselement, und ein Integritätsring ist kommutativ, nullteilerfrei und $\neq 0$.

Jede Teilmenge einer Geomenge $G$ ist bezüglich der gegebenen Ordnung wiederum eine Geomenge, sozusagen eine Untergeomenge von $G$.

In $\S 5$ bekommt $G_{a}$ wie Abschnitt eine andere Bedeutung (die duale).

Soweit sinnvoll, gilt alles in 1.2 und 1.3 für beliebige lineare Geomengen. 


\subsection{Bemerkungen.}

(a) Für Abschnitte $X, Y$ gilt $X \subseteq Y$ oder $Y \subseteq X$.

(b) Eine Vereinigung von Abschnitten ist ein Abschnitt.

(c) Eine Vereinigung $\neq G$ von speziellen Abschnitten ist speziell.

(d) Bezüglich $\subseteq$ ist $\mathrm{S}(G)$ linear und vollständig [(a)(c)].

(e) Mit $X$ ist auch $X+a$ speziell [1.1(a)].

(f) Mit $X, Y$ ist auch $X+Y$ speziell [(e)(c)1.1(d)].

(g) $X+a=X+G_{a}$ in (e) $\left[x+a=x^{\prime}+\left(a+x-x^{\prime}\right)\right.$ mit $\left.x^{\prime}<x\right]$.

(h) Ist $G$ dicht, so ist jedes $G_{a}$ speziell und

$$
G_{a}+G_{b}=G_{a+b}
$$

für alle $a, b \in G\left[g>a+b \Longrightarrow g=\left(g-g^{\prime}\right)+g^{\prime}\right.$ mit $\left.g-a>g^{\prime}>b\right]$.

(i) Für spezielle $X \supset Y$ und $a<b$ in $X \backslash Y$ gilt

$$
X \supset G_{a} \supset G_{b} \supseteq Y \text {. }
$$

(j) $G$ sei dicht und $X$ speziell. Aufgrund 1.1(b) ist dann auch

$$
Y=-(G \backslash X \backslash \operatorname{Max}(G \backslash X))
$$

speziell, denn $-Y$ ist ein unterer Abschnitt $\neq \emptyset, G$ ohne Maximum.

1.4. Satz. $G$ sei dicht und archimedisch.

Bezüglich Addition [1.3(f)] und $X \leq Y \Leftrightarrow X \supseteq Y$ ist $\mathrm{S}(G)$ eine vollständige Geogruppe, $\gamma: a \rightarrow G_{a}$ ist ein Monomorphismus von $G$ in $\mathrm{S}(G)$, und das Bild $G^{\gamma}$ ist dicht in $\mathrm{S}(G)$.

Beweis. $G_{0}$ ist Nullelement [1.3(gh)], und mit $G$ ist auch die Addition von Teilmengen assoziativ und kommutativ. Sei $X$ speziell und $Y$ wie in 1.3(j). Behaupte $X+Y=G_{0}$. Betrachte $x \in X$ und $y \in Y$.

Wegen $-y \notin X$ ist $-y<x$, d.h. $x+y \in G_{0}$. Nehme also $X+Y \subset G_{0}$ an.

Wähle $a, b \in G_{0} \backslash X+Y$ mit $a<b$, also $a<b<x+y$, folglich $a-y<$ $b-y<x$. Daher ist $a-y$ eine nichtmaximale untere Schranke von $X$, d.h. $y-a$ liegt in $Y$. Es folgt $Y \subseteq Y+a$, und wegen $a \geq 0$ ist auch $Y+a \subseteq Y$.

Die Untergruppe $H=\{h \in G \mid Y+h=Y\}$ von $G$ ist somit $\neq 0$. Wegen $y+H \subseteq Y$ ist sie (wie $Y$ ) unten beschränkt, ein Widerspruch [1.1(c)].

Damit ist $\mathrm{S}(G)$ eine abelsche Gruppe. Die Regeln $a<b \Rightarrow G_{a} \supset G_{b}$ und $X \supseteq Y \Rightarrow X+A \supseteq Y+A$ sind trivial. Das übrige findet sich in 1.3(dhi).

1.5. Zusatz. Ist $H$ eine vollständige Geogruppe (nach 1.1(c) archimedisch) und $G$ eine in $H$ dichte Untergruppe, so ist die Abbildung $a \rightarrow G_{a}=H_{a} \cap G$ ein Isomorphismus von $H$ auf $\mathrm{S}(G)$ [1.3(h) gilt für alle $a, b \in H]$.

1.6. Folgerung. $G^{\prime}$ und $H^{\prime}$ seien wie $G$ und $H$.

Ein Isomorphismus $\varphi: G \rightarrow G^{\prime}$ läßt sich dann zu einem (und nur einem) Isomorphismus $H \rightarrow H^{\prime}$ fortsetzen. Dieser bildet $a \operatorname{auf} \inf \left(G_{a} \varphi\right)$ ab. 


\section{Vervollständigung archimedisch geordneter Körper.}

2.1. In diesem $\S 2$ ist $K$ ein geordneter Körper, d. h. ein Körper $K$ zusammen mit einer linearen Ordnungsrelation $\leq$ auf $K$, derart daß aus $x \leq y$ immer $x+a \leq y+a$ folgt sowie $x a \leq y a$ für $a>0$, d.h. für $a, b>0$ ist auch $a b>0$.

Dann ist die additive Gruppe $K^{+}$eine Geogruppe, ebenso der Abschnitt $K_{0}=\{a \in K \mid a>0\}$ bezüglich Multiplikation.

Neben den Automorphismen $x \rightarrow x+a(a \in K)$ hat die Geomenge $K$ auch noch die Automorphismen $x \rightarrow x a(a>0)$. Ihre Automorphismengruppe ist daher transitiv auf der Menge der Paare $(x, y)$ mit $x<y$ :

Durch Addition von $-x$ erhalten wir nämlich aus $(x, y)$ ein Paar $(0, z)$ mit $0<z$, und Multiplikation mit $z^{-1}$ führt dann zu $(0,1)$.

Insbesondere ist $K$ dicht [es gibt $x<u<y$ in $K$ ], d.h. $K^{+}$ist dicht, und als Abschnitt von $K$ ist auch $K_{0}$ dicht.

Natürlich bezieht sich archimedisch auf $K^{+}$, wie später bei Georingen.

Vollständige Geokörper sind also archimedisch [1.1(c)].

2.2. Lemma. Sei $L$ eine Geogruppe und $\alpha: L_{0} \rightarrow L_{0}$ mit

$$
(u+v) \alpha=u \alpha+v \alpha \quad \text { für alle } u, v \in L_{0} .
$$

Setze $0 \alpha=0$ und $(-u) \alpha=-u \alpha$ für $u \in L_{0}$.

Dann gilt (*) für alle $u, v \in L$.

Beweis. Für $x, y \in L$ ist $(x+y) \alpha=x \alpha+y \alpha$ nachzuweisen. Für $x=0$ wie $y=0$ ist das trivial. Außerdem ist $(-z) \alpha=-z \alpha$ für alle $z \in L$.

Für $x+y \geq 0$ und etwa $x>0, y<0$ folgt

$$
x \alpha=((x+y)+(-y)) \alpha=(x+y) \alpha+(-y) \alpha=(x+y) \alpha-y \alpha,
$$

und das erledigt auch den Fall $x+y \leq 0$ :

$$
(x+y) \alpha=-(-x-y) \alpha=-((-x) \alpha+(-y) \alpha)=-(-x \alpha-y \alpha) .
$$

2.3. Folgerung. Sei $L$ eine Geogruppe.

Auf $L_{0}$ sei eine Multiplikation gegeben, die $L_{0}$ zu einer Geogruppe macht und der Regel $(u+v) a=u a+v a$ genügt. Setze zusätzlich

$$
0 x=0=x 0 \quad(-u) a=-u a=a(-u) \quad(-u)(-a)=u a
$$

für $a, u \in L_{0}$ und $x \in L$. Dann ist $L$ ein Geokörper.

2.4. Hilfssatz. Sei auch $L$ ein Geokörper und $\gamma: K^{+} \rightarrow L^{+}$ein Gruppenhomomorphismus mit $(u v)^{\gamma}=u^{\gamma} v^{\gamma}$ für alle $u, v \in K_{0}$.

Dann gilt das sogar für alle $u, v \in K$ [wegen $(-x)^{\gamma}=-x^{\gamma}$ und $0^{\gamma}=0$ ].

2.5. Hilfssatz. Für Abschnitte $X, Y, A \subseteq K_{0}$ gilt

$$
(X+Y) A=X A+Y A \text {. }
$$

Beweis. Die Inklusion $(X+Y) A \subseteq X A+Y A$ ist völlig trivial, und die Umkehrung ergibt sich aus $x a+y b=x a+y \frac{b}{a} a$ mit $y \frac{b}{a} \geq y$ falls $a \leq b$. 


\subsection{Lemma. Mit $K$ ist auch $K_{0}$ archimedisch.}

Beweis. Nehme eine beschränkte Untergruppe $U \neq 1$ von $K_{0}$ an. Die Menge $A$ aller $a \geq 0$, zu denen ein $a^{\prime} \in U$ mit $a^{\prime} \geq 1+a$ existiert, ist dann ebenfalls beschränkt. Für $1 \leq u \in U$ ist $u-1 \in A$, und es gibt ein $u>1$.

Wegen $a^{\prime} b^{\prime} \geq(1+a)(1+b)=1+a+b+a b \geq 1+a+b$ ist $A$ additiv abgeschlossen, ist also eine beschränkte Unterhalbgruppe $\neq 0$ von $K^{+}$(und die Menge aller $a-b$ mit $a, b \in A$ wäre eine beschränkte Untergruppe $\neq 0$ ).

2.7. SAtz. Ist $K$ archimedisch, so existiert ein Monomorphismus in einen vollständigen Geokörper.

Genauer erhalten wir aus Satz 1.4 die Geogruppen $L=\mathrm{S}\left(K^{+}\right)$und $\mathrm{S}\left(K_{0}\right)$ sowie $\gamma: K \rightarrow L$, zugleich monomorph von $K^{+}$in $L$ und von $K_{0}$ in $\mathrm{S}\left(K_{0}\right)$.

Als Menge ist $\mathrm{S}\left(K_{0}\right)=L_{0}$. Über 2.6 und 2.3 entsteht nun der Geokörper $L=\mathrm{S}(K)$, und aufgrund 2.4 ist $\gamma$ sogar ein Körpermonomorphismus.

Außerdem ist $K \gamma$ dicht in $L$.

2.8. ZuSATZ. 1.5/6 gilt analog für Geokörper.

Dazu führt zweifache Anwendung von 1.5/6, kombiniert mit 2.4.

2.9. Lemma. In einem vollständigen Geokörper ist jeder Teilkörper dicht.

Beweis. $K$ sei vollständig und der Teilkörper $L$ nicht dicht in $K$.

Dann existieren $a, b \in K$ mit $a<b$ und

$$
a<u<b \Rightarrow u \notin L .
$$

Da $K$ archimedisch ist, existiert $x \in L$ mit $x \leq a$.

Da auch $K_{0}$ archimedisch ist [2.6], existiert $y \in L_{0}$ mit $y<b-a$.

Wähle $k \in K$ mit $k<x$ für ein $x$. Sei $X$ die Menge aller $x>k$.

Die Menge $H$ aller $h \in K$ mit $X+h \subseteq X$ ist wegen $X \neq \emptyset$ beschränkt und ist eine Unterhalbgruppe von $K^{+}$, ist also $=0$.

Jedes $y$ liegt jedoch in $H$, denn für jedes $x \in X$ ist zum einen $x+y>x>k$, zum andern $x+y<b$, wegen $(*)$ also $x+y \leq a$, insgesamt also $x+y \in X$.

Nachbemerkung. Von einem Iso/Monomorphismus $\alpha: G \rightarrow H$ zwischen Geomengen $G, H$ wird neben bijektiv/injektiv noch

$$
x \leq y \quad \Longleftrightarrow x \alpha \leq y \alpha
$$

für alle $x, y \in G$ verlangt. Ein Monomorphismus ist also ein Isomorphismus auf das Bild. Bei linearem $G$ genügt $\Rightarrow$, im allgemeinen aber nicht. Die Verhältisse sind also denen in der reinen Algebra, wo ein Iso/Monomorphismus einfach ein bijektiver/injektiver Homomorphismus ist, nicht ganz analog. 


\section{Quotientenkörper archimedisch geordneter Ringe.}

3.1. Ein geordneter Ring ist ein Integritätsring zusammen mit einer linearen Ordnungsrelation $\leq$ wie in 2.1 .

3.2. Ist der Integritätsring $A$ Teilring eines Körpers $K$ - allgemeiner eines Ringes $K$, in dem jedes Element $\neq 0$ von $A$ invertierbar ist - so ist die Menge aller Quotienten $\frac{a}{b}=a b^{-1}=b^{-1} a$ mit $a, b \in A$ (und natürlich $b \neq 0$ ) aufgrund

$$
\frac{x}{y}+\frac{u}{v}=\frac{x v+y u}{y v} \quad \frac{x}{y} \frac{u}{v}=\frac{x u}{y v} \quad-\frac{x}{y}=\frac{-x}{y} \quad\left(\frac{x}{y}\right)^{-1}=\frac{y}{x}
$$

ein Teilkörper von $K$, der Quotientenkörper $\mathrm{Q}(A)=\mathrm{Q}_{K}(A)$ von $A$ in $K$.

Diese Regeln, plus $\frac{x}{y}=\frac{u}{v} \Leftrightarrow x v=u y$, zeigen auch, daß ein Isomorphismus $\alpha: A \rightarrow A^{\prime}$ durch $\frac{x}{y} \rightarrow \frac{x \alpha}{y \alpha}$ zu einem Isomorphismus zwischen $\mathrm{Q}(A)$ und (analogem) $\mathrm{Q}\left(A^{\prime}\right)$ fortgesetzt wird.

3.3. Sei $A$ in 3.2 geordnet und $K=\mathrm{Q}(A)$. Dann ist die Menge $P$ aller $\frac{a}{b}$ mit $a, b>0$ ein Positivitätsbereich von $K$, d.h. $P$ ist additiv und multiplikativ abgeschlossen, mit $K=P \dot{\cup}\{0\} \dot{\cup}\{-P\}$.

Durch $k<k^{\prime}: \Leftrightarrow k^{\prime}-k \in P$ wird $K$ dann ebenfalls geordnet, und diese Ordnung stimmt auf $A$ mit der alten Ordnung überein.

\subsection{Lemma. Mit $A$ ist in 3.3 auch $K$ archimedisch.}

Beweis. Sei $\frac{a}{b}(b>0)$ eine obere Schranke der Untergruppe $U \neq 0$ von $K^{+}$. Da $A b$ in $A$ unbeschränkt ist, existiert $c \in A$ mit $c b>a$, d.h. $c>\frac{a}{b}$.

Sei $0 \neq \frac{x}{y} \in U(y>0)$. Dann ist $A \cap U y$ eine (oben durch $c y$ ) beschränkte Untergruppe $\neq 0$ von $A^{+}$, ein Widerspruch.

\subsection{Satz. Sei $A$ ein Integritätsring und $V$ ein torsionsfreier $A$-Modul.}

Dann gibt es einen Monomorphismus von $V$ in einen Modul $W$ der Art, daß der Endomorphismus $a_{W}: w \rightarrow w a$ von $W$ für $0 \neq a \in A$ bijektiv ist.

Beweis. Für jede Menge $X$ ist der aus allen $f: X \rightarrow V$ bestehende „Funktionenraum" $\mathcal{F}(X, V)$ bezüglich

$$
f+g: x \rightarrow x f+x g \text { und } f a: x \rightarrow(x f) a .
$$

ein $A$-Modul, und im Falle eines $A$-Moduls $X$ ist $\operatorname{Hom}(X, V)$ ein Teilmodul.

Für jedes $f \in F=\mathcal{F}(A, V)$ sei $\mathcal{A}_{1}(f)$ bzw. $\mathcal{A}_{0}(f)$ die Menge aller Ideale $X \neq 0$ von $A$ mit $f_{\mid X} \in \operatorname{Hom}(X, V)$ bzw. $f_{\mid X}=0$.

Und $F_{i}(i=0,1)$ sei die Menge aller $f$ mit $\mathcal{A}_{i}(f) \neq \emptyset$. 
Mit $X$ und $Y$ ist auch $X \cap Y$ ein Ideal $\neq 0[x, y \neq 0 \Rightarrow x y \neq 0]$. Also ist

(*) $\quad \mathcal{A}_{i}(f) \cap \mathcal{A}_{j}(g) \neq \emptyset$ für $f \in F_{i}$ und $g \in F_{j}$,

und somit sind $F_{0}$ und $F_{1}$ Teilmoduln von $F$.

Setze $W=F_{1} / F_{0}$ (Faktormodul).

Torsionsfrei bedeutet $v a \neq 0$ für alle $v \neq 0$ und $a \neq 0$. Das impliziert

$(* *) \quad \mathcal{A}_{0}(f a)=\mathcal{A}_{0}(f)$ für $a \neq 0$.

Für $v \in V$ ist $f_{v}: a \rightarrow v a$ ein Modulhomomorphismus (ebenso $v \rightarrow f_{v}$ ).

Insbesondere liegt $f_{v}$ in $F_{1}\left[A \in \mathcal{A}_{1}\left(f_{v}\right)\right]$, in $F_{0}$ aber nur wenn $v=0$.

Die Abbildung $v \rightarrow F_{0}+f_{v}$ ist somit ein Monomorphismus von $V$ in $W$.

Für $0 \neq a \in A$ ist $a_{V}$ bijektiv von $V$ auf $V a$. Sei $\beta: V a \rightarrow V$ das Inverse.

Für $f \in F_{1}$ und $X \in \mathcal{A}_{1}(f)$ ist $X a f=X f a \subseteq V a$.

Erhalte so den Homomorphismus $f_{\mid X a} \beta: X a \rightarrow V$ mit

$$
x a \rightarrow x a f \beta=x f a \beta=x f .
$$

Eine Fortsetzung $f^{\prime}: A \rightarrow V$ liegt in $F_{1}\left[X a \in \mathcal{A}_{1}\left(f^{\prime}\right)\right]$.

Und auf $X a$ ist $f^{\prime} a=f\left[x a f^{\prime} a=x f a=x a f\right]$.

Es folgt $f^{\prime} a-f \in F_{0}$.

$\mathrm{Zu} w \in W$ existiert somit ein $w^{\prime} \in W$ mit $w^{\prime} a=w$, d.h. $a_{W}$ ist surjektiv.

Injektiv bedeutet $f a \notin F_{0}$ für $f \in F_{1} \backslash F_{0}$, folgt also aus (**).

3.6. Folgerung. Jeder Integritätsring hat einen Quotientenkörper. Genauer: Es gibt einen Monomorphismus in einen Körper.

Ein archimedischer Georing führt also zu einem archimedischen Geokörper, damit sogar zu einem vollständigen.

Beweis. Es gibt ein $V \neq 0$, nämlich $V=A$. Die Abbildung $a \rightarrow a_{W}$ ist ein Monomorphismus von $A$ in den Endomorphismenring $E$ von $W$, und in $E$ ist jedes $a_{W}(a \neq 0)$ invertierbar.

Bemerkung. Ist $U$ Teilmodul eines Moduls $W$ wie in 3.5, so bilden die Elemente $\frac{u}{a}=u\left(a_{W}\right)^{-1}$ einen Teilmodul $Q_{W}(U)$. Von dem Monomorphismus $\gamma$ in 3.5 kann also auch noch $W=\mathrm{Q}_{W}(V \gamma)$ verlangt werden. In der Tat haben $\gamma$ und $W$ im Beweis von 3.5 diese Eigenschaft.

Obiges Vorgehen geht auf den Hinweis eines Studenten zurück. Es ergibt sich zwanglos aus der Beobachtung, daß die Multiplikation mit $\frac{a}{b} \in \mathrm{Q}(A)$ ein $A$-Modulhomomorphismus von $A b$ in $A$ ist. 


\section{Geordnete Mengen, Gruppen und Ringe vom Typ $\mathbb{Z}$.}

4.1. Eine lineare Geomenge $V \neq \emptyset$ ist vom Typ $\mathbb{Z}$, wenn jede oben/unten beschränkte Teilmenge $\neq \emptyset$ ein Maximum/Minimum hat und $\neq V$ ist.

Gleichwertig: Jedes Element $a \in V$ hat einen Nachfolger $a^{+}=\min \left(V_{>a}\right)$ und einen Vorgänger $a^{-}=\max \left(V_{<a}\right)$, und

$(*) V$ ist die einzige Teilmenge $\neq \emptyset$, die mit $a$ auch $a^{+}$und $a^{-}$enthält.

Bemerkung. Eine Geogruppe vom Typ $\mathbb{Z}$ ist archimedisch.

4.2. SAtz. Sei $V$ eine Geomenge vom Typ $\mathbb{Z}$ mit Nachfolgerfunktion $\nu: x \rightarrow x^{+}$. Sei $A=\operatorname{Aut}(V)$ die Automorphismengruppe. Wähle $v \in V$.

(a) $A$ ist scharf transitiv auf $V$, d. h. $\alpha \rightarrow v \alpha$ ist bijektiv von $A$ auf $V$.

(b) Bezüglich $\alpha \leq \beta: \Longleftrightarrow v \alpha \leq v \beta$ ist $A$ eine Geogruppe vom Typ $\mathbb{Z}$ mit Nachfolgerfunktion $\alpha \rightarrow \alpha \nu$. Diese Ordnung ist unabhängig von $v$.

Beweis. (a) Offenbar ist $\nu$ ein Automorphismus von $V$, der mit allen Automorphismen vertauschbar ist, d. h. $\nu$ liegt im Zentrum $Z=Z(A)$ von $A$.

Wegen $4.1(*)$ ist $v Z=V$ und $\{x \in V \mid x \alpha=x\}=\emptyset$ für jedes $\alpha \neq 1$ in $A$. Aus $v \alpha=v \beta$ folgt also $\alpha=\beta$. Somit ist $A=Z$ (abelsch).

(b) $A$ ist zunächst eine Geomenge, und $\alpha \rightarrow v \alpha$ ist ein Isomorphismus. Es folgt $v \alpha \nu=(v \alpha)^{+}=v \alpha^{+}$, also $\alpha \nu=\alpha^{+}$. Für $\alpha, \beta, \gamma \in A$ folgt weiter

$$
\alpha \leq \beta \Leftrightarrow v \alpha \leq v \beta \Leftrightarrow v \alpha \gamma \leq v \beta \gamma \Leftrightarrow \alpha \gamma \leq \beta \gamma
$$

sowie, mit $w=v \gamma$,

$$
w \alpha \leq w \beta \Leftrightarrow v \gamma \alpha \leq v \gamma \beta \Leftrightarrow v \alpha \gamma \leq v \beta \gamma \Leftrightarrow v \alpha \leq v \beta .
$$

4.3. Lemma. Sei $V$ eine Geogruppe vom Typ $\mathbb{Z}, u=\min \left(V_{0}\right)$, und $E$ der Endomorphismenring der abelschen Gruppe $V$.

(a) Für jedes $x \in V$ ist $x+u$ der Nachfolger von $x$.

(b) Die Gruppe $V$ wird von $u$ erzeugt.

(c) Ist $\beta \in E$ und $u \beta>0$, so ist $v \beta>0$ für alle $v>0$ in $V$.

(d) Mit $\alpha \leq \beta: \Longleftrightarrow u \alpha \leq u \beta$ ist $E$ ein Georing vom Typ $\mathbb{Z}$.

(e) Sei $W$ eine weitere Geogruppe und $\varphi: V \rightarrow W$ ein Ordnungsisomorphismus mit $0 \varphi=0$. Dann ist $\varphi$ auch ein Gruppenisomorphismus.

Beweis. (a) folgt aus $x<x+u \leq x+\left(x^{+}-x\right)=x^{+}$, und (b) dann sofort aus 4.1(*): die von $u$ erzeugte Untergruppe ist $=V$.

(c) Sei $v$ ein Gegenbeispiel. Wähle $v$ minimal. Dann ist $0<v-u<v$, also $\operatorname{doch} v \beta=(u+(v-u)) \beta=u \beta+(v-u) \beta>0$. 
(d) Der von 1 erzeugte Teilring $F$ von $E$ liegt im Zentrum $Z(E)$.

Die Untergruppe $u F$ von $V$ enthält $u$ und ist daher gleich $V$ [4.3(b)]. Genauso folgt $u \notin \operatorname{Kern}(\gamma)$ für alle $\gamma \neq 0$ in $E$, d. h. $u \alpha \neq u \beta$ für $\alpha \neq \beta$.

Somit ist $E=F$ kommutativ und $\alpha \rightarrow u \alpha$ bijektiv. Laut (c) folgt $u \alpha \beta>0$ aus $u \alpha>0$ und $u \beta>0$, d. h. aus $\alpha>0$ und $\beta>0$ folgt $\alpha \beta>0$.

(e) Sei $X=\{x \in V \mid(v+x) \varphi=v \varphi+x \varphi$ für alle $v \in V\}$, also $0 \in X$.

Weiter ist $u \varphi=\min \left(W_{0}\right)$, also $w^{+}=w+u \varphi$ für alle $w \in W$.

Mit $v \in V$ und $w=v \varphi$ folgt $v^{ \pm} \varphi=w \pm u \varphi$, für alle $x \in X$ also

$$
\left(v+x^{ \pm}\right) \varphi=(v+x \pm u) \varphi=(v \pm u) \varphi+x \varphi=w \pm u \varphi+x \varphi=w+x^{ \pm} \varphi,
$$

und damit ist $X=V[4.1(*)]$.

4.4. Lemma. In einem Georing $V$ vom Typ $\mathbb{Z}$ ist $\min \left(V_{0}\right)=1$.

Ist $W$ ein weiterer Georing und $\varphi: V \rightarrow W$ ein Ordnungsisomorphismus mit $0 \varphi=0$, so ist $\varphi$ auch ein Ringisomorphismus.

Beweis. Wäre $u=\min \left(V_{0}\right)<1$, so wäre $0<u u<u$. Also ist $u=1$, genauso $\min \left(W_{0}\right)=1$ und folglich $1 \varphi=1$. Schon wegen $0 \varphi=0$ ist

$$
X:=\{x \in V \mid(v x) \varphi=v \varphi \cdot x \varphi \text { für alle } v \in V\}
$$

nicht leer, und da $\varphi$ additiv ein Isomorphismus ist [4.3(e)], folgt weiter, daß $X$ mit $x$ auch $x^{ \pm}=x \pm 1$ enthält, also $=V$ ist $[4.1(*)]$ :

$$
(v(x \pm 1)) \varphi=(v x \pm v) \varphi=(v x) \varphi \pm v \varphi=v \varphi \cdot x \varphi \pm v \varphi=v \varphi \cdot(x \pm 1) \varphi,
$$

4.5. Lemma [Beweis in 6.7]. (a) Zu Geomengen $V, V^{\prime}$ vom Typ $\mathbb{Z}, a \in V$, $a^{\prime} \in V^{\prime}$ gibt es genau einen Isomorphismus $V \rightarrow V^{\prime}$ mit $a \rightarrow a^{\prime}$.

(b) Sei $V$ eine Menge und $\nu: V \rightarrow V$ bijektiv mit $A \nu \subset A$ für eine Teilmenge $A$, aber $X \nu=X$ für keine Teilmenge $X \neq \emptyset, V$.

Bezüglich einer gewissen Ordnung, der einzigen mit $x<x \nu$, ist $V$ vom Typ $\mathbb{Z}$ mit Nachfolgerfunktion $\nu$.

4.6. Folgerung. (a) $\mathrm{Zu}$ geordneten Gruppen oder Ringen $V, W$ vom Typ $\mathbb{Z}$ existiert genau ein Isomorphismus $V \rightarrow W$.

(b) Sei $G$ eine Geogruppe und $0<u \in G$. Dann ist die von $u$ erzeugte Untergruppe vom Typ $\mathbb{Z}$ mit $x^{+}=x+u$.

4.7. In jedem Ring $K$ ist die von 1 erzeugte additive Untergruppe $V$ ein Teilring, denn $\{x \in K \mid V x \subseteq V\}$ ist ein Teilring $\subseteq V$, also $=V$.

In einem Georing ist dieser Primring also vom Typ $\mathbb{Z}$.

$\mathrm{Zu}$ vollständigen Geokörpern $L, L^{\prime}$ gibt es daher genau einen Isomorphismus zwischen $L$ und $L^{\prime}[2.8,2.9,3.2]$. 


\section{Wohlgeordnete Mengen und der $f$-Kettensatz.}

5.1. Unter einem Abschnitt einer Geomenge $G$ ist jetzt immer ein unterer Abschnitt zu verstehen, und $G_{a}$ steht demgemäß für $G_{<a}=\{x \in G \mid x<a\}$.

$\mathcal{A}(G)$ ist die Menge aller Abschnitte $A$ von $G$, und $\mathcal{A}^{*}(G)$ die aller echten Abschnitte $(A \neq G)$. Das Symbol $G \mid X$ reduziert auf Abschnitte $A \supseteq X$.

Eine lineare Geomenge $W$ heißt wohlgeordnet, auch Kette, wenn jede nichtleere Teilmenge ein Minimum hat. Auch jede Teilmenge ist dann eine Kette.

Die Ketten einer Geomenge sind die wohlgeordneten Teilmengen.

Eine Folge ist eine auf einer Kette definierte Abbildung.

\subsection{In einer Kette $W$ hat jedes $a \notin \operatorname{Max}(W)$ einen Nachfolger $a^{+}$.}

Die Abbildung $x \rightarrow W_{x}$ ist ein Isomorphismus von $W$ auf die [durch $\subseteq$ ] geordnete Menge $\mathcal{A}^{*}(W)$. Insbesondere ist auch $\mathcal{A}(W)$ wohlgeordnet.

Das Urbild $x$ von $A \in \mathcal{A}^{*}(W)$ ist das Folgeelement

$$
f^{+}(A)=\min (W \backslash A)=\max \left(A^{+}\right)
$$

von $A$, und $A \cup\{x\}$ ist der Nachfolger $A^{+}$von $A$ in $\mathcal{A}(W)$.

Genau dann hat $B \in \mathcal{A}(W)$ einen Vorgänger, wenn $B$ ein Maximum hat. Und genau dann hat $B$ keinen Vorgänger, wenn $B$ die Vereinigung der echten Abschnitte von $B$ ist.

Nenne $W$ grundiert, wenn jedes $x \in W \backslash \operatorname{Min}(W)$ einen Vorgänger hat. Gleichwertige Eigenschaften:

(a) Jeder nichtleere echte Abschnitt hat einen Vorgänger.

(b) Jeder nichtleere echte Abschitt hat ein Maximum.

(c) Jede nichtleere beschränkte Teilmenge hat ein Maximum.

(d) Jede unter $x \rightarrow x^{+}$abgeschlossene Teilmenge ist ein oberer Abschnitt von $W$ (ist also $=W$ wenn $\operatorname{sie} \operatorname{Min}(W)$ enthält).

Jede Teilmenge eines grundierten $W$ ist grundiert, wie auch jede unten beschränkte Teilmenge einer Geomenge vom Typ $\mathbb{Z}$.

Ein grundiertes nichtleeres $W$ ohne Maximum ist vom Typ $I N$.

5.3. Induktionslemma. Sei $W$ eine Kette und $\mathcal{A} \subseteq \mathcal{A}(W)$ mit $A^{+} \in \mathcal{A}$ und $\bigcup_{\mathcal{B}} \in \mathcal{A}$ für $A \neq W$ in $\mathcal{A}$ und $\emptyset \neq \mathcal{B} \subseteq \mathcal{A}$.

Dann ist $\mathcal{A}$ ein oberer Abschnitt von $\mathcal{A}(W)$.

Also: Eine nichtleere Eigenschaft von Abschnitten, die sich auf Nachfolger und nichtleere Vereinigungen vererbt, gilt auch für $W$.

Beweis. Nehme $A \in \mathcal{A}$ und $B \in \mathcal{A}(W) \backslash \mathcal{A}$ mit $A \subset B$ an. Wähle $B$ minimal (bei festem $A$ ). Jedes $X \in \mathcal{A}^{*}(B \mid A)$ liegt in $\mathcal{A}$. Also hat $B$ keinen Vorgänger und ist auch nicht die Vereinigung dieser $X$, ein Widerspruch. 
5.4. Folgerung. (a) Sei $W$ eine Kette und $\leq^{\prime}$ eine weitere Ordnung auf (der Menge) $W$. Für jedes $A \in \mathcal{A}^{*}(W)$ mit $\leq=\leq^{\prime}$ auf $A$ sei $x<^{\prime} f^{+}(A)$ für alle $x \in A$. Dann ist $\leq=\leq^{\prime}$.

(b) Für grundiertes $W$ genügt $x<^{\prime} x^{+}$für alle $x \in W \backslash \operatorname{Max}(W)$, und dasselbe gilt für Geomengen $W$ vom Typ $\mathbb{Z}$ (denn $W_{\geq x}$ ist vom Typ $\mathbb{I N}$ ).

5.5. Der $f$-Kettensatz (nach H. Kneser [14]).

In der allgemeinen Version wird eine Menge $M$ und eine Teilmenge $\mathcal{A}$ von $\mathcal{P}(M)$ vorausgesetzt, ferner $f: \mathcal{A} \rightarrow M$ und $f(A) \notin A$ für alle $A \in \mathcal{A}$.

Sodann wird $W \in \mathcal{P}(M)$ eine $f$-Kette genannt, wenn es eine Wohlordnung auf $W$ gibt mit $\mathcal{A}^{*}(W) \subseteq \mathcal{A}$ und $f^{+}(A)=f(A)$ für alle $A \in \mathcal{A}^{*}(W)$.

In der Version für eine geordnete Menge $M$ ist immer $x<f(A)$ für alle $x \in A$, und die Ordnung einer $f$-Kette ist die durch $M$ gegebene.

Die Vereinigung $V$ aller $f$-Ketten ist jeweils eine $f$-Kette mit $V \notin \mathcal{A}$.

Beweis. (1) Sind $W$ und $W^{\prime} f$-Ketten (bezüglich $\leq$ und $\leq^{\prime}$ ), so ist $W \in \mathcal{A}\left(W^{\prime}\right)$ oder $W^{\prime} \in \mathcal{A}(W)$ (und $\leq=\leq^{\prime}$ auf $W$ bzw. $W^{\prime}$ ).

Denn mit der Vereinigung $U$ aller gemeinsamen Abschnitte $A$ (mit $\leq=\leq^{\prime}$ auf $A$ ) wäre im Falle $W \neq U \neq W^{\prime}$ auch $U \cup\{f(U)\}$ ein solcher Abschnitt.

(2) $\mathrm{Zu} a, b, c \in V$ gibt es eine $f$-Kette $W$ mit $a, b, c \in W$.

(3) Es gibt genau eine Relation $\leq$ auf $V$, die die "f-Ordnung“ einer jeden $f$-Kette fortsetzt. Sie ist eine lineare Ordnungsrelation.

(4) Jede $f$-Kette $A$ ist ein Abschnitt von $V$, und für $X \subseteq V$ folgt daher $\operatorname{Min}(A \cap X) \subseteq \operatorname{Min}(X)$, also $\operatorname{Min}(X) \neq \emptyset$ falls $A \cap X \neq \emptyset$.

Somit ist $V$ wohlgeordnet durch $\leq$, und sogar eine $f$-Kette:

Für $A \in \mathcal{A}^{*}(V), b=f_{V}^{+}(A)$ und eine $f$-Kette $B$ mit $b \in B$ folgt $A \in \mathcal{A}^{*}(B)$ und $b=f_{B}^{+}(A)=f(A)$.

Im Falle $V \in \mathcal{A}$ wäre $V \cup\{f(V)\}$ eine $f$-Kette.

Bemerkung. Bei den folgenden Hauptanwendungen des $f$-Kettensatzes erfordert die Existenz von $f$ das Auswahlaxiom.

5.6. Das Lemma von Zorn. Hat jede Kette in einer Geomenge $G$ eine obere Schranke, so hat $G$ ein maximales Element.

5.7. Der Wohlordnungssatz von Zermelo.

Jede Menge besitzt eine Wohlordnung.

$\mathrm{Zu}$ 5.6: Andernfalls hat jede Kette $A$ sogar eine obere Schranke $f(A) \notin A$.

$\mathrm{Zu}$ 5.7: In 5.5 ist $V=M$ wenn $\mathcal{A}=\mathcal{P}(M) \backslash\{M\}$. 
5.8. Sei $M$ eine Menge, $\alpha: M \rightarrow M$ und $X \subseteq M$.

Das $\alpha$-Erzeugnis $\langle X\rangle_{\alpha}$ von $X$ ist die kleinste $\alpha$-invariante Teilmenge $\supseteq X$ von $M$, d. h. der Schnitt aller Teilmengen $Y \supseteq X, Y \alpha$.

Grundregeln. (a) $\langle X\rangle_{\alpha}=X \cup\langle X\rangle_{\alpha} \alpha=X \cup\langle X \alpha\rangle_{\alpha}$.

(b) $\langle X\rangle_{\alpha} \beta=\langle X \beta\rangle_{\alpha}$ für jedes $\beta: M \rightarrow M$ mit $\alpha \beta=\beta \alpha$.

Beweis. (a) $\ldots \cup \ldots$ liegt in $\langle X\rangle_{\alpha}$, enthält $X$ und ist $\alpha$-invariant.

(b) $\supseteq:\langle X\rangle_{\alpha} \beta$ enthält $X \beta$ und ist $\alpha$-invariant.

$\subseteq$ : Mit $U=\langle X \beta\rangle_{\alpha}$ ist auch das Urbild $U \beta^{-1}(\supseteq X) \alpha$-invariant und enthält daher $\langle X\rangle_{\alpha}$. Es folgt $\langle X\rangle_{\alpha} \beta \subseteq U \beta^{-1} \beta \subseteq U$.

\subsection{Der erste $I N$-Satz von Dedekind.}

Sei $M$ eine Menge, $\nu: M \rightarrow M$ injektiv, $u \in M \backslash M \nu$ und $N=\langle u\rangle_{\nu}$.

Bezüglich einer gewissen Ordnung ist $N$ dann vom Typ $I N$ mit Nachfolgerfunktion $\nu$ und Minimum $u$.

Beweis. Sei $\mathcal{A}$ die Menge aller $A \subseteq N$ mit $A=\emptyset$ oder $a \nu \notin A$ für genau ein $a \in A$. Setze $f(\emptyset)=u$ bzw. $f(A)=a \nu$. Wende 5.5 an. Erhalte $V$.

Zunächst ist $u=f^{+}(\emptyset)=\min (V)$, denn $\{u\}$ ist eine $f$-Kette.

Für $\emptyset \neq A \in \mathcal{A}^{*}(V)$ ist $f^{+}(A)=f(A)=a \nu$, also $a=\max (A)$.

Somit ist $V$ grundiert und $x^{+}=x \nu$ für alle $x \in V \backslash \operatorname{Max}(V)$, weiter $\operatorname{Max}(V)=\emptyset$ wegen $V \notin \mathcal{A}$, also $V \nu \subseteq V$ und daher $V=N$.

5.10. Sei $W$ vom Typ $I N$ mit Minimum $u$ und Nachfolgerfunktion $\nu$.

(a) Mit $\alpha=\nu^{2}, U=\langle u\rangle_{\alpha}$ und $V=U \nu$ gilt $W=U \dot{\cup} V$.

(b) Bezüglich einer neuen Ordnungsrelation $\leq$ ' ist $W$ vom Typ $\mathbb{Z}$, sie ist $\leq$ auf $U, \geq$ auf $V$, und $x \leq^{\prime} y$ gilt noch für $x \in V, y \in U$.

Anschaulich: $0,1,2,3, \ldots$ wird $\mathrm{zu} \quad \ldots 5,3,1,0,2,4, \ldots$ umgeordnet.

Beweis. Wegen $V \nu=U \alpha \subseteq U$ ist $U \cup V \nu$-invariant, also $=W[5.2(\mathrm{~d})]$.

Sei $U \cap V \neq \emptyset$. Dann liegt $m=\min (U \cap V)$ nicht in $(U \cap V) \alpha=U \alpha \cap V \alpha$ [ $\alpha$ ist injektiv], wegen $U=\{u\} \cup U \alpha$ und $V=\{u \nu\} \cup V \alpha$ aber doch.

$\mathrm{Zu}$ (b) ist nur zu beachten, daß $U$ und $V$ vom Typ $I N$ sind.

5.11. Der $f$-Kettensatz führt auch zur Existenz einer maximalen Kette, einer Kompositionsreihe, in einer Geomenge, wenn jede oben echt beschränkte Kette einen oberen Nachbarn hat, eine minimale echte obere Schranke.

Beispiel: Der Teilmodulverband - die bezüglich $\subseteq$ geordnete Menge aller Teilmoduln - eines halbeinfachen Moduls.

Ein solcher ist die Summe der einfachen, d.h. minimalen Teilmoduln, und Standardbeispiel hierfür ist jeder Vektorraum.

Übrigens ist eine maximale Kette auch eine maximale lineare Teilmenge. 
5.12. Wenn schon Kompositionsreihen, dann auch Satz von Jordan-Hölder. Er betrifft Gruppen, auch mit Operatorenbereich. Für das wesentliche genügt der abelsche Fall. Seien $\mathcal{W}$ und $\mathcal{W}^{\prime}$ also Kompositionsreihen eines Moduls $M$, genauer des Teilmodulverbands $\mathcal{V}$.

Dann ist eine bijektive Abbildung $X \rightarrow X^{\prime}$ von $\mathcal{W}$ auf $\mathcal{W}^{\prime}$ aufzuspüren mit

$$
M^{\prime}=M \quad \text { und } \quad X^{+} / X \simeq X^{\prime+} / X^{\prime} \text { für alle } X \neq M .
$$

Betrachte zu gegebenem $X \in \mathcal{W}$ den aus allen $Y \in \mathcal{W}^{\prime}$ mit

(1) $Y \cap X^{+} \subseteq X$

bestehenden Abschnitt von $\mathcal{W}^{\prime}$. Die Vereinigung $X^{\prime}$ aller $Y$ liegt in $\mathcal{W}^{\prime}$, denn $\mathcal{W}^{\prime}$ ist auch eine maximale lineare Teilmenge von $\mathcal{V}$. Somit ist $X^{\prime}$ selbst ein $Y$, ist also das maximale $Y$. Schreibe daher $Y$ für $X^{\prime}$. Es folgt $Y \neq M$ und

(2) $Y^{+} \cap X^{+} \nsubseteq X$, also

(3) $X^{+}=X+D$ mit $D=Y^{+} \cap X^{+}$, und damit

(4) $X^{+} / X \simeq D / D \cap X$.

Behauptung: Das analog definierte $Y^{\prime} \in \mathcal{W}$ ist $=X$. Das bedeutet

$$
\text { (a) } X \cap Y^{+} \subseteq Y \text { und (b) } X^{+} \cap Y^{+} \nsubseteq Y \text {. }
$$

$\mathrm{Zu}$ (a): Sonst wäre $Y^{+}=Y+\left(X \cap Y^{+}\right)$, wegen $Y^{+} \supseteq D \supseteq X \cap Y^{+}$also $D=(Y \cap D)+\left(X \cap Y^{+}\right)$[Dedekinscher Modulsatz], folglich $D \subseteq X$ wegen $Y \cap D=Y \cap X^{+} \subseteq X[(1)]$, im Widerspruch zu (3).

Der Dedekindsche Modulsatz ist eine gruppentheoretische Banalität:

Für $A, B, C \in \mathcal{V}$ mit $A+B \supseteq C \supseteq B$ ist $C=(A \cap C)+B$.

$\mathrm{Zu}$ (b): Sonst wäre $D=X^{+} \cap Y \subseteq X$.

Damit ist die Bijektivität erledigt, und die angekündigte Isomorphie folgt aus $D \cap X=X \cap Y=D \cap Y$, denn analog (4) ist $Y^{+} / Y \simeq D / D \cap Y$.

Es folgt eine schöne Anwendung.

Unser Modul $M$ sei direkte Summe gewisser einfacher Teilmoduln, sagen wir $X \in \mathcal{B}$, wie etwa bei $\mathcal{B}=\{K x \mid x \in B\}, B$ Basis eines Vektorraums über dem Divisionsring $K$.

Die Teilmenge $\mathcal{B}$ von $\mathcal{V}$ besitzt eine Wohlordnung $\leq$, und die Teilmoduln $\mathcal{W}_{X}(X \in \mathcal{B})$, definiert als Summe aller $Y<X$ in $\mathcal{B}$, bilden dann zusammen mit $M$ eine Kompositionsreihe $\mathcal{W}$ mit $|\mathcal{W} \backslash\{M\}|=|\mathcal{B}|$.

Somit sind all diese $\mathcal{B}$ 's gleichmächtig, und insbesondere sind alle Basen eines Vektorraums gleichmächtig.

Eine schöne Anwendung des vollen Satzes von Jordan-Hölder ist ein gewisser Zugang zur Determinantenfunktion [math.uni-kiel.de/algebra/bender]. 


\section{Induktion und Kardinalität.}

$W$ und $W^{\prime}$ seien Ketten. Nenne $\alpha: W \rightarrow W^{\prime}$ einen Morphismus und schreibe $\alpha: W \nearrow W^{\prime}$, wenn aus $x<y(x, y \in W)$ immer $x \alpha<y \alpha$ folgt und $W \alpha$ ein Abschnitt von $W^{\prime}$ ist; kurz: $\alpha$ ist ein Isomorphismus von $W$ auf einen Abschnitt von $W^{\prime}$. Schreibe $W \nearrow W^{\prime}$ wenn ein $\alpha$ existiert.

6.1. Hilfssatz. Die Menge $V$ sei Vereinigung von gewissen Teilmengen, sagen wir $A \in \mathcal{B}$, derart daß $A \subseteq B$ oder $B \subseteq A$ für alle $A, B \in \mathcal{B}$.

Zu jedem $A$ sei eine Abbildung $\alpha_{A}$ von $A$ in eine weitere Menge $M$ gegeben, und im Falle $A \subseteq B$ sei $\alpha_{A}=\alpha_{B}$ auf $A$.

Dann existiert genau eine Fortsetzung $\alpha: V \rightarrow M$ aller $\alpha_{A}$.

\subsection{Der Satz Über die induktive Definition von Folgen.}

Sei $A_{0} \in \mathcal{A}(W)$ und $M$ eine Menge. Setze $F_{A}=\mathcal{F}(A, M)$ für $A \in \mathcal{A}\left(W \mid A_{0}\right)$. Für $\alpha \in F_{A}(A \neq W)$ sei immer eine Fortsetzung $\alpha^{+} \in F_{A^{+}}$gegeben.

Dann hat ein $\alpha_{0}: A_{0} \rightarrow M$ genau eine Fortsetzung $\alpha \in F_{W}$ mit

$$
\left(\alpha_{\mid A}\right)^{+}=\alpha_{\mid A^{+}} \text {für alle } A \neq W .
$$

Bemerkung. Das analog zu $X \in \mathcal{A}\left(W \mid A_{0}\right)$ gehörige $\alpha_{X}$ ist $=\alpha_{\mid X}$.

Beweis. Nehme $W$ als minimales Gegenbeispiel in $\mathcal{A}\left(W \mid A_{0}\right)$ an.

Erhalte $\alpha_{U}: U \rightarrow M$ analog $\alpha$ für jedes $U \in \mathcal{B}=\mathcal{A}^{*}\left(W \mid A_{0}\right)$.

Die Vereinigung $V=\bigcup_{\mathcal{B}}$ aller $U$ ist ein Abschnitt $\supseteq A_{0}$ von $W$, und im Falle $V \in \mathcal{B}$ ist $V^{+}=W$ und $\alpha=\left(\alpha_{V}\right)^{+}$wie gewünscht.

Daher ist $V=W$, und dann ist das aus 6.1 resultierende $\alpha: V \rightarrow M$ als Fortsetzung aller $\alpha_{U}$ wie gewünscht.

6.3. Folgerung. Sei $m_{0} \in M$ und $W$ grundiert mit Minimum $w_{0}$, weiter jedem $w \in W \backslash \operatorname{Max}(W)$ eine Abbildung $f_{w}: M \rightarrow M$ zugeordnet.

Dann existiert genau eine Abbildung $\alpha: W \rightarrow M$ mit $w_{0} \alpha=m_{0}$ und $w^{+} \alpha=f_{w}(w \alpha)$ für alle $w$.

Beispiel. Sei $M$ eine Halbgruppe und $h: W \rightarrow M$. Dann existiert genau ein $\alpha: W \rightarrow M$ mit $w_{0} \alpha=h\left(w_{0}\right)$ und $w^{+} \alpha=w \alpha \cdot h\left(w^{+}\right)$.

Hier ist $f_{w}(x)=x \cdot h\left(w^{+}\right)$, und $w \alpha(w \in W)$ ist anschaulich das Produkt der Elemente $h(u)(u \leq w)$ in der durch $W$ gegebenen Reihenfolge.

6.4. Cantors Isomorphiesatz für Wohlgeordnete Mengen.

(a) Es gibt höchstens ein $\alpha: W \nearrow W^{\prime}$.

(b) Es gilt $W \nearrow W^{\prime}$ oder $W^{\prime} \nearrow W$.

(c) Gilt beides in (b), so sind $W$ und $W^{\prime}$ isomorph $\left(W \simeq W^{\prime}\right)$.

Bemerkung. Aus (a) ergibt sich sofort die wichtige Eigenschaft einer wohlgeordneten Menge, nicht zu einem echten Abschnitt isomorph zu sein. 
Beweis. (a) Sei $\beta$ wie $\alpha$. Wende 5.3 auf $\mathcal{A}=\left\{A \in \mathcal{A}(W) \mid \alpha_{\mid A}=\beta_{\mid A}\right\}$ an.

(b) Beides sei falsch. Für $A \in \mathcal{A}(W)$ und $\alpha: A \nearrow W^{\prime}$ ist dann $A \neq W$ und $A \alpha \neq W^{\prime}$, und daraus resultiert eine Fortsetzung $\alpha^{+}: A^{+} \nearrow W$; sie bildet das Folgeelement von $A$ in $W$ auf das Folgeelement von $A \alpha$ in $W^{\prime}$ ab.

Nach (a) ist $\alpha=\beta$ auf $A$ wenn $A \subseteq B \in \mathcal{A}(W)$ und $\beta: B \nearrow W^{\prime}$.

Sei $W$ sogar ein minimales Gegenbeispiel in $\mathcal{A}(\mathcal{W})$, bei festem $W^{\prime}$.

Zu jedem $U \in \mathcal{B}=\mathcal{A}^{*}(W)$ existiert dann $\alpha_{U}: U \nearrow W^{\prime}$.

Mit $U$ liegt also auch $U^{+}$in $\mathcal{B}$, und das bedeutet $W=\cup_{\mathcal{B}}$. Das aus 6.1 resultierende $\alpha: W \rightarrow W^{\prime}$ ist als Fortsetzung aller $\alpha_{U}$ offenbar ein Morphismus.

(c) Sei $\alpha: W \nearrow W^{\prime}$ und $\alpha^{\prime}: W^{\prime} \nearrow W$. Dann ist $\alpha \alpha^{\prime}: W \rightarrow W$ ein Morphismus, also $=\operatorname{id}_{W}$ wegen (a), und damit ist auch $\alpha^{\prime} \alpha=\mathrm{id}_{W^{\prime}}$.

6.5. Folgerung [aus 6.4 und 5.7].

(a) Sind $A$ und $B$ beliebige Mengen, so ist $|A| \leq|B|$ oder $|B| \leq|A|$, d. h. es gibt eine injektive Abbildung von $A$ in $B$ oder von $B$ in $A$.

(b) Eine nichtleere Menge $\mathcal{A}$ von Mengen hat bezüglich $|\ldots| \leq|\ldots|$ ein Minimum $A(|A| \leq|B|$ für alle $B \in \mathcal{A})$.

\subsection{Der Satz von Schröder-Bernstein.}

Aus $|A| \leq|B|$ und $|B| \leq|A|$ folgt $|A|=|B|$, d. h. es gibt eine bijektive Abbildung zwischen $A$ und $B$.

Beweis (Dedekind). Sei $\alpha: A \rightarrow B$ injektiv. Darf $B \subseteq A$ annehmen und muß ein bijektives $\gamma: B \rightarrow A \alpha$ finden. Nun ist $\alpha: A \rightarrow A$ und $B \alpha \subseteq B$.

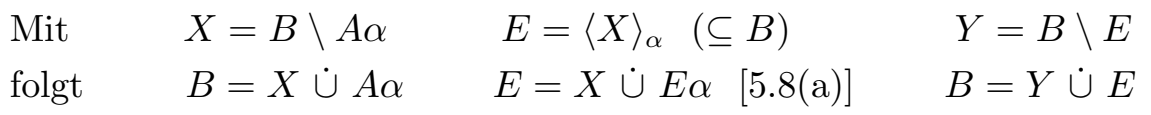
und damit auch noch $Y=B \backslash E=A \alpha \backslash E \alpha$, d.h. $\quad A \alpha=Y \dot{\cup} E \alpha$.

Definiere nun $\gamma$ auf $B=Y \dot{\cup} E$ als $\operatorname{id}_{Y}$ auf $Y$ und als $\alpha$ auf $E$.

6.7. Sind $W$ und $W^{\prime}$ vom Typ $I N$, so existiert genau ein Isomorphismus $\alpha: W \rightarrow W^{\prime}$, aufgrund 6.4(a)(b) und 5.2(b).

Daraus folgt sofort 4.5(a), denn dort sind $V_{>a}$ und $V_{>a^{\prime}}^{\prime}$ vom Typ $I N$, und $V_{<a}$ ist wie $V_{<a^{\prime}}^{\prime}$ dual vom Typ $I N$.

Beweis von 4.5(b): Wende 5.9(a) auf $N=\langle u\rangle_{\nu}$ mit $u \in A \backslash A \nu$ an: Bezüglich einer Ordnung $\leq$ ist $N$ vom Typ $I N$ mit Minimum $u$ und $x^{+}=x \nu$.

Analog: $\nu^{\prime}=\nu^{-1}, A^{\prime}=V \backslash A, u^{\prime}=u \nu^{\prime}, N^{\prime}$ und $\leq^{\prime}$.

Weiter ist $N \cap N^{\prime} \subseteq A \cap A^{\prime}=\emptyset$ und - wegen $N=N \nu \cup\{u\}$ und $N^{\prime}=$ $N^{\prime} \nu^{\prime} \cup\left\{u^{\prime}\right\}-\operatorname{auch}\left(N \cup N^{\prime}\right) \nu=N \cup N^{\prime}$, also $N \cup N^{\prime}=V$.

Erweitere nun $\leq$ zu einer Relation auf $V: x \leq y$ gilt noch für $x \in N^{\prime}$ und $y \in N$, sowie für $x, y \in N^{\prime}$ mit $y \leq^{\prime} x$. Zur Einzigkeit siehe 5.4(b). 


\section{Endliche und unendliche Mengen.}

7.1. Eine Menge $M$ ist endlich, wenn jedes injektive $\alpha: M \rightarrow M$ auch surjektiv ist.

Unendlichkeit läuft also auf die Existenz einer injektiven, aber nicht surjektiven Abbildung hinaus. Das Hauptbeispiel ist die Nachfolgerfunktion $x \rightarrow x^{+}$ einer nichtleeren Kette ohne Maximum. Umgekehrt impliziert die Existenz einer unendlichen Menge auch die Existenz geordneter Mengen vom Typ IN und $\mathbb{Z}[5.9,5.10(\mathrm{~b})]$, und über Gruppen und Ringe vom Typ $\mathbb{Z}[4.2(\mathrm{~b}), 4.3(\mathrm{~d})]$ ergeben sich dann vollständig geordnete Körper.

Offenbar ist die leere Menge endlich, auch jede Teilmenge und jedes bijektive Bild einer endlichen Menge, also auch jedes surjektive Bild [ordne jedem Bildelement ein Urbild zu, mit Hilfe des Auswahlaxioms].

$W$ ist wieder eine Kette vom Typ $I N$.

7.2. Hilfssatz. Sei $M=A \cup\{b\}$ eine Menge, $A$ endlich.

Dann ist auch $M$ endlich.

Beweis. Sei $\alpha: M \rightarrow M$ injektiv, aber nicht surjektiv.

Dann ist $A \alpha \nsubseteq A$, denn sonst wäre $A \alpha=A$, also $b \alpha=b$.

Sei also $a \in A$ und $a \alpha=b$, weiter $\tau$ die Transposition von $M$ mit $a \tau=b$ (und $b \tau=a$ ). Dann ist $\alpha^{\prime}=\alpha \tau$ wie $\alpha$, im Widerspruch zu $A \alpha^{\prime} \subseteq A$.

7.3. Lemma. (a) Die echten Abschnitte von $W$ sind endlich, und eine endliche Kette ist zu genau einem solchen Abschnitt isomorph.

(b) Ketten vom Typ $I N$ sind isomorph (schon bekannt), und eine unendliche Kette hat genau einen Abschnitt vom Typ $I N$.

Beweis. Der kleinste unendliche Abschnitt von $W$ hat nach 7.2 keinen Vorgänger, ist also $=W$. Alles weitere folgt aus dem Isomorphiesatz [6.4].

\subsection{DeR ZWeite $I N$-SAtZ von Dedekind.}

(a) Eine endliche Menge $M$ ist zu genau einem echten Abschnitt $A$ von $W$ gleichmächtig (man kann also $|M|=f^{+}(A)$ definieren).

(b) Genau dann ist eine Menge $M$ unendlich, wenn es eine injektive Abbildung von $W$ in $M$ gibt.

Beweis. (a) Da $M$ eine Wohlordnung besitzt [5.7], existiert nach 7.3(a) jedenfalls eine bijektive Abbildung von $M$ auf ein $A \in \mathcal{A}^{*}(W)$.

Ist $B$ wie $A$, mit $A \subseteq B$, so gibt es eine bijektive Abbildung von $B$ auf $A$, und daraus folgt $A=B$, denn $B$ ist endlich.

(b) Ist $\alpha: W \rightarrow M$ injektiv, so ist mit $W$ auch $W \alpha$ unendlich und damit auch $M$. Umgekehrt folgt die Existenz von $\alpha$ aus 7.3(b), wieder über 5.7. 


\section{Endliche Summen und Produkte.}

8.1 Sei $W$ eine endliche Kette, $H$ eine Halbgruppe und $h: W \rightarrow H$.

Durch 6.3 (Beispiel) wird jedem $A \in \mathcal{A}(W)$ ein Element $\pi(A) \in H$ zugeordnet, mit $\pi(\emptyset)=1$ und $\pi\left(A^{+}\right)=\pi(A) h\left(f^{+}(A)\right)$ für alle $A \neq W$.

Da es nur eine solche Abbildung $\pi: \mathcal{A}(W) \rightarrow H$ gibt, ist $\pi$ auf $\mathcal{A}(U)$ gleich dem analog zu $U \in \mathcal{A}(W)$ gehörigen $\pi_{U}$.

Da auch jede Teilmenge $B$ von $W$ eine endliche Kette ist, ist auch $\pi(B) \in H$ definiert (bezüglich $h_{\mid B}$ ), und für $B=W \backslash A(A \in \mathcal{A}(W))$ folgt per Induktion nach $W$ noch $\pi(W)=\pi(A) \pi(B)$, denn mit $w=\max (W), W_{1}=W \backslash\{w\}$ und $B_{1}=B \backslash\{w\}$ gilt (für $A \subset W$ )

$$
\pi(W)=\pi\left(W_{1}\right) h(w)=\pi(A) \pi\left(B_{1}\right) h(w)=\pi(A) \pi(B)
$$

unter der Annahme, daß die Behauptung für $W_{1}$ anstelle $W$ richtig ist.

Und mehr ist zum Thema geordnete Produkte nicht zu sagen.

8.2. Sei also $W$ jetzt irgendeine endliche Menge und $H$ kommutativ.

Der übliche Weg zum Produkt der Elemente $h_{x}=h(x)(x \in W)$ führt über eine Wohlordnung von $W$ und den Nachweis, daß das durch 8.1 gegebene Produkt unabhängig ist von der ausgewählten Wohlordung. Das Ergebnis läßt sich dann so formulieren:

SATZ. Es gibt genau ein $\pi: \mathcal{P}(W) \rightarrow H$ mit $\pi(\emptyset)=1, \pi(\{x\})=h_{x}$ für $x \in W$, und $\pi(X \cup Y)=\pi(X) \pi(Y)$ für disjunkte $X, Y \subseteq W$.

Bemerkung. Das analog zu einer Teilmenge $U \subseteq W$ (und $h_{\mid U}$ ) gehörige $\pi_{U}$ ist $=\pi$ auf $\mathcal{P}(U)$ [wegen der Einzigkeit von $\left.\pi_{U}\right]$.

Außerdem ist $\pi(W)=\pi\left(W_{1}\right) h_{w}$, für $w \in W$ und $W_{1}=W \backslash\{w\}$.

Es folgt ein direkter Beweis für den Satz:

Nehme $W \neq \emptyset$ an. Per Induktion ist die Behauptung für jedes $V \subset W$ richtig $[6.5(\mathrm{~b})]$. Damit liegt $\pi(V)$ für diese $V$ 's vor, und mit $\pi(W)=\pi\left(W_{1}\right) h_{w}$ $\left(w, W_{1}\right.$ wie oben $)$ ist dann $\pi: \mathcal{P}(W) \rightarrow H$ definiert.

Beim Nachweis der Produktregel ist OBdA $w \in Y \subset W=X \cup Y$, somit

$$
\pi(W)=\pi\left(W_{1}\right) h_{w}=\pi(X) \pi\left(Y \cap W_{1}\right) h_{w}=\pi(X) \pi(Y) .
$$

8.3. Unter „Induktion" verstehe ich offenbar nicht den herkömmlichen Schluß von $n$ auf $n+1$, sondern das Beweisen unter der Gratis-Zusatzbedingung, daß die im jeweiligen Sinne kleineren Objekte die fragliche Eigenschaft haben. Immer ist jedem der betrachteten Objekte ein Element einer artinsch geordneten Menge $M$ zugeordnet. Artinsch bedeutet, daß jede nichtleere Teilmenge ein minimales Element hat. Gewöhnlich ist $M$ sogar wohlgeordnet oder eine Menge endlicher Mengen (nach 6.5(b) artinsch bezüglich $\subseteq$ ). 
8.4. Bei additiver Notation steht $\Sigma_{x \in X} h_{x}$ oder kurz $\Sigma_{X}$ für $\pi(X)$.

Die Regeln (1) und (2) ergeben sich mittels Induktion nach $W$ aus den Grundregeln in 8.2. In (1) ist $P$ eine Partition von $W$, und in (2) ist $y$ grundsätzlich ein Endomorphismus der Halbgruppe $H$, allgemeiner ein Homomorphismus in eine weitere Halbgruppe. Man denke aber vor allem an die Elemente eines Ringes mit additiver Gruppe $H$.

(1) $\Sigma_{W}=\Sigma_{X \in P} \Sigma_{X}$,

(2) $\quad\left(\Sigma_{x \in X} h_{x}\right) y=\Sigma_{x \in X} h_{x} y$.

Es folgt das vertraute Ausmultipliziere eines Produktes von Summen:

(3) $\Sigma_{x \in X} h_{x} \cdot \Sigma_{y \in Y} k_{y}=\Sigma_{x \in X, y \in Y} h_{x} k_{y}$.

Unter Beachtung von (2) wird dafür (1) auf die aus den Teilmengen

$$
P_{x}=\{(u, y) \in X \times Y \mid u=x\}
$$

bestehende Partition von $W=X \times Y$ angewandt, und genauso folgt

(4) $\Sigma_{x \in X, y \in Y} a_{x y}=\Sigma_{x \in X} \Sigma_{y \in Y} a_{x y}$,

woraus sich umgekehrt auch (3) ergibt, wiederum unter Beachtung von (2).

8.5. Aus (1) resultiert der vertraute anschauliche Umgang mit endlichen Summen, Klammern dürfen beliebig gesetzt und weggelassen werden. ${ }^{7}$

Auch summierbare Familien, etwa in einem Banachraum, genügen dieser Grundregel, ${ }^{8}$ mit einer gewissen Einschränkung:

Die Existenz der großen Summe (links) muß vorausgesetzt werden, sie folgt nicht aus der Existenz der rechten Summen $\left(\sum_{X}\right.$ könnte immer $1-1$ sein).

Bei (2) und (3) denke man an eine Banachalgebra, etwa $\mathbb{R}$ oder $\mathbb{C}$.

Absolut konvergente Reihen sind (absolut) summierbare Familien, und sie sollten von vornherein als solche aufgefaßt werden.

\section{LITERATURHINWEISE}

Dedekinds Gesammelte mathematische Werke, herausgegeben von R. Fricke, E. Noether und Ö. Ore (Vieweg, Braunschweig 1932) werden von der Göttinger Bibliothek im Internet zur Verfügung gestellt [gdz.de].

Die Schriften von 1872 und 1888 finden sich in Band III, die Betrachtungen zum „Innerlichen und Äußerlichen“ in Band II (S. 54-55).

Zum Satz von Schröder-Bernstein siehe Band III (S. 447-448), auch Zermelos Anmerkung 2 (S. 451) in Cantors Ges. Abhandlungen (Springer, Berlin 1932). Der Brief an den Hamburger „Oberlehrer“ Dr. H. Keferstein wird auf Seite 490 kurz besprochen, erstaunlicherweise aber nicht wiedergegeben.

\footnotetext{
${ }^{7}$ Genau wie bei ganz allgemeinen direkten Summen und Produkten.

${ }^{8}$ K. Köngsberger: Analysis I, Springer, Berlin, ... 2001.

Die Regel (1) heißt dort "Großer Umordnungssatz“.
} 


\section{Dedekind und Die Grundlagen}

\section{Dedekinds Brief an Dr. Hans Keferstein vom 27. Februar 1890.}

Wiedergegeben mit freundlicher Erlaubnis der Niedersächsischen Staats- und Universitätsbibliothek Göttingen. Signatur: Cod. Ms. Dedekind XIII:19.

Näheres über die Vorgeschichte findet sich in dem Buch From Frege to Gödel von Jean van Heijenoort (Harvard University Press, 1967).

\section{Hochgeehrter Herr Doktor!}

Für Ihren freundlichen Brief vom 14. d. M. und die darin ausgesprochene Bereitwilligkeit, meiner Entgegnung Gehör zu verschaffen, sage ich Ihnen meinen besten Dank. Doch möchte ich Sie bitten, in dieser Sache Nichts zu übereilen und erst dann einen Entschluß zu fassen, nachdem Sie, wenn Sie Zeit dazu haben, die wichtigsten Erklärungen und Beweise in meiner Zahlenschrift noch einmal genau gelesen und durchdacht haben. Ich halte es nämlich für höchst wahrscheinlich, daß Sie sich dann in allen Punkten zu meiner Auffassung und Behandlung des Gegenstandes bekehren werden, und gerade hierauf würde ich bei weitem den größten Wert legen, weil ich überzeugt bin, daß Sie wirklich ein tiefes Interesse für die Sache hegen.

Um diese Annäherung wo möglich zu befördern, möchte ich Sie bitten, dem folgenden Gedankengange, der die Genesis meiner Schrift darstellt, Ihre Aufmerksamkeit zu schenken. Wie ist meine Schrift entstanden? Gewiß nicht in einem Tage, sondern sie ist eine nach langer Arbeit aufgebaute Synthese, die sich auf eine vorausgehende Analyse der Reihe der natürlichen Zahlen stützt, so wie diese sich, gewissermaßen erfahrungsmäßig, unserer Betrachtung darbietet. Welches sind die von einander unabhängigen Grundeigenschaften dieser Reihe $N$, d. h. diejenigen Eigenschaften, welche sich nicht aus einander ableiten lassen, aus denen aber alle anderen folgen? Und wie muß man diese Eigenschaften ihres spezifisch arithmetischen Charakters entkleiden, der Art, daß sie sich allgemeinen Begriffen und solchen Tätigkeiten des Verstandes unterordnen, ohne welche überhaupt kein Denken möglich ist, mit welchen aber auch die Grundlage gegeben ist für die Richtigkeit und Vollständigkeit der Beweise, wie für die Bildung widerspruchsfreier Begriffs-Erklärungen.

Stellt man die Frage in dieser Weise, so wird man, wie ich glaube, mit Gewalt auf folgende Thatsachen gedrängt:

1) Die Zahlenreihe $N$ ist ein System von Individuen oder Elementen, die Zahlen heißen. Dies führt zur allgemeinen Betrachtung von Systemen ( $\$ 1$ meiner Schrift).

2) Die Elemente des Systems $N$ stehen in gewisser Beziehung zu einander, es herrscht eine gewisse Ordnung, die zunächst darin besteht, daß zu jeder bestimmten Zahl $n$ eine bestimmte Zahl $n^{\prime}$, die folgende oder nächst größere Zahl 
$\underline{\text { gehört. Dies führt zur Betrachtung des allgemeinen Begriffes einer Abbildung }}$

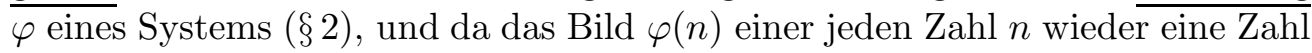
$n^{\prime}$, also $\varphi(N)$ Teil von $N$ ist, so handelt es sich hier um eine Abbildung $\varphi$ eines Systems $N$ in sich selbst, welche also allgemein zu untersuchen ist (§4).

3) Auf verschiedene Zahlen $a, b$ folgen auch verschiedene Zahlen $a^{\prime}, b^{\prime}$. Die Abbildung $\varphi$ hat also den Charakter der Deutlichkeit oder $\underline{\text { Ahhnlichkeit }}(\S 3)$.

4) Nicht jede Zahl ist eine folgende Zahl $n^{\prime}$, d. h. $\varphi(N)$ ist echter Teil von $N$, und hierin besteht in Verbindung mit dem Vorhergehenden die Unendlichkeit der Zahlenreihe $(\S 5)$.

5) Und zwar ist 1 die einzige Zahl, welche sich nicht in $\varphi(N)$ findet. Hiermit sind diejenigen Tatsachen aufgezählt, in welchen Sie (S.124. Z.8-14) den vollständigen Charakter eines geordneten einfach unendlichen Systems erblicken.

6) Aber ich habe in meiner Entgegnung (III) gezeigt, daß diese Thatsachen noch lange nicht ausreichen, um das Wesen der Zahlenreihe $N$ vollständig zu erfassen. Alle diese Tatsachen würden auch noch für jedes System $S$ gelten, welches außer der Zahlenreihe $N$ noch ein System $T$ von beliebigen anderen Elementen enthält, auf welches die Abbildung $\varphi$ sich stets so ausdehnen läßt, daß sie den Charakter der Ähnlichkeit behält, und daß $\varphi(T)=T$ wird. Aber ein solches System ist offenbar etwas ganz Anderes als unsere Zahlenreihe $N$, und ich könnte es so wählen, daß in ihm kaum ein einziger der arithmetischen Sätze bestehen bliebe. Was muß also zu den bisherigen Thatsachen noch hinzu kommen, um unser System von solchen fremden, alle Ordnung störenden Eindringlingen wieder zu reinigen? Dies war einer der schwierigsten Punkte in meiner Analyse, und seine Überwindung hat ein langes Nachdenken erfordert. Setzt man die Kenntnis der natürlichen Zahlenreihe $N$ schon voraus und erlaubt sich demgemäß eine arithmetische Ausdrucksweise, so hat man ja leichtes Spiel; man braucht nur zu sagen: ein Element gehört dann und nur dann der Reihe $N$ an, wenn ich, von dem Element 1 ausgehend, durch beständig wiederholtes Weiterzählen, d. h. durch eine endliche Anzahl von Wiederholungen der Abbildung $\varphi$ (vergl. den Schluß von 131 meiner Schrift) wirklich einmal zu dem Element $n$ gelange, während ich auf diese Weise niemals zu einem der Reihe $N$ fremden Element $t$ gelange. Aber dieser Weg, den Unterschied zwischen den aus $S$ wieder auszutreibenden Elementen $t$ und den allein beizubehaltenden Elementen $n$ zu charakterisieren, ist doch für unsere Zwecke gänzlich unbrauchbar, es entsteht ja ein circulus vitiosus der schlimmsten und auffälligsten Art. Die bloßen Worte „endlich einmal hinkommen" thun es natürlich auch nicht, sie würden nicht mehr helfen als etwa die Worte „karam sipo tatura“, die ich augenblicklich erfinde, ohne ihnen einen deutlich erklärten Sinn zu geben. Also: wie kann ich, ohne irgend welche arithmetische Kenntnis voraus zu setzen, den Unterschied zwischen den Elementen $n$ und $t$ unfehlbar begrifflich bestimmen? Ganz 
allein durch die Betrachtung der Ketten (37, 44 meiner Schrift), durch diese aber auch vollständig! Will ich meinen Kunstausdruck „Kette“ vermeiden, so werde ich sagen: ein Element $n$ von $S$ gehört dann und nur dann der Reihe $N$ an, wenn $n$ Element jedes solchen Theils $K$ von $S$ ist, welcher die doppelte Eigenschaft besitzt, daß das Element 1 in $K$ enthalten ist, und daß das Bild $\varphi(K)$ Teil von $K$ ist. In meiner Kunstsprache: $N$ ist die Gemeinheit $1_{0}$ oder $\varphi_{0}(1)$ aller derjenigen Ketten $K$ (in $S$ ), in denen das Element 1 enthalten ist. Erst hierdurch ist der vollständige Charakter der Reihe festgestellt. - Hierzu bemerke ich beiläufig Folgendes: Die „Begriffsschrift“ und die „Grundlagen der Arithmetik" von Frege sind zum ersten Male im letzten Sommer (1889) auf kurze Zeit in meine Hände gelangt, und ich habe mit Vergnügen gesehen, daß seine Art, das mittelbare Folgen eines Elementes auf ein anderes in einer Reihe zu erklären, im Wesentlichen mit meinen Ketten-Begriffen $(37,44)$ übereinstimmt, man muß sich nur durch seine etwas unbequeme Ausdrucksweise nicht zurückschrecken lassen.

7) Nachdem in meiner Analyse der wesentliche Charakter des einfach zusammenhängenden Systems, dessen abstrakter Typus die Zahlenreihe $N$ ist, erkannt war $(71,73)$, fragte es sich: existiert überhaupt ein solches System in unserer Gedankenwelt? Ohne den logischen Existenz-Beweis würde es immer zweifelhaft bleiben, ob nicht der Begriff eines solchen Systems vielleicht innere Widersprüche enthält. Daher die Notwendigkeit solcher Beweise (66, 72 meiner Schrift).

8) Nachdem auch dies festgestellt war, fragte es sich: liegt in dem Bisherigen auch eine ausreichende Beweismethode, um Sätze, die für alle Zahlen gelten sollen, allgemein zu beweisen? Ja! Der berühmte Induktions-Beweis ruht auf der sicheren Grundlage des Ketten-Begriffs (59, 60, 80 meiner Schrift).

9) Endlich: Ist es auch möglich, die Definitionen für Zahlen-Operationen widerspruchsfrei für alle Zahlen aufzustellen? Ja! Dies wird durch den Satz 126 in meiner Schrift in der That geleistet.

Damit war die Analyse beendigt, und der synthetische Aufbau konnte beginnen; es hat mir doch noch Mühe genug gemacht! Auch der Leser meiner Schrift hat es wahrlich nicht leicht; außer dem gesunden Menschenverstande gehört auch noch ein sehr starker Wille dazu, um Alles vollständig durchzuarbeiten.

Ich wende mich nun noch zu einigen Stellen Ihrer Abhandlung, die ich in meiner neulichen Entgegnung nicht erwähnt habe, weil sie weniger wichtig sind; vielleicht werden aber meine darauf bezüglichen Bemerkungen noch einiges zur Klärung der Sache beitragen.

a) S.121. Z.19. Weshalb wird hier von einem Theile gesprochen? Eine Anzahl schreibe ich später (161 meiner Schrift) jedem endlichen Systeme und nur einem solchen zu. 
b) S.122. Z.8. Hier findet sich eine Verwechselung zwischen Abbildung und Bild; statt „Abbildung $\bar{\varphi}\left(S^{\prime}\right)$ " müßte es heißen „Abbildung $\bar{\varphi}$ des Systems

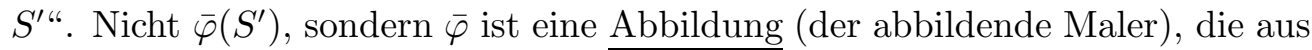

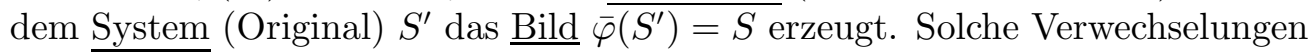
können aber bei unserer Untersuchung recht gefährlich werden.

c) S.123. Z.29-31. Diese Worte mögen vielleicht auf Frege passen, auf mich gewiß nicht. Die Zahl 1 als Grundelement der Zahlenreihe wird von mir mit vollkommener Bestimmtheit erklärt in 71, 73, und die Anzahl 1 ergiebt sich ebenso im Satze 164 als Folge der allgemeinen Erklärung 161. Hierzu darf gar Nichts weiter hinzugefügt werden, wenn nicht eine Trübung eintreten soll.

d) S.123. Z.29-31. Dies ist schon durch die vorhergehende Bemerkung c) erledigt. Und wie würde wohl die größere Sicherheit und die geringere Weitläufigkeit sich thatsächlich gestalten?

e) S.124. Z.21-24. Der Sinn dieser Zeilen (sowie der vorhergehenden und nachfolgenden) ist mir nicht ganz deutlich. Soll hier etwa der Wunsch ausgesprochen sein, meine Definition der Zahlenreihe und der Aufeinanderfolge des Elementes $n^{\prime}$ auf das Element $n$ wo möglich anzulehnen an eine anschauliche Reihe? Dem würde ich mich mit größter Bestimmtheit widersetzen, weil sofort die Gefahr entsteht, aus einer solchen Anschauung vielleicht unbewußt auch Sätze als selbstverständlich zu entnehmen, die vielmehr ganz abstrakt aus der logischen Definition von $N$ abgeleitet werden müssen. Wenn ich $n^{\prime}$ das auf $n$ folgende Element nenne (73), so soll das lediglich ein neuer Kunstausdruck sein, durch dessen Benutzung ich nur einige Abwechslung in meine Sprache bringe; diese Sprache würde noch einförmiger und abschreckender klingen, wenn ich auf diese Abwechslung verzichten und $n^{\prime}$ immer nur das Bild $\varphi(n)$ von $n$ nennen müßte. Aber der eine Ausdruck soll genau dasselbe bedeuten wie der andere.

f) S.124. Z.33 - S.125. Z.7. Das in der dritten Zeile meiner Erklärung 73 gewählte Wort „lediglich“ soll doch offenbar die einzige Einschränkung bezeichnen, welcher das unmittelbar vorhergehende Wort „gänzlich“ zu unterwerfen ist; ließe man diese Einschränkung fallen, nähme also das Wort „gänzlich“ in seinem vollen Sinne, so würde auch die Unterscheidbarkeit der Elemente wegfallen, welche doch für den Begriff des einfach unendlichen Systems unentbehrlich ist. Mir scheint daher dieses „lediglich“ durchaus nicht überflüssig, sondern notwendig zu sein. Ich verstehe nicht, wie dies einen Anstoß erregen kann.

Indem ich meinen zu Anfang geäußerten Wunsch wiederhole und Sie bitte, die Ausführlichkeit meiner Erörterungen entschuldigen zu wollen, verbleibe ich mit größter Hochachtung

Braunschweig, Ihr ergebenster

27. Februar 1890. R. Dedekind.

Petrithorpromenade 24 . 


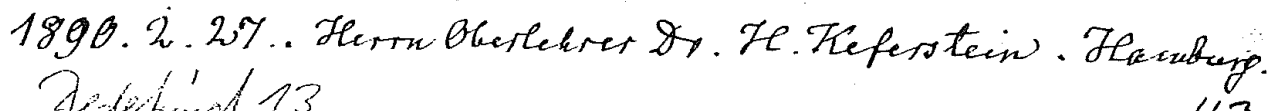
Delow 13

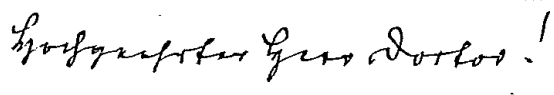

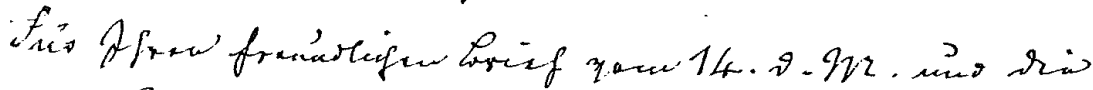

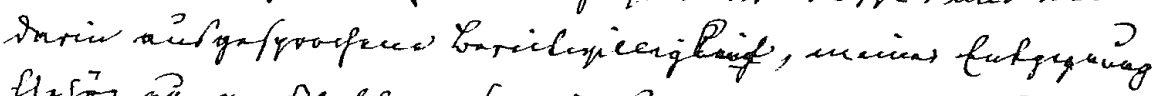

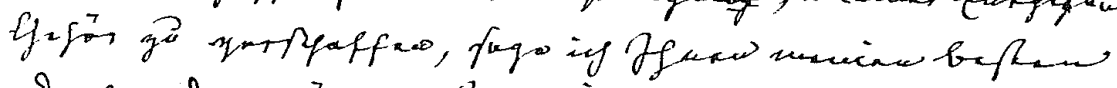

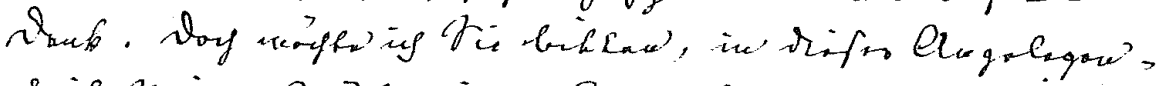

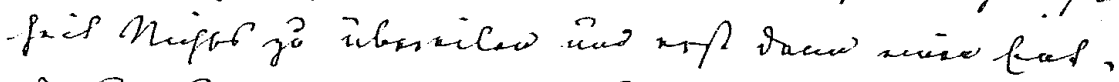

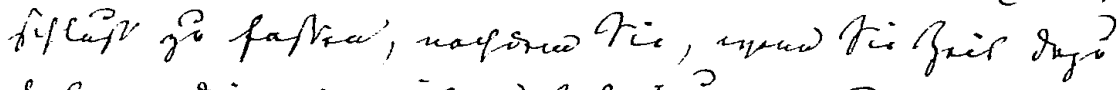

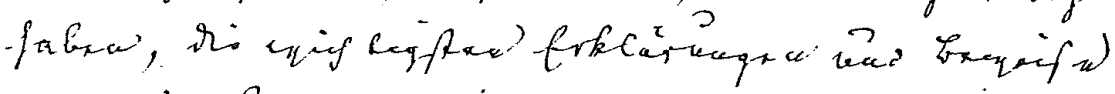

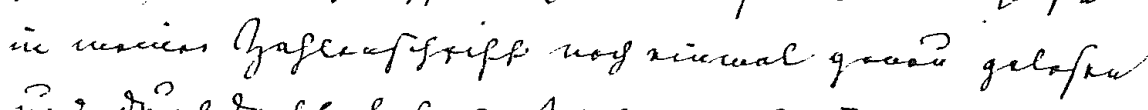

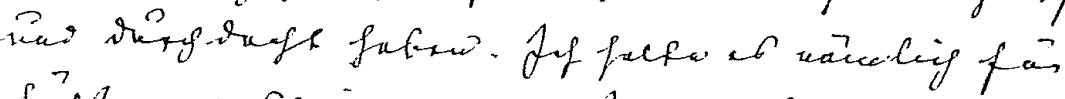

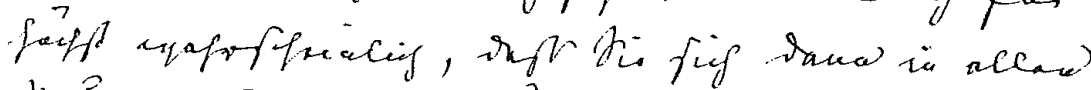

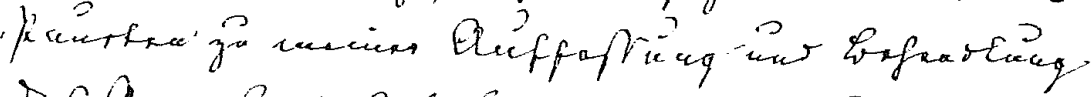

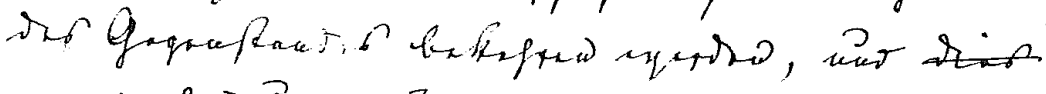

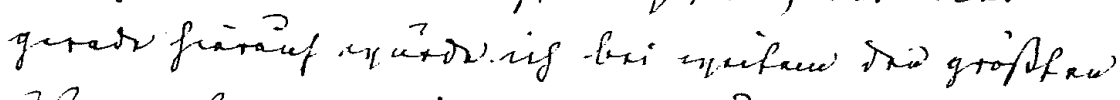

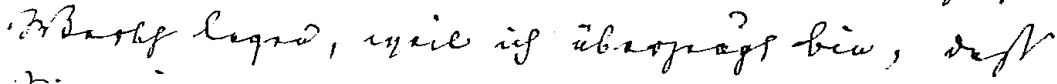

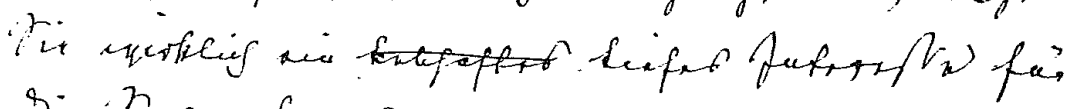
Ri itago frgron.

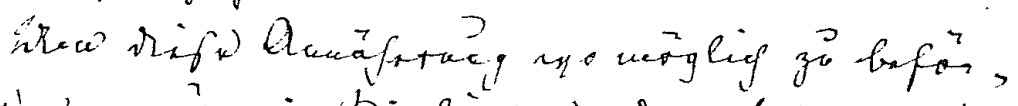

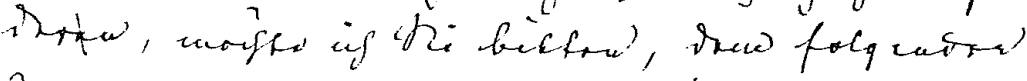

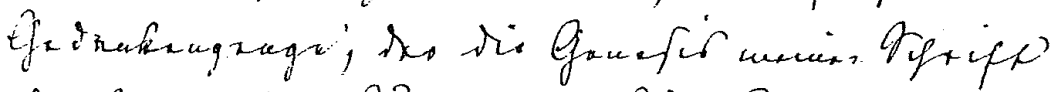

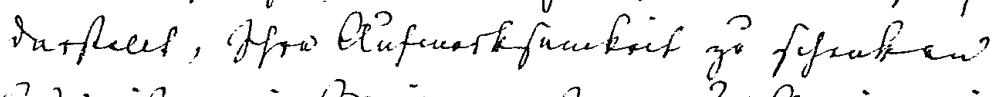

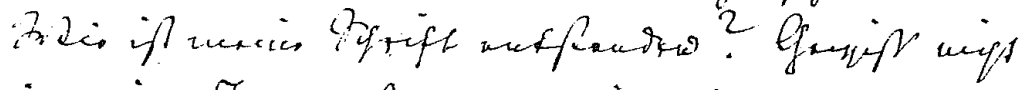

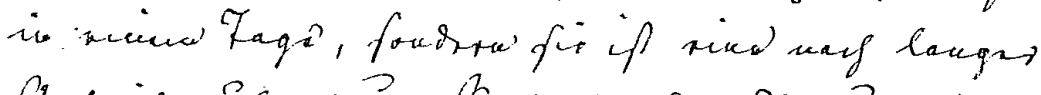

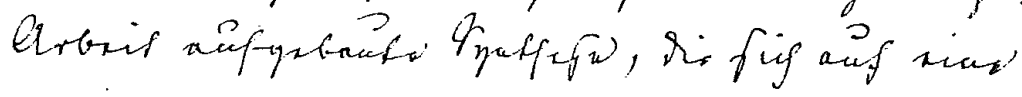


2.

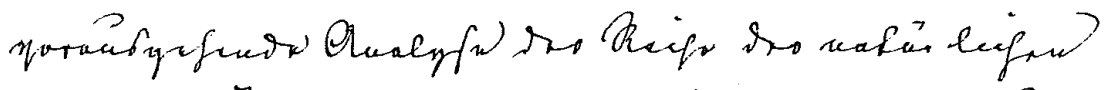

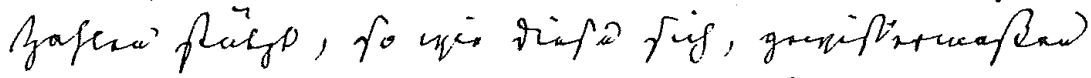

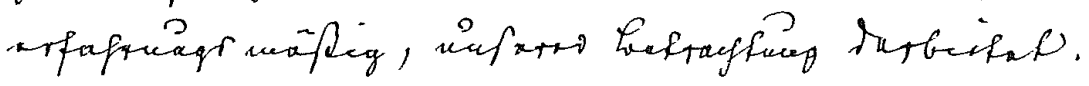

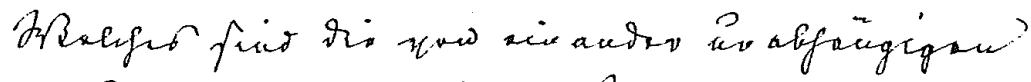

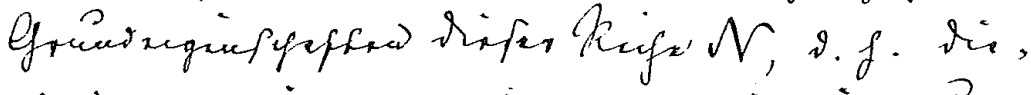

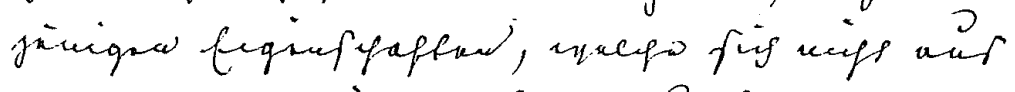

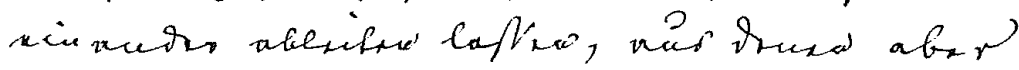

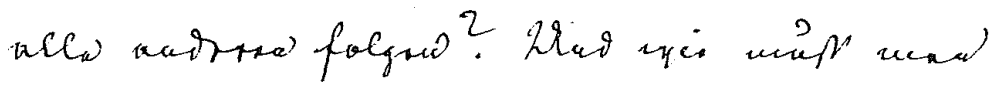

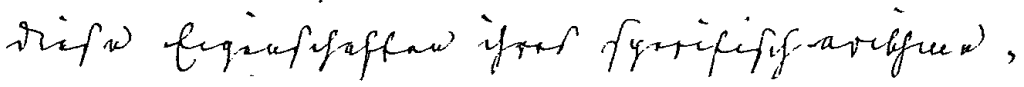

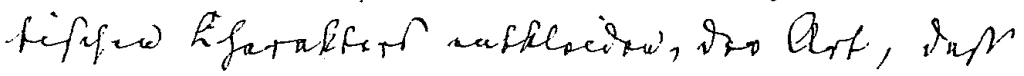

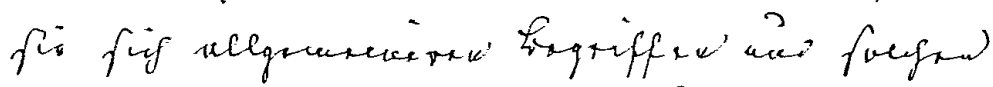

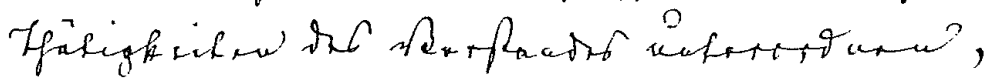

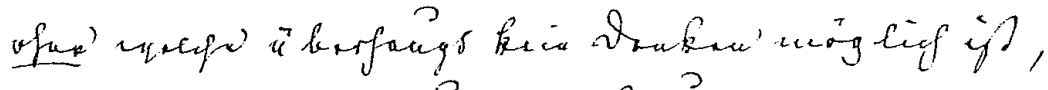

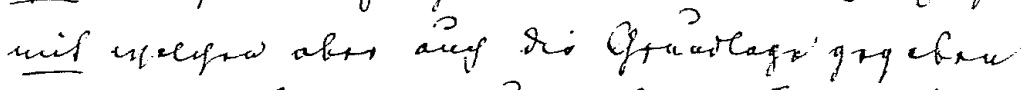

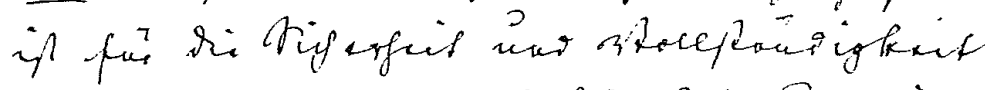

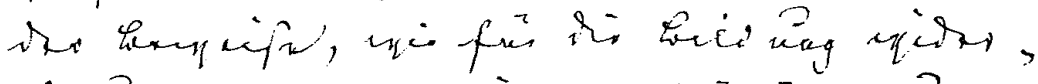

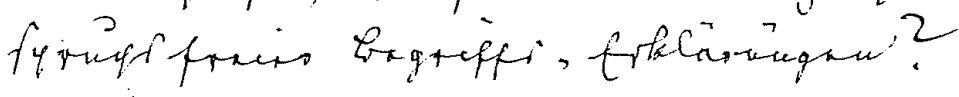

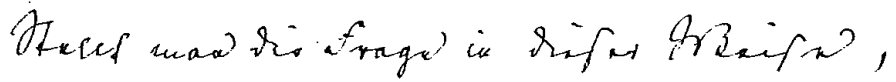

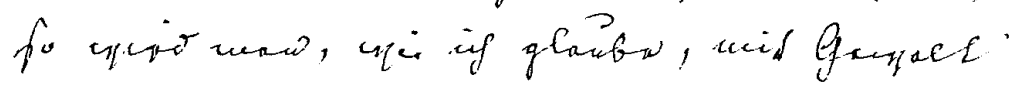

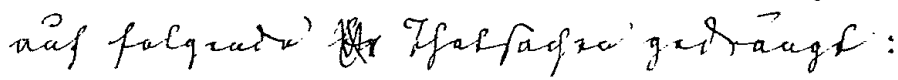

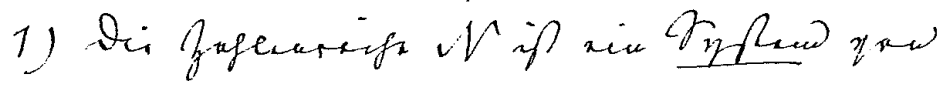

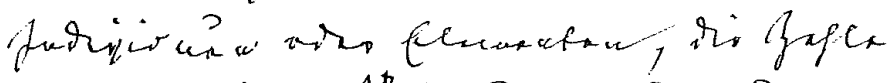

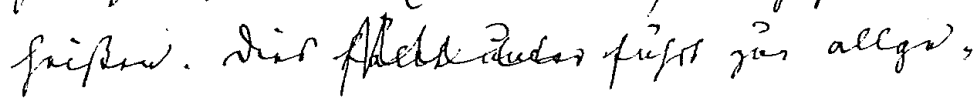


3.

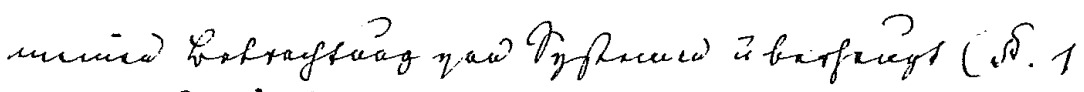
mevien Dytiff?

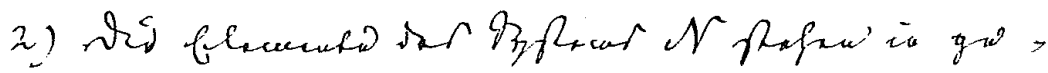

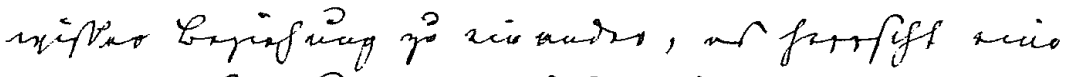

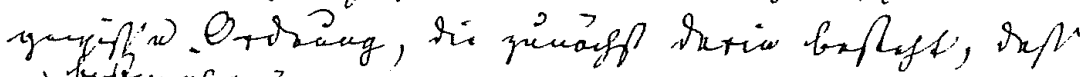

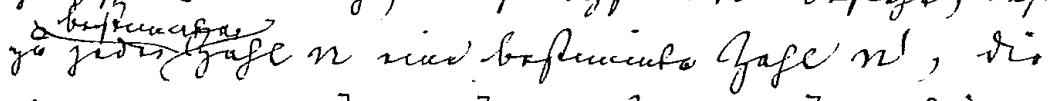

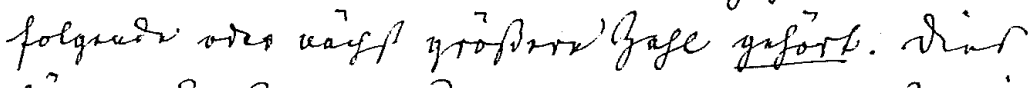

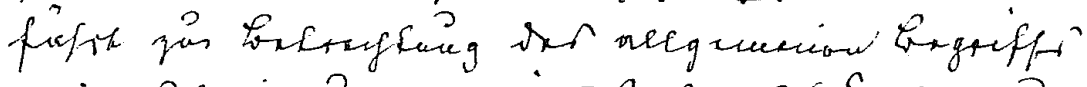

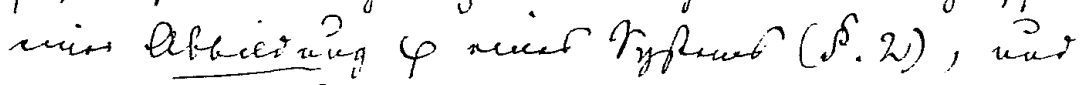

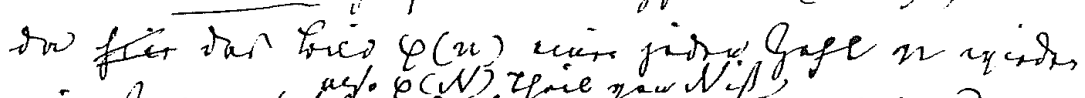

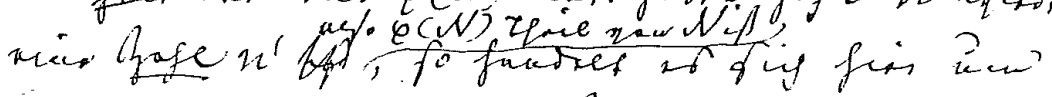

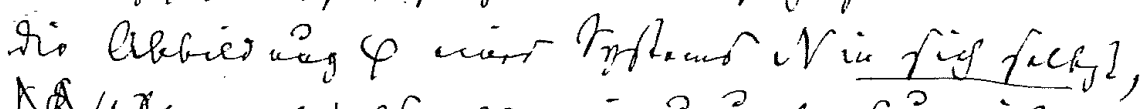

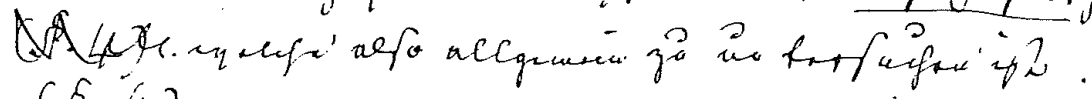
(6.4)

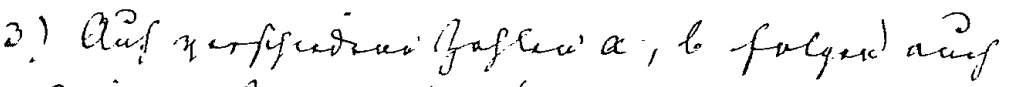

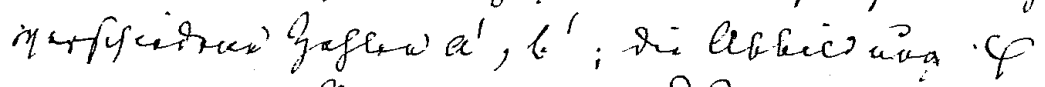

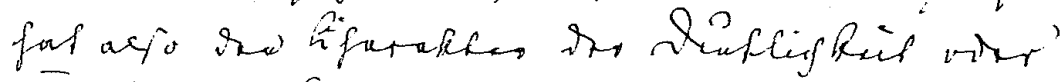

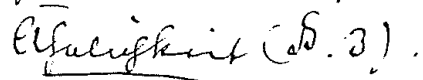

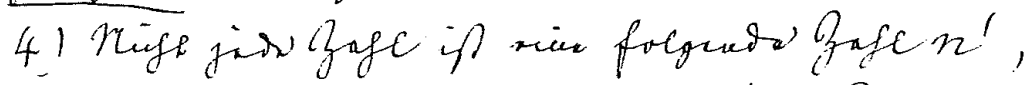

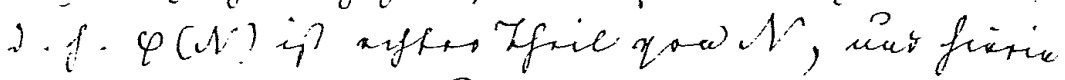

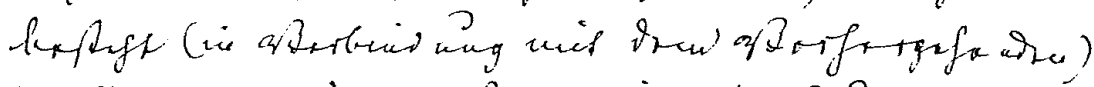

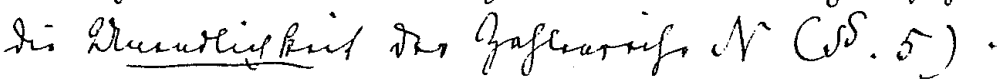

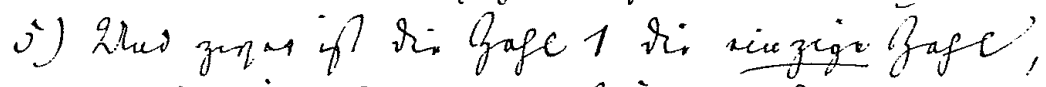

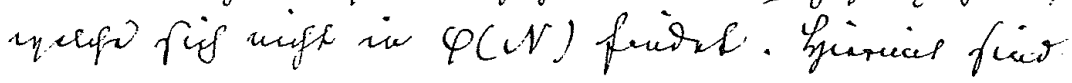




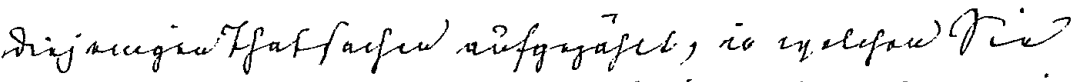

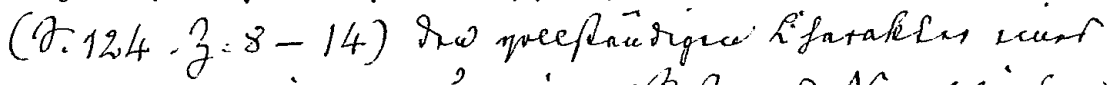

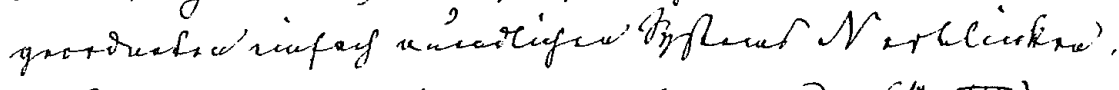

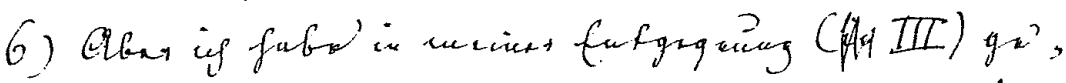

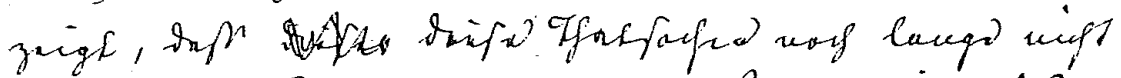

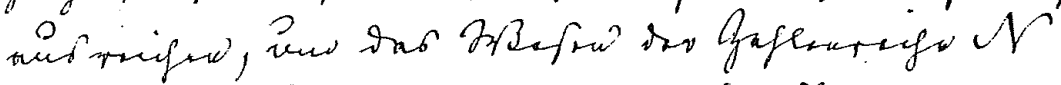

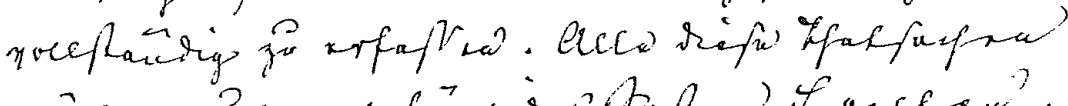

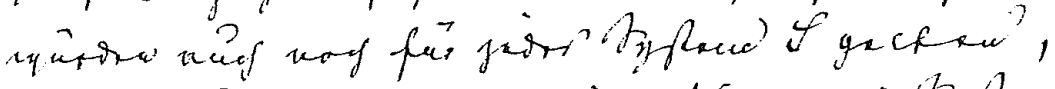

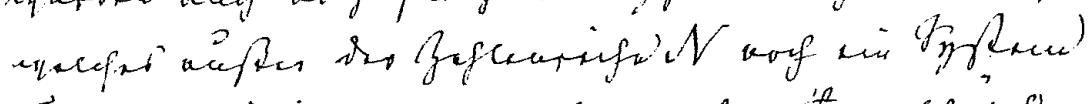

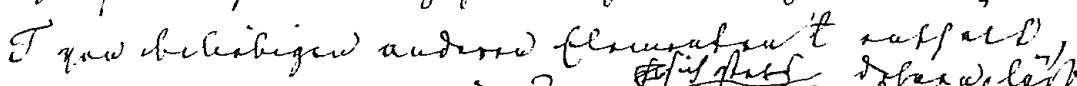

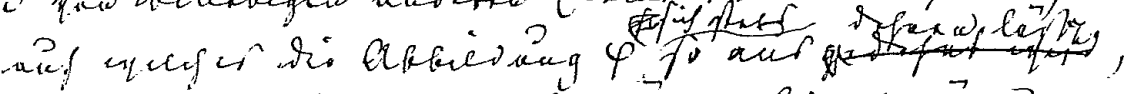

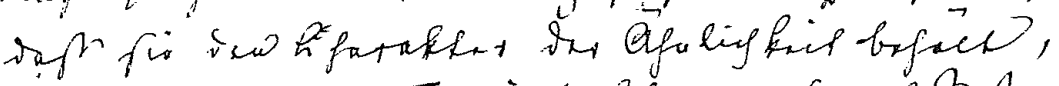

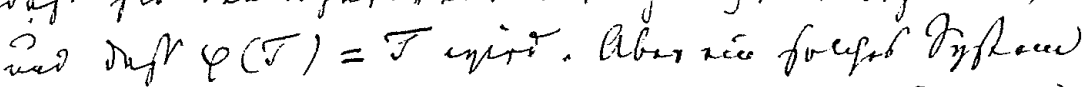

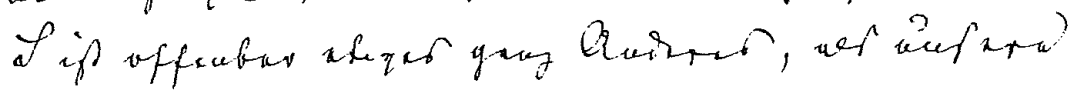

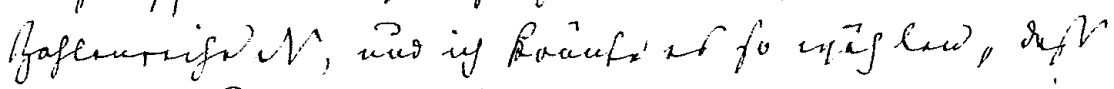

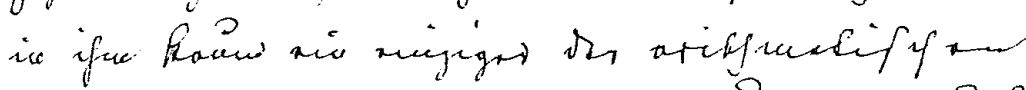

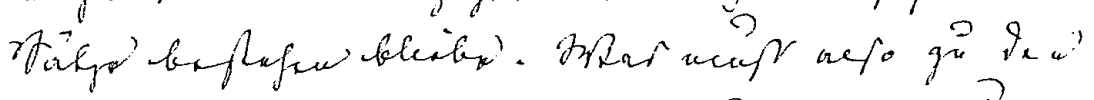

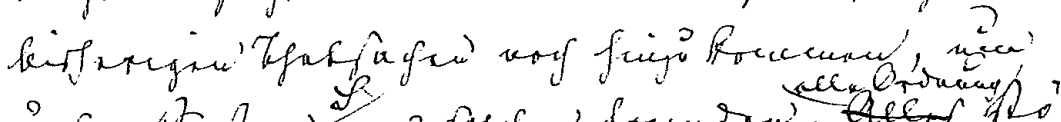

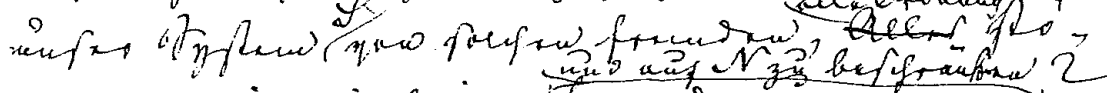

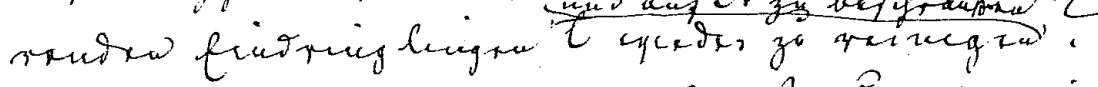

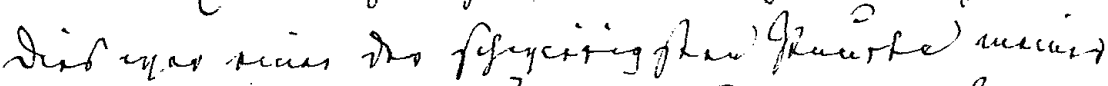

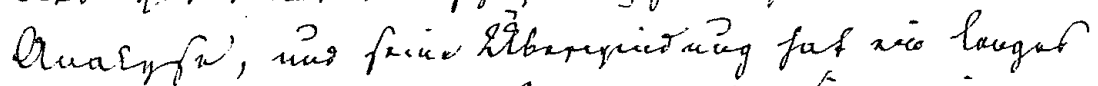

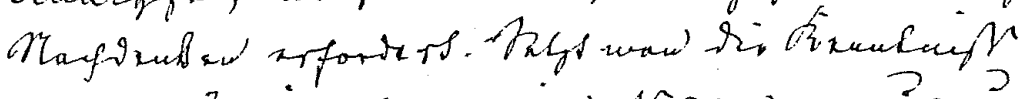

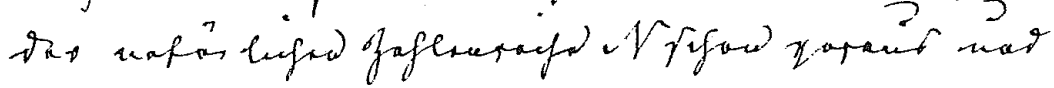


Dedekind 13

$1 / 3=5$

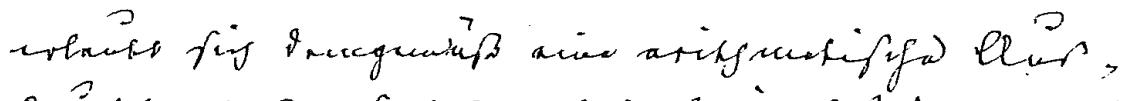

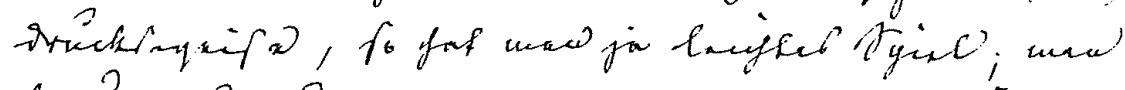

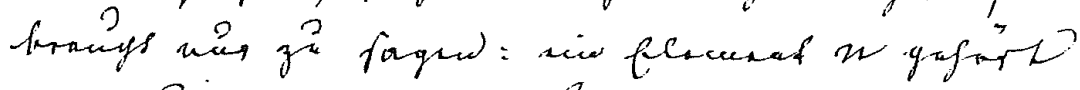

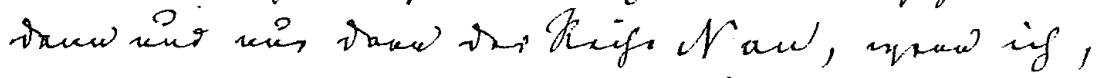

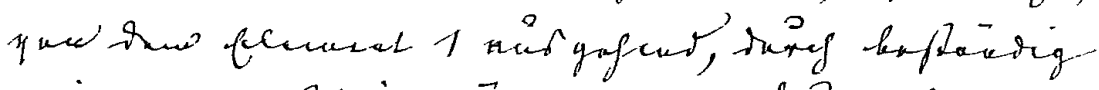

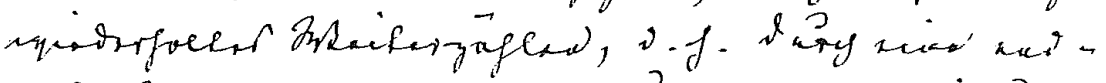

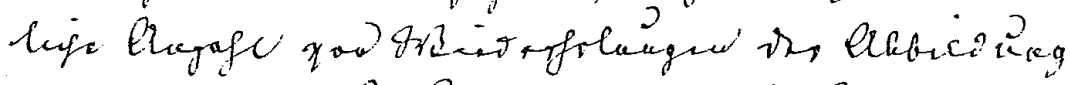

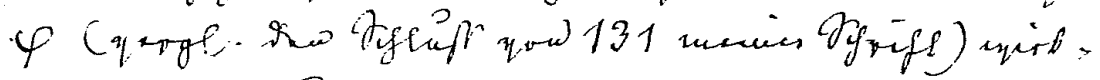

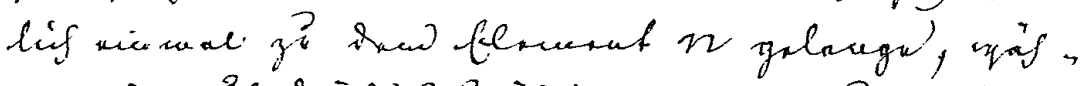

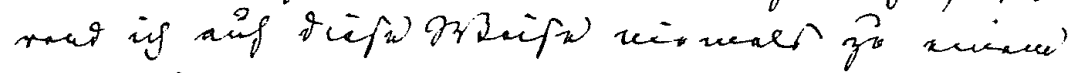

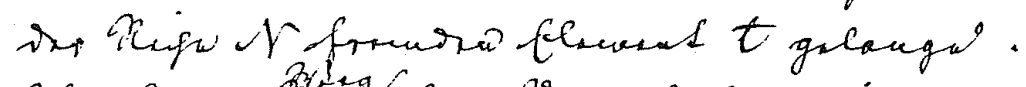

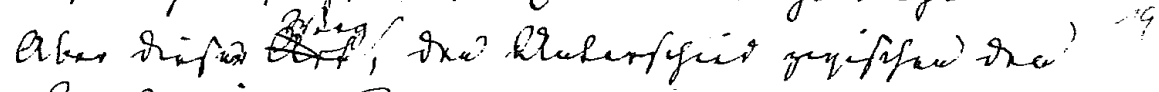

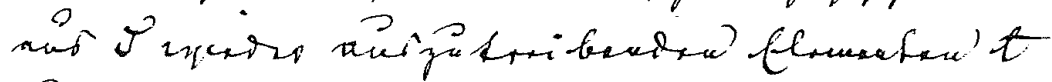

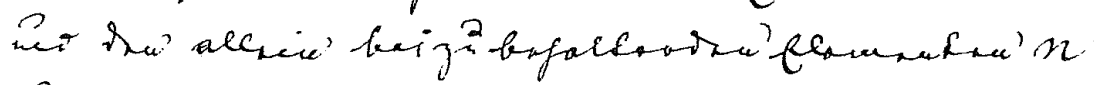

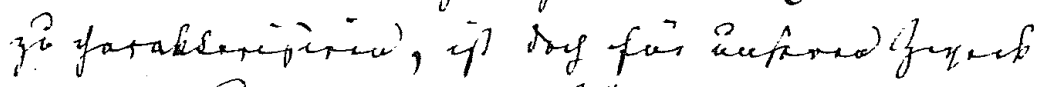

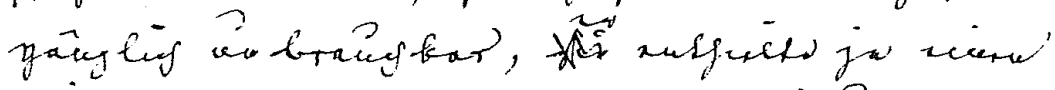

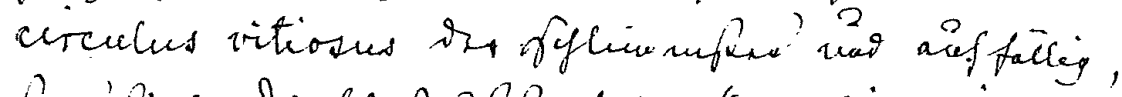

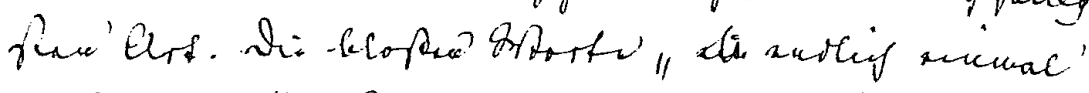

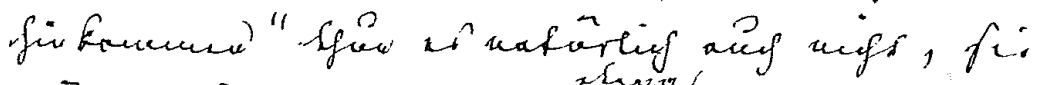

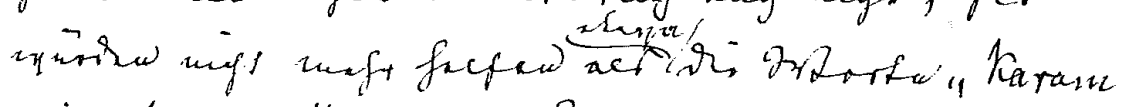

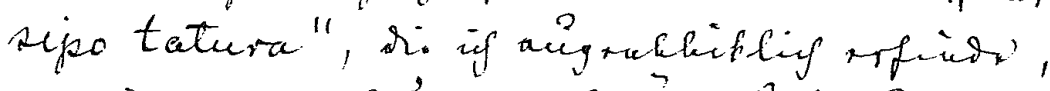

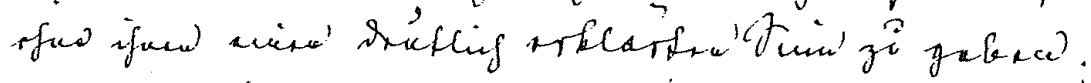

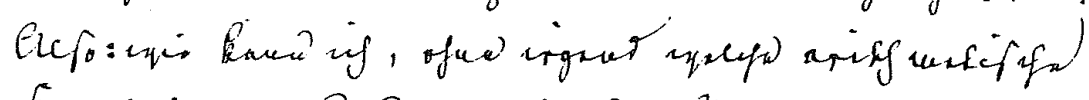

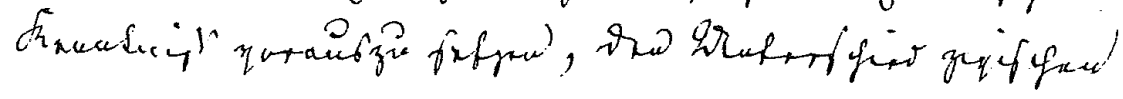




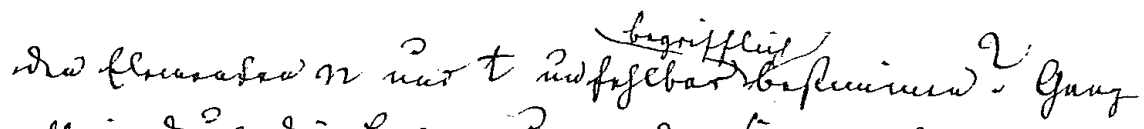

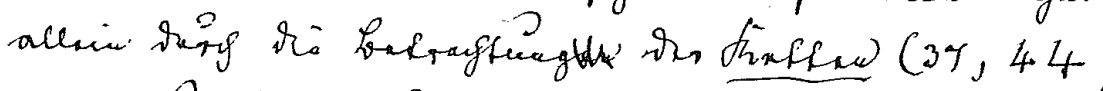

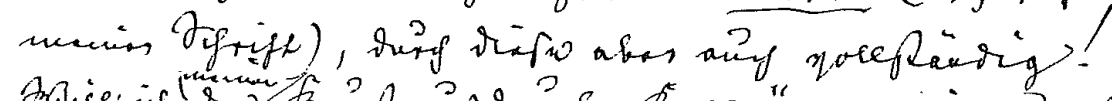

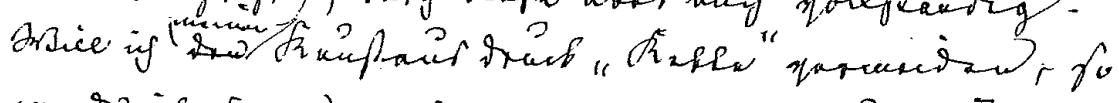

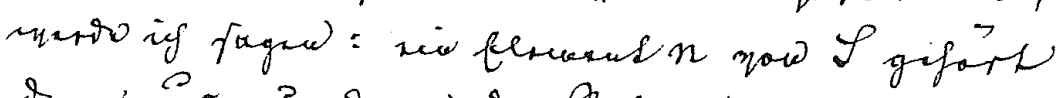

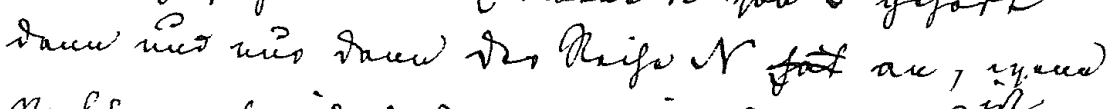

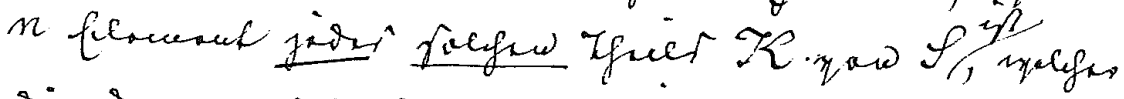

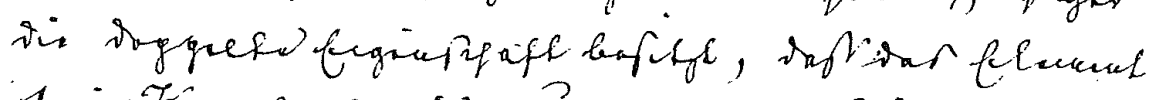

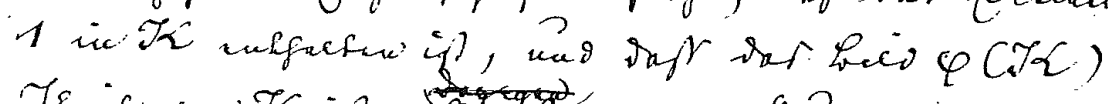

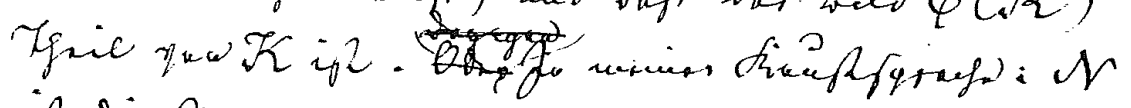

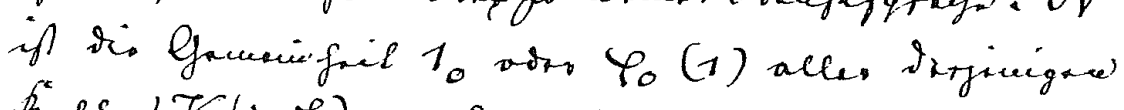

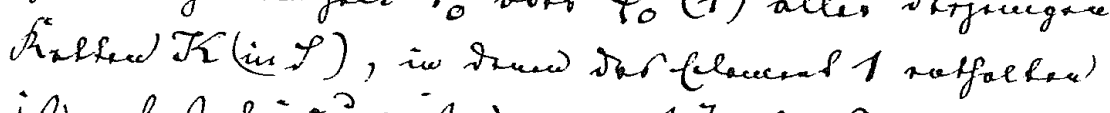

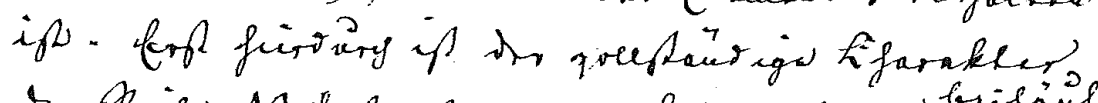

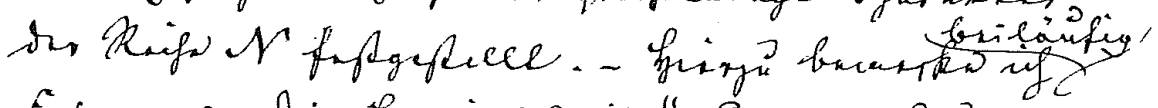

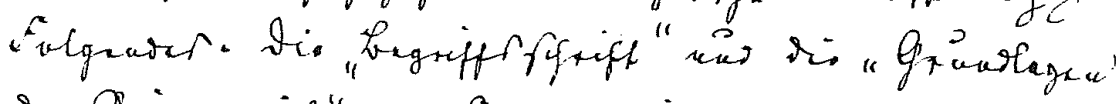

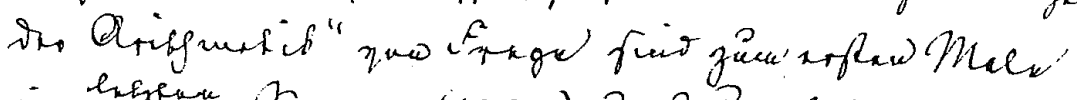

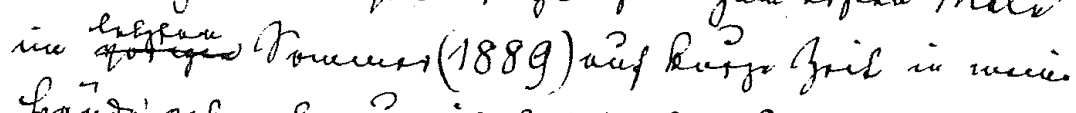

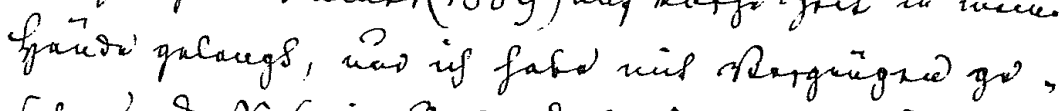

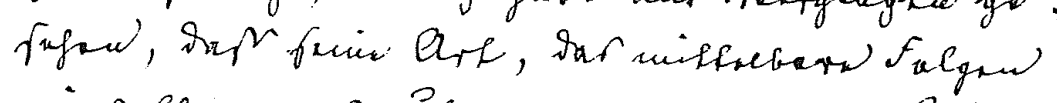

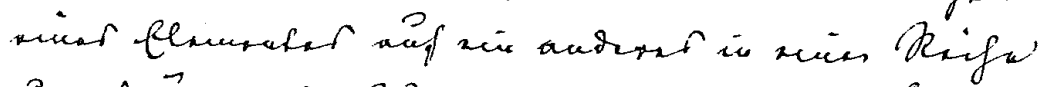

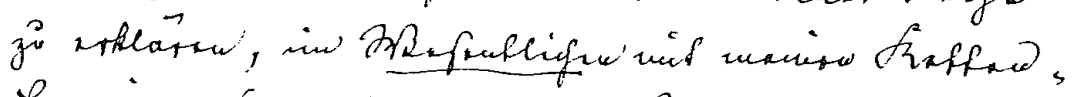

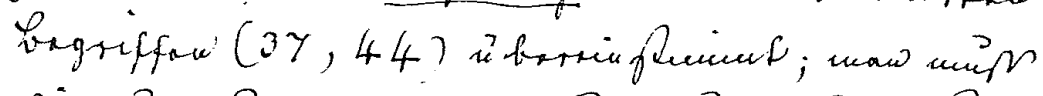

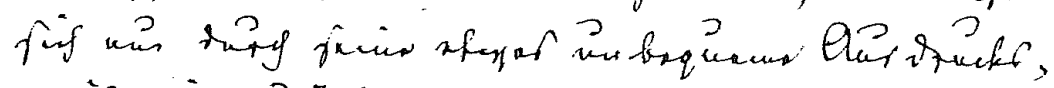

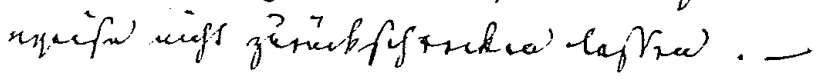


7

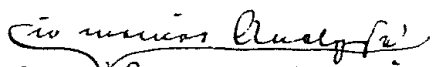

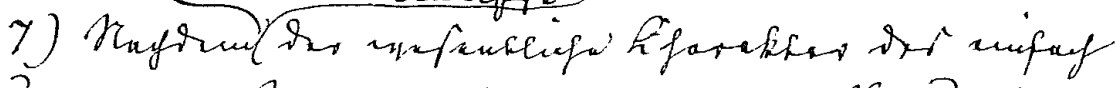

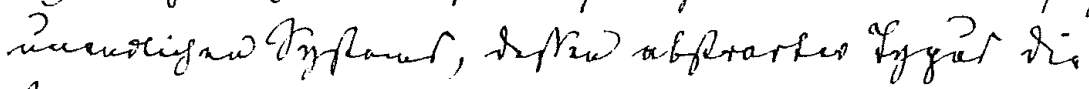

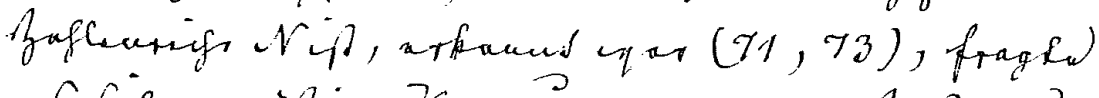

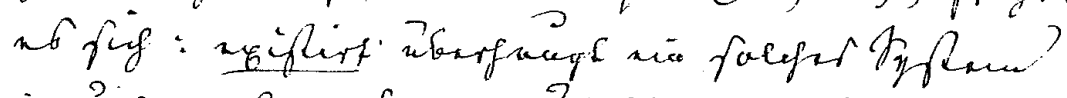

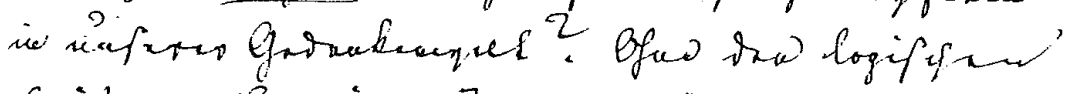

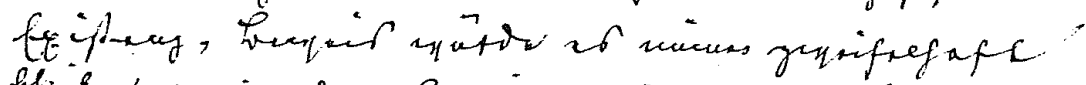

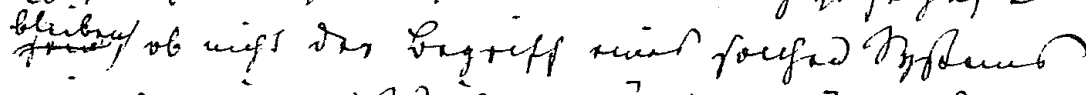

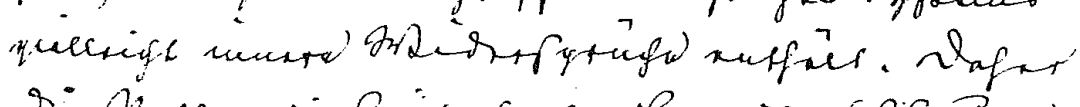

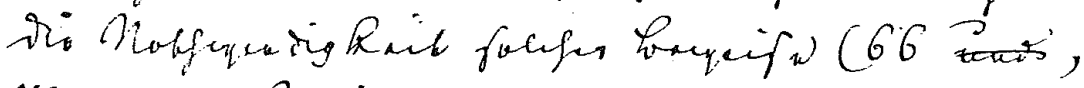
72. ansie, (iffift).

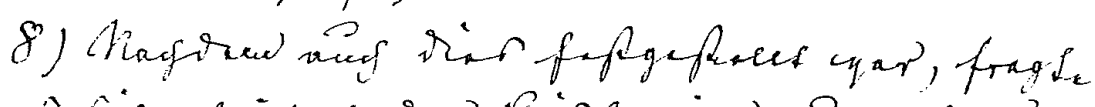

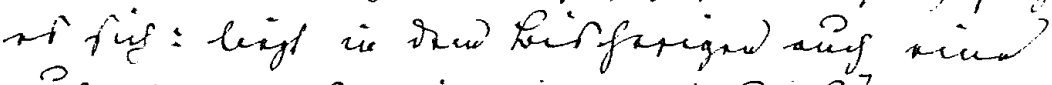

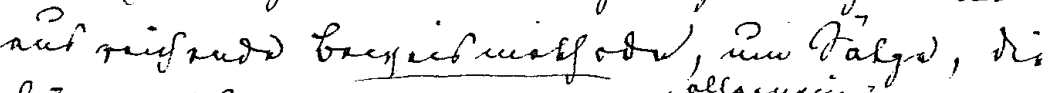

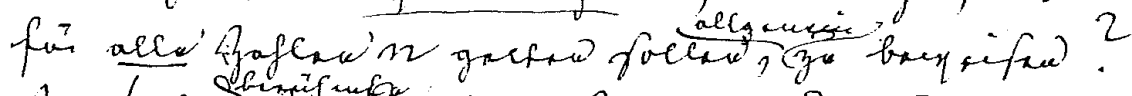

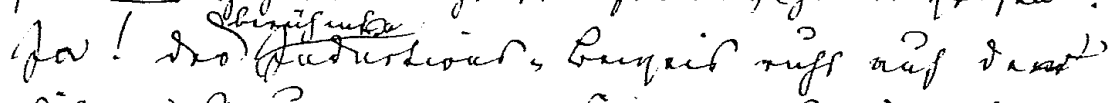

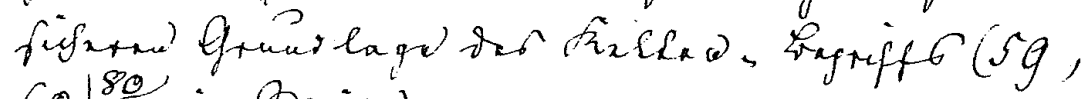
Go, (E⿱

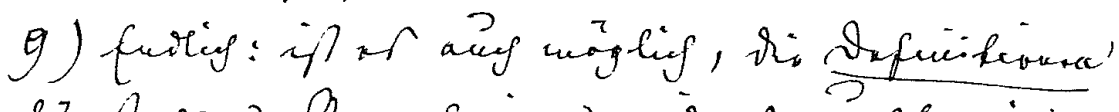

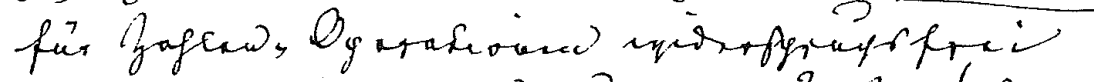

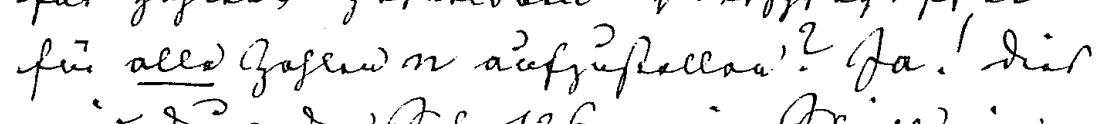

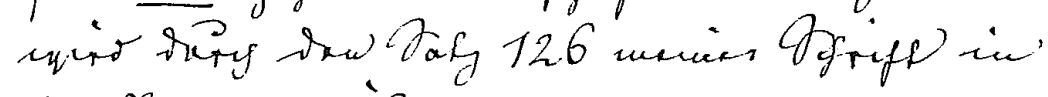
ixo 'Igut quarject. -

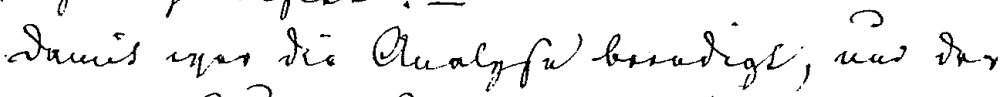

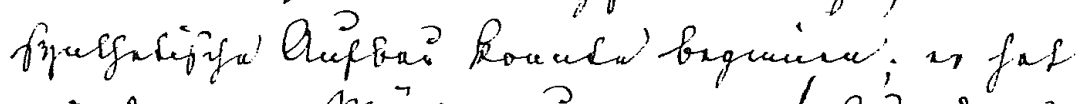

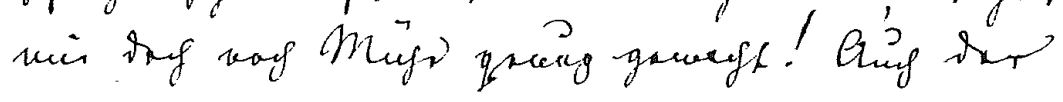


8.

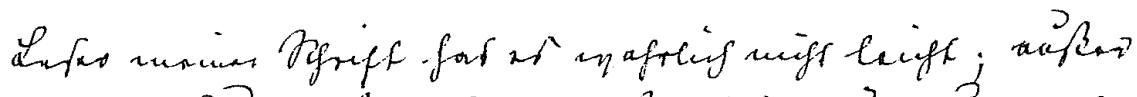

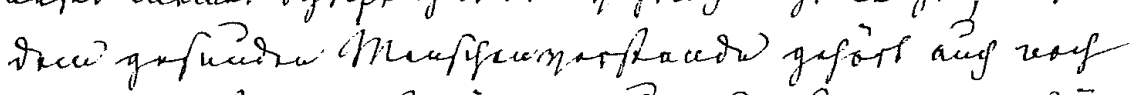

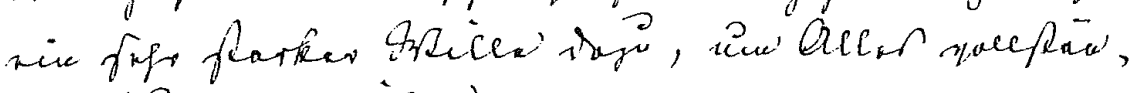
rig Vintigio arkitaci. -

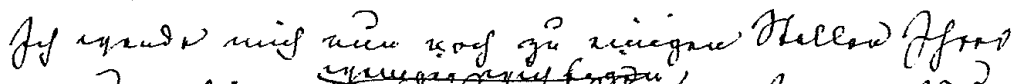

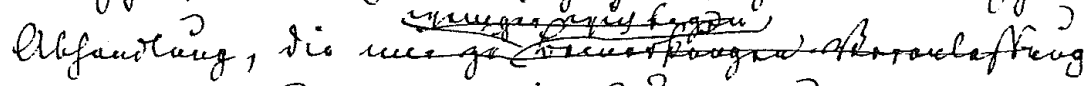

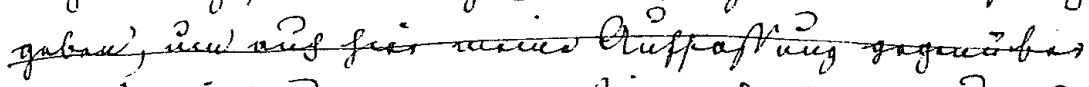

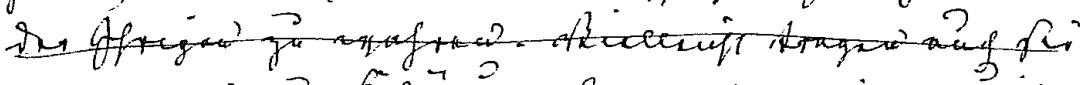

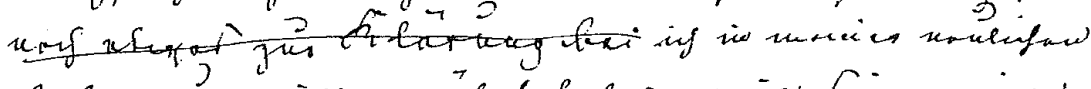

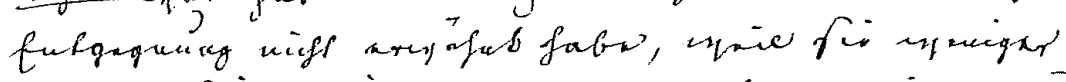

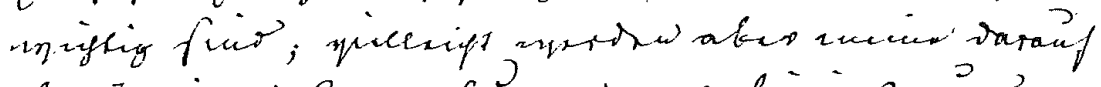

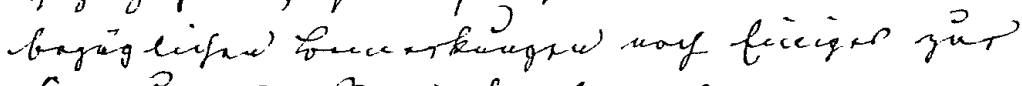

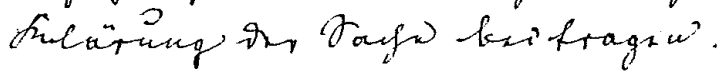

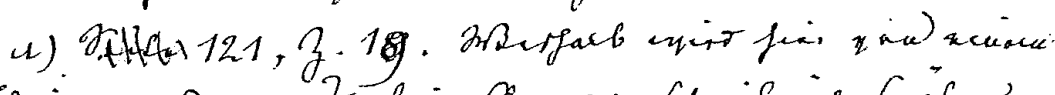

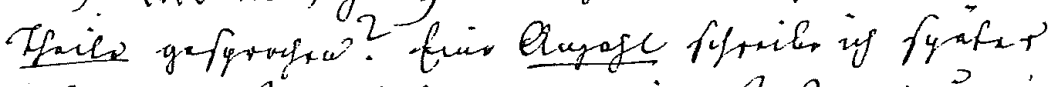

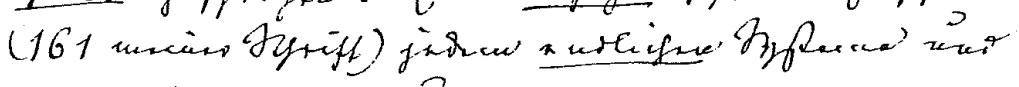

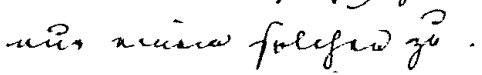

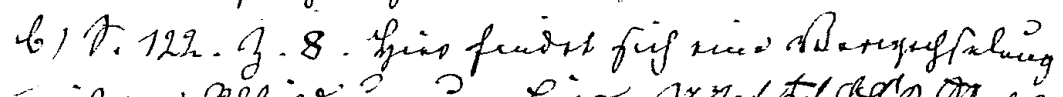

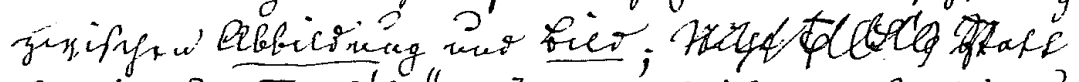

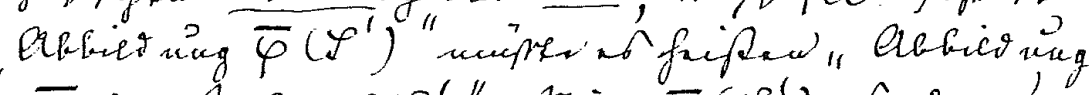

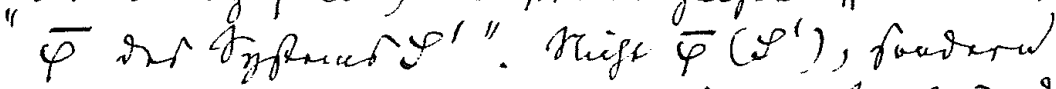

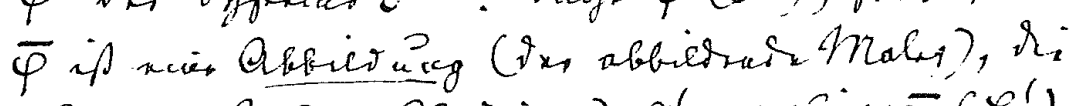

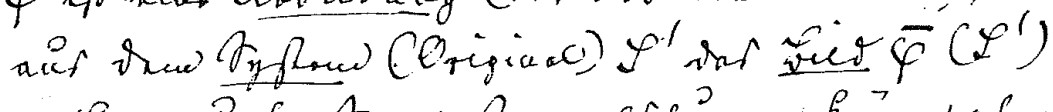

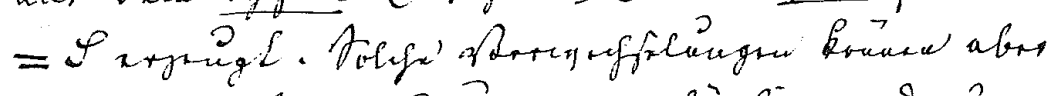

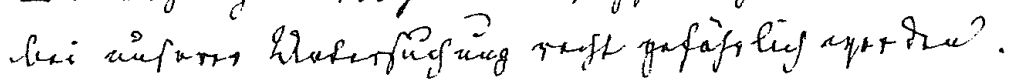


Deatebinat 18

2) $9=43$

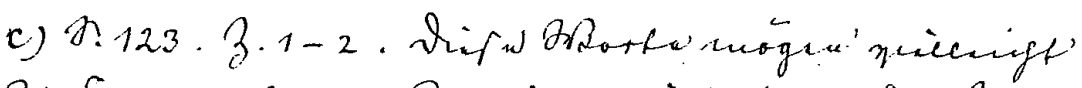

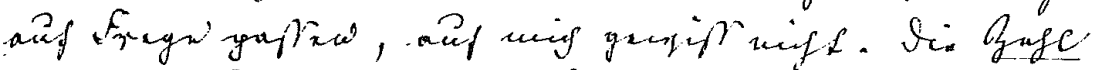

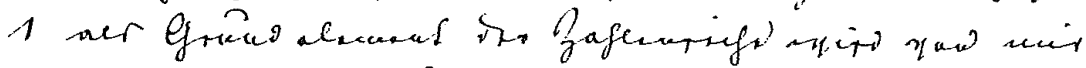

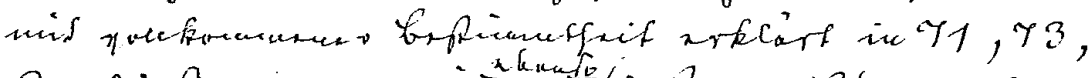

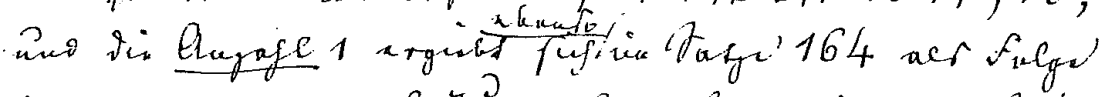

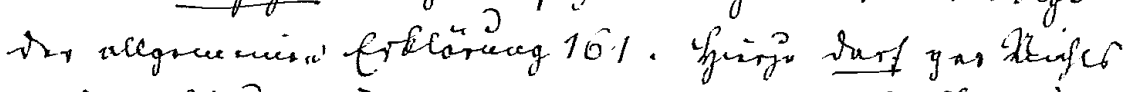

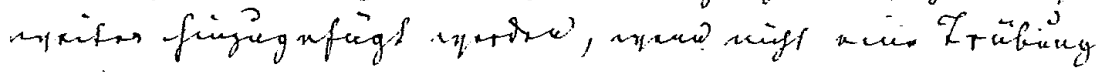
mic frowew foce'.

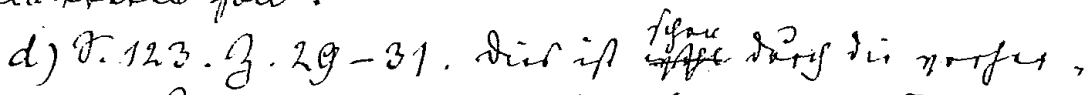

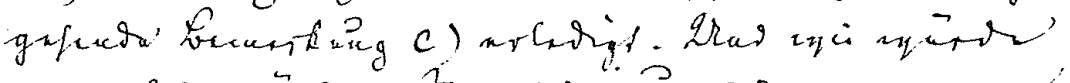

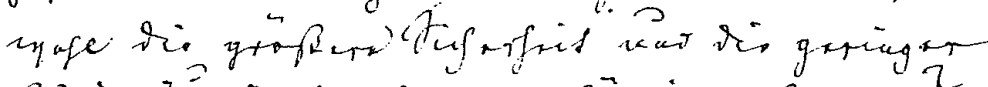

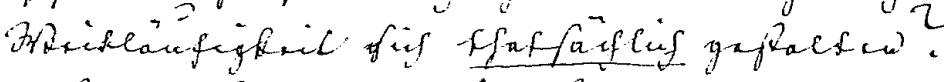

$2 \pi$

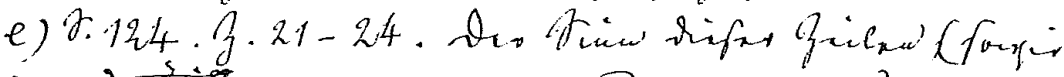

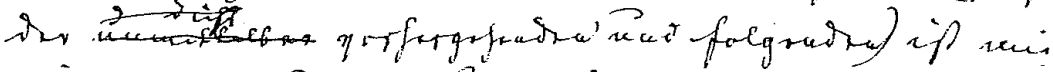

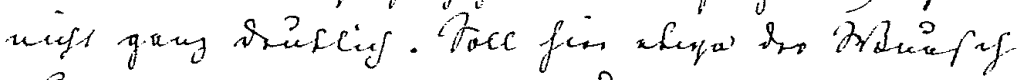

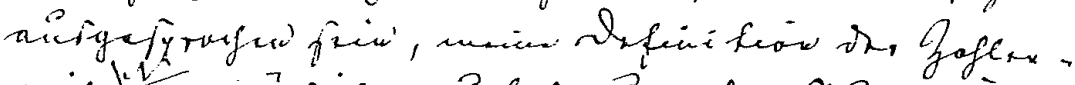

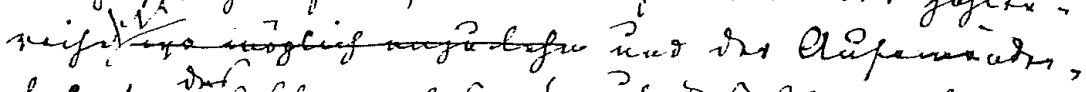

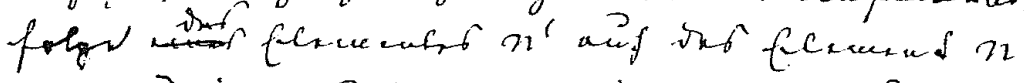

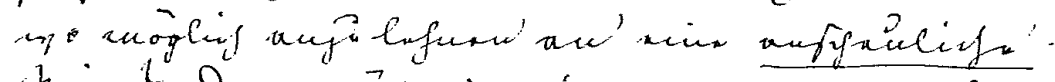

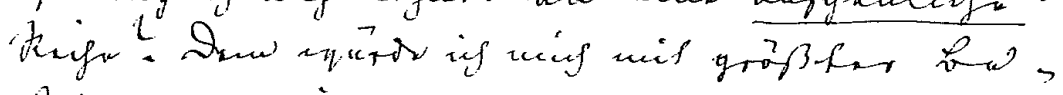

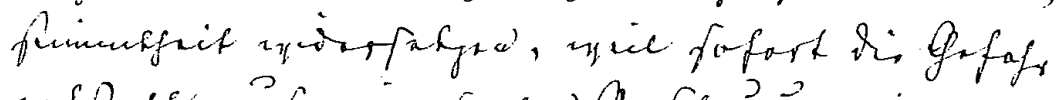

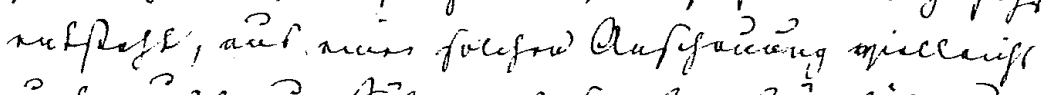

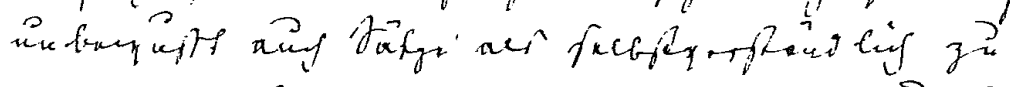

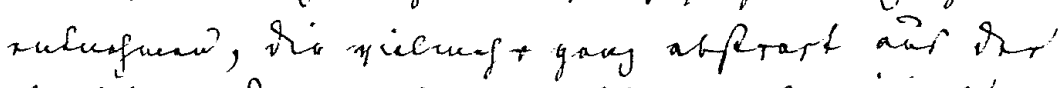

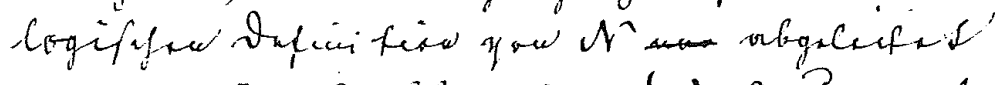

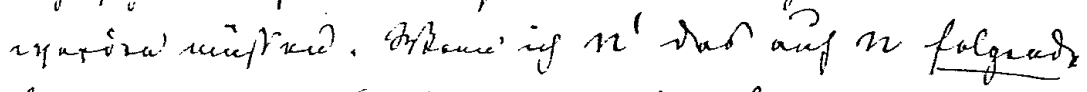

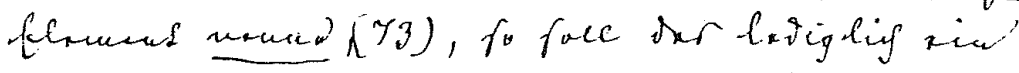


10.

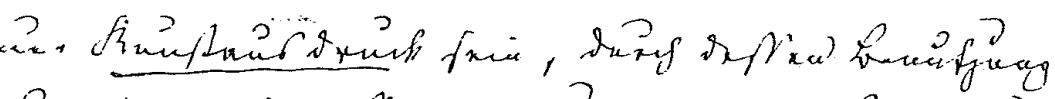

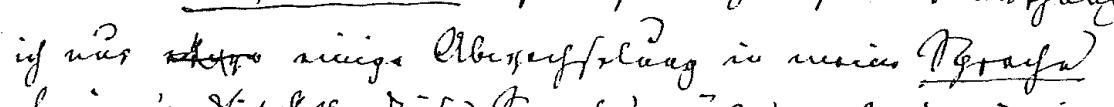

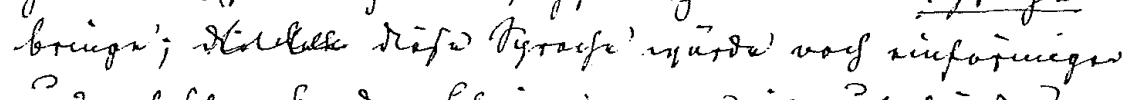

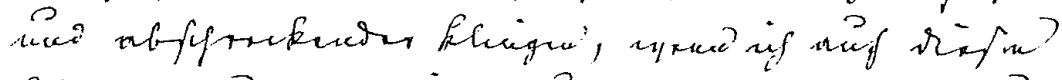

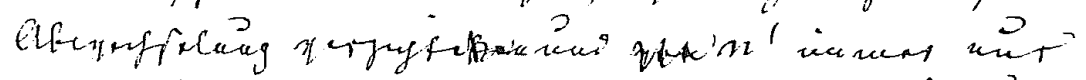

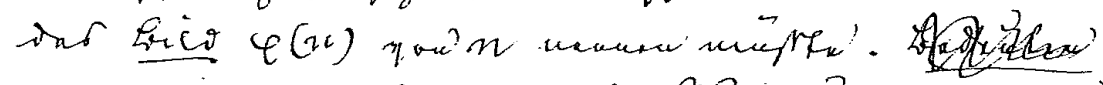

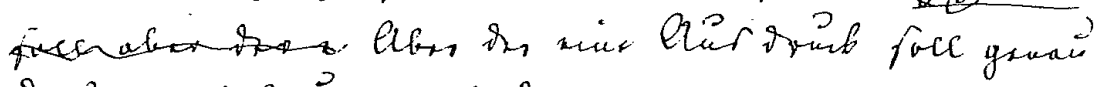

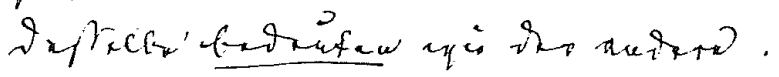

f) $9.124 \cdot 3 \cdot 33-7.125 \cdot 3 \cdot 7 \cdot$. Oar in so

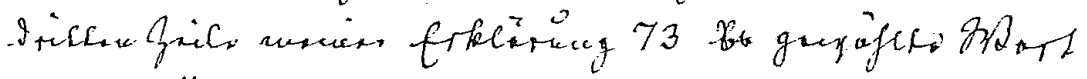

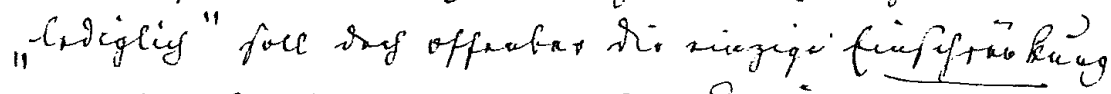

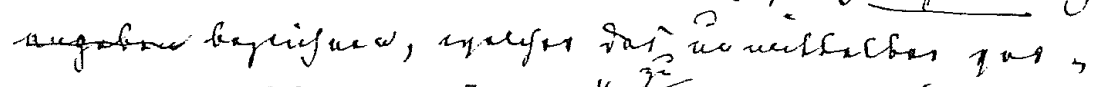

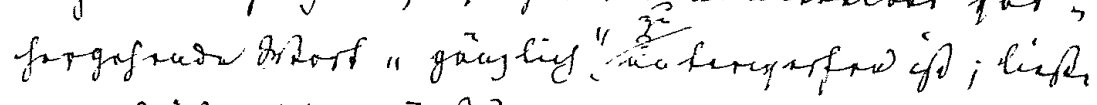

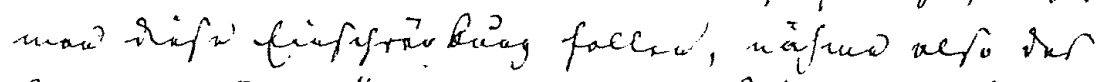

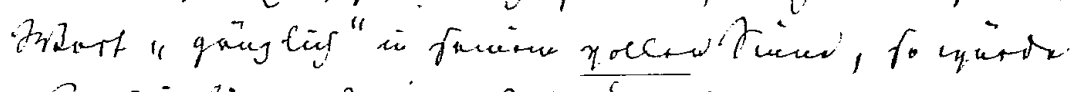

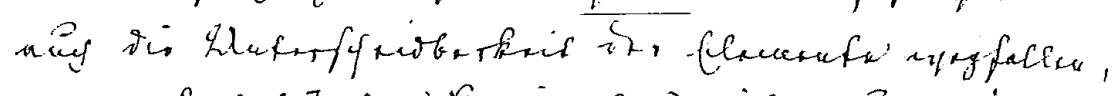

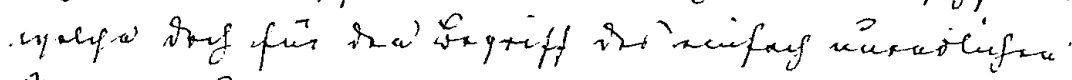

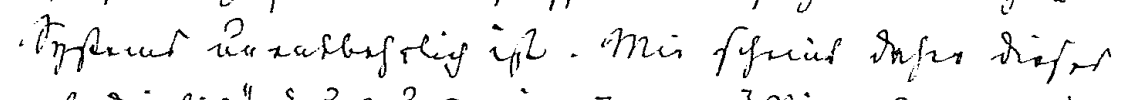

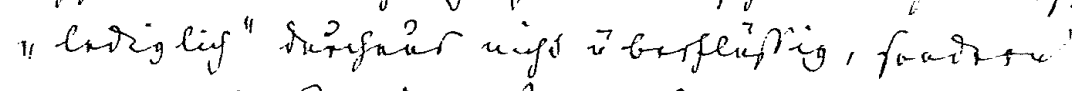

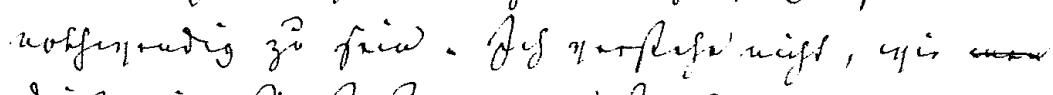

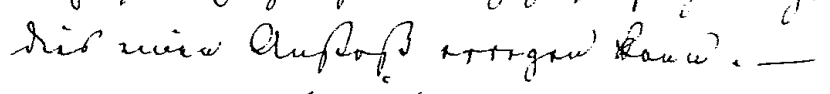

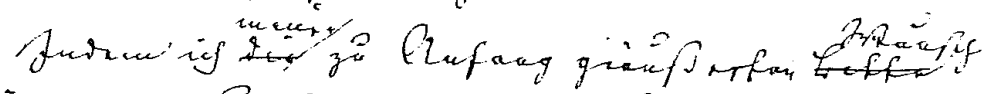

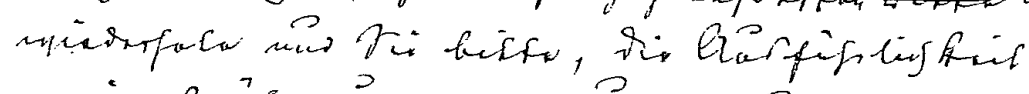

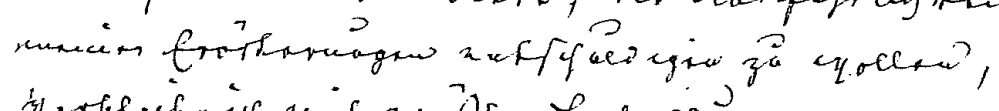

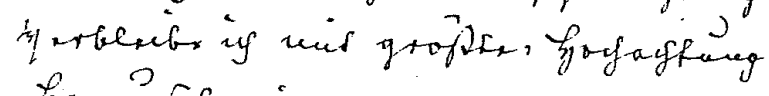

trackifaterio,

27. Frbruar 18g0.
Aft

xigatreftar

tr. Patring: 


\section{Dedekinds Zahlenschrift - Eckstein und Stein des Anstoßes.}

2.1. Das Thema hat einen historischen Aspekt und einen theoretischzukunftsweisenden. Die Erörterung des ersteren wird zeigen, daß die von Dedekind vorgezeichneten Wege immer noch darauf warten, endlich wieder begangen zu werden, d.h. der zweite Aspekt ist so aktuell wie eh und je ${ }^{9}$.

„Das Unendliche” als begriffliches Problem, von Dedekind vor über 120 Jahren ein für alle Mal erledigt, geistert immer noch durch die Literatur, und Dedekinds „Zahlenschrift” (von ihm so genannt) wird gerne etwas scheel angesehen, noch lieber schlicht ignoriert, jedenfalls de facto, an äußerlicher Würdigung fehlt es nicht.

Das hat inhaltliche und andere Gründe. Kein mathematischer Begriff ist so tief und vielfältig in unserem Denken verwurzelt wie der einer natürlichen Zahl, und das von Kindesbeinen an. Kroneckers berühmten Ausspruch Die ganzen Zahlen hat der liebe Gott gemacht, alles andere ist Menschenwerk ${ }^{10}$ kann man daher gut verstehen.

Weniger gut kann ich Hilbert verstehen, dessen Name wie kein anderer mit dem axiomatischen Denken assoziiert wird (Man muß jederzeit an Stelle von Punkten, Geraden, Ebenen auch Tische, Stühle, Bierseidel sagen können $\left.{ }^{11}\right)$, und der im Gegensatz zu Kronecker die aufkommende Mengenlehre und auch Cantor sowie Zermelo persönlich nach Kräften gefördert hat. 1925, also fast 40 Jahre nach Dedekinds Zahlenschrift, stellt er das Problem des Unendlichen im Sinne der unendlichen Gesamtheit vor (ohne Dedekinds Definition auch nur zu erwähnen) und beschreibt seine Klärungsversuche [11] (natürlich mittels Beweistheorie). Dedekind wird folgendermaßen erwähnt:

Schon die beiden um die Grundlagen der Mathematik hochverdienten Mathematiker Frege und Dedekind haben - unabhängig voneinander — das aktual Unendliche angewandt und zwar zu dem Zwecke, die Arithmetik unabhängig von aller Anschaung und Erfahrung auf reine Logik zu begründen und durch diese allein zu deduzieren. Dedekinds Bestreben ging sogar soweit, die endliche Anzahl nicht der Anschaung zu entnehmen, sondern unter wesentlicher Benutzung des Begriffes der unendlichen Mengen rein logisch abzuleiten.

\footnotetext{
${ }^{9}$ Aus dem Nachwort der Herausgeber von Dedekinds Ges. Werken (1932):

Es ist ein Zeichen, wie Dedekind seiner Zeit voraus war, daß seine Werke noch heute lebendig sind, ja daß sie vielleicht erst heute ganz lebendig geworden sind.

${ }^{10}$ Laut H. Webers Nachruf [Jahrber. DMV 2 (1892)] auf der Berliner Naturforscherversammlung 1886 getan, also bevor Dedekinds Zahlenschrift erschienen war.

${ }^{11}$ Vergleiche mit folgendem, sich ebenfalls auf die euklidische Geometrie beziehenden Passus aus Dedekinds Brief vom 27. Juli 1876 an R. Lipschitz [Ges. Werke III, 479]:

Eine untrügliche Methode einer solchen Analyse besteht für mich darin, alle Kunstausdrücke durch beliebige neu erfundene (bisher sinnlose) Worte zu ersetzen, das Gebäude darf, wenn es richtig construirt ist, dadurch nicht einstürzen, und ich behaupte z.B., daß meine Theorie der reellen Zahlen diese Probe aushält.
} 
Insbesondere war es ein von Zermelo und Russell gefundener Widerspruch, dessen Bekanntwerden in der mathematischen Welt geradezu von katastrophaler Wirkung war. Angesichts dieser Paradoxien gaben Frege und Dedekind ihren Standpunkt auf und räumten das Feld: Dedekind trug lange Bedenken, von seiner epochemachenden Abhandlung "Was sind und was sollen die Zahlen" eine Neuauflage zuzulassen; und auch Frege... .

Schon Kant hat gelehrt - und zwar bildet dies einen integrierenden Bestandteil seiner Lehre -, daß die Mathematik über einen unabhängig von aller Logik gesicherten Inhalt verfügt und daher nie und nimmer allein durch Logik begründet werden kann, weshalb auch die Bestrebungen von Frege und Dedekind scheitern mußten.

Wenn schon Kant, dann auch Bolzano [4], sei es auch nur der „selbstdenkenden Köpfe in Deutschland" wegen:

Ich meines Teils will nur gleich offenherzig bekennen, daß ich mich bis zur Stunde - wie von der Wahrheit so mancher anderen Lehren der kritischen Philosophie - so insbesondere auch von der Richtigkeit der Kantischen Behauptungen über die reinen Anschauungen und über das Konstruieren der Begriffe durch sie, nicht habe überzeugen können. Ich glaube noch immer, daß schon in dem Begriffe einer reinen (d.h. apriorischen) Anschauung ein innerer Widerspruch liege; und noch weit weniger kann ich mich überreden, daß der Begriff der Zahl notwendig in der Zeit konstruiert werden müsse, und daß sonach die Anschauung der Zeit zur Arithmetik wesentlich gehöre. Da ich im Anhange zu dieser Abhandlung mehreres hierüber sage, so begnüge ich mich, hier nur hinzuzufügen, daß es der selbstdenkenden Köpfe in Deutschland noch manche und noch gar viele gibt, welche mit diesen Behauptungen Kants ebensowenig einverstanden sind wie ich.

Lassen wir noch Dedekind selbst zu Worte kommen [Vorwort zu der von Hilbert angesprochenen dritten Auflage (1912)]. Nach das Feld räumen hört sich das jedenfalls nicht an:

Als ich vor etwa acht Jahren aufgefordert wurde, die damals schon vergriffene zweite Auflage dieser Schrift durch eine dritte zu ersetzen, trug ich Bedenken, darauf einzugehen, weil inzwischen sich Zweifel an der Sicherheit wichtiger Grundlagen meiner Auffassung geltend gemacht hatten. Die Bedeutung und teilweise Berechtigung dieser Zweifel verkenne ich auch heute nicht. Aber mein Vertrauen in die innere Harmonie unserer Logik ist dadurch nicht erschüttert; ich glaube, daß eine strenge Untersuchung der Schöpferkraft des Geistes, aus bestimmten Elementen ein neues Bestimmtes, ihr System zu erschaffen, das notwendig von jedem dieser Elemente verschieden ist, gewiß dazu führen wird, die Grundlagen meiner Schrift einwandfrei zu gestalten.

Das Schlüsselwort heißt also Analyse, wie im Kefersteinbrief. 
2.2. Hilbert scheint den Gebrauch des aktual Unendlichen, d. h. unendlicher Mengen, wegen der Antinomien als anrüchig zu empfinden. In [10] jedoch (also drei Jahre zuvor), wo weniger gegen Dedekind als gegen Weyl, Brouwer und den "Verbotsdiktator" Kronecker Front gemacht wird, äußert er sich so:

Und die Paradoxien der Mengenlehre können nicht als Beweis dafür angesehen werden, daß der Begriff der Menge von ganzen Zahlen zu Widersprüchen führt. Im Gegenteil: alle unsere mathematischen Erfahrungen sprechen für die Korrektheit und Widerspruchsfreiheit dieses Begriffes.

Gegen Dedekind bleibt nur ein Vorwurf: Sein klassischer Irrtum bestand darin, daß er das System aller Dinge als Ausgang nahm.

Dedekind ging es allein darum, die Existenz einer unendlichen Menge nachzuweisen; wenn diese nun einfach vorausgesetzt wird, dann müßte die ganze Sache doch eigentlich ganz im Sinne Hilberts sein. ${ }^{12}$

Er versucht aber sein Glück auf einem anderen Wege, nach dem Motto Am Anfang ist das Zeichen. Der Spott läßt nicht lange auf sich warten [16]:

Ist das eine Grundlage für Zahlentheorie? Zweifellos nicht. Man erhält so, wenn man die nötige Phantasie hat, hübsche Zierleisten oder Tapetenborten und für jede eine Fabrikmarke, aber keine Mathematik.

In seiner Entgegnung [2] belehrt Bernays den Spötter über den Unterschied zwischen Figur und Gestalt. Wie er in [1] schreibt, schließt Hilbert, der dies auch selbst kundgibt [12], sich später Kroneckers Auffassung an, d. h. er überläßt die Sache mit den ganzen Zahlen doch lieber dem lieben Gott.

Mit Kronecker ist es umgekehrt: Kaum ist sein großes Wort ausgesprochen, überkommt ihn schon die Lust, dem lieben Gott ein wenig nachzuhelfen [15], und zwar unter Voraussetzung der Ordinalzahlen: In diesen besitzen wir einen Vorrath gewisser, nach einer festen Reihenfolge geordneter Bezeichnungen, welche wir einer Schaar verschiedener und zugleich für uns unterscheidbarer Objecte beilegen können.

2.3. In welchem Sinne hat Dedekind eigentlich das Unendliche als begriffliches Problem erledigt? In haargenau demselben Sinne wie auch Konvergenz erledigt wurde, also durch eine explizite mathematische Definition.

Eine solche Definition, die ein bis dato eigenständiges Element mathematischen Denkens eliminieren soll, ist immer mit einer gewissen Willkür behaftet. Ihre Natürlichkeit bedarf „einer vorausgehenden Analyse“ der relavanten mathematischen Natur „so wie diese sich, gewissermaßen erfahrungsmäßig, unserer Betrachtung darbietet" [§1].

\footnotetext{
${ }^{12}$ Ohne den anschaulichen Endlichkeitsbegriff wäre aber Hilberts finitem Standpunkt und überhaupt seiner ganzen Beweistheorie der Boden entzogen.
} 
Idealerweise führt diese Analyse zu einer Charakterisierung des in Frage stehenden Begriffes, die dann einfach Definition genannt wird. Stattdessen könnte man auch vereinbaren, alles etwa mit Unendlichkeit oder Konvergenz verbundene zu vergessen bis auf eben jene Charakterisierung.

Während der moderne Konvergenzbegriff längst täglich Brot für jeden Studenten ist - ohne ihn wäre die höhere Analysis ein Wühlen im Schlamm fehlt der erzieherische Druck beim (Un)Endlichen: alles vertraute läßt sich aus Dedekinds Definition ableiten.

Analyse - Vergessen - Synthese, dieser Dreiklang ist für mich die Essenz einer inhaltlich verstandenen Grundlagentheorie der Mathematik.

Dedekind drückt es so aus: Was beweisbar ist, soll in der Wissenschaft nicht ohne Beweis geglaubt werden [7], und Hilbert spricht von der Tieferlegung der Fundamente [Axiomatisches Denken. Math. Ann. 78, 405-415 (1918)].

Erst seziert man, dann konstruiert man, so beschreibt Egon Friedell in seiner Kulturgeschichte der Neuzeit [Verlag C. H. Beck, München 1927/2012] die Methode Descartes'.

2.4. Was sollte man natürlicherweise unter dem Begriff Grundlagen der Mathematik verstehen, und was dabei unter Mathematik?

Unter Mathematik verstehe ich (hier) das, was ich in meinem akademischen Leben als Mathematik kennengelernt habe: Sie hat Mengen und Abbildungen im Sinne Dedekinds und Cantors (cum grano salis ${ }^{13}$ ) zum Gegenstand, genauer Aussagen über Mengen und Abbildungen, und stellt die Aufgabe, aus solchen Aussagen weitere Aussagen zu gewinnen. ${ }^{14}$ Wir könnten diese real existierende Mathematik auch Cantorsche Mathematik oder (nach Hilbert) Cantors Paradies nennen. $\mathrm{Zu}$ den Grundlagen der Mathematik, den Elementen mathematischen Denkens, führt die Analyse eines ziemlich beliebigen Stücks Mathematik (Beweis eines halbwegs anspruchsvollen Satzes, vergleichbar mit etwas Erde in der Hand des Chemikers) durch fortwährendes Fragen Was ist das?, Warum gilt das?. In begrifflicher Hinsicht führt das zu folgenden Fragen:

Was ist eine Menge?

Was ist eine Abbildung?

Was ist eine Relation?

Was ist Gleichheit?

Was ist ein Paar (und eine Folge)?

Was ist eine Eigenschaft (etwa der Elemente einer Menge)?

Was ist Existenz?

\footnotetext{
${ }^{13}$ Unter dem Einfluß der Antinomien sagt man gelegentlich Klasse statt Menge.

${ }^{14}$ Kaplanski: Our business is theorems.
} 
In der Mathematik wird so oder so die Existenz einer unendlichen Menge vorausgesetzt, und damit erledigt sich fürs erste der Themenkreis Zahlen und (Un)Endlichkeit. Ihn aus dem Dunst des Gottgegebenen, Evidenten, Anschaulichen herausgelöst und Cantors Paradies einverleibt zu haben, ist das Hauptverdienst von Dedekinds Zahlenschrift(en).

Ich möchte mich mit obigen Fragen im Geiste Dedekinds befassen, wie er in seiner Zahlenschrift und im Kefersteinbrief zum Ausdruck kommt. Dabei sind vor allem die naiv-anschaulichen Begriffe endlich, natürliche Zahl, Folge als vergessen anzusehen, und diese Prämisse macht die im 20. Jahrhundert entstandenen, unter dem terminus technicus Grundlagen firmierenden Theorien hinfällig (Mathematische Logik, Beweistheorie, axiomatische Mengenlehre ${ }^{15}$ ). Mit ihnen setzte sich schon vor gut 50 Jahren ein „selbstdenkender Kopf“ auseinander: Bernays' Schüler Wittenberg, in seinem Buche [18].

S. 17: Es waren vor etwa fünfzig Jahren in den Grundlagen der Mathematik Schwierigkeiten aufgetaucht, die für die Mathematiker ein eigentliches Schockerlebnis darstellten und den Ausgangspunkt für die moderne mathematische Grundlagenforschung abgaben, ohne daß diese doch - bei aller Bedeutsamkeit ihrer Ergebnisse - jene Schwierigkeiten in befriedigender Weise zu beseitigen vermocht hätte. Den Schlüssel zu der vorliegenden Arbeit bildete nun die Einsicht, dass als das eigentliche durch jene Schwierigkeiten aufgeworfene Problem das Problem der Kriterie $n^{16}$ angesehen werden muß, unter denen zu mathematischen Grundlagenfragen Stellung zu nehmen ist. In der Tat entstanden im Gefolge jener Schwierigkeiten Meinungsverschiedenheiten darüber, was in der mathematischen Begriffsbildung und im mathematischen Schließen als «zulässig» angesehen werden soll und was nicht; die sich daraus ergebenden Diskussionen zeichneten sich durch hochgradige Willkür aus; sie stellten dogmatische und stark subjektive Auseinandersetzungen zwischen einzelnen Mathematikerpersönlichkeiten dar, ohne dass klar würde, welches die objektiven und zwingenden Kriterien seien, denen sich jene Meinungsverschiedenheiten unterzuordnen hätten.

S. 293: so ergibt sich, dass im Endeffekt der Formalismus im wesentlichen lediglich neuartige mathematische Disziplinen erschlossen hat, die als solche interessant und vielversprechend sind, die aber mit der ursprünglichen Aufgabestellung einer Klärung der Verhältnisse in den Grundlagen der Mathematik nicht mehr sehr viel zu tun haben.

\footnotetext{
${ }^{15}$ Gemeint ist ihre postzermelosche Ausprägung. Nicht nur in [20], auch noch in [21] liegt Zermelo auf der Linie Dedekinds; so wirft er Fraenkel vor, er verfahre konstruktiv, was dem Zweck und Wesen der axiomatischen Methode im Grunde widerspricht und außerdem vom Begriffe der endlichen Anzahl abhängt, dessen Klärung doch gerade eine der Hauptaufgaben der Mengenlehre sein sollte.

${ }^{16} \mathrm{Im}$ Originaltext kursiv.
} 
2.5. Das gesamte Grundlagenthema auf den Punkt gebracht hat schon Augustinus von Hippo (354-430): Was ist also die Zeit? Wenn mich das niemand fragt, weiß ich es. Wenn ich es aber einem erklären möchte, der mich fragt, weiß ich es nicht. So ähnlich hat einmal ein Kollege den Versuch eines Prüflings abgebrochen, den Begriff einer Funktion zu erklären: Man kann es nicht definieren, man sieht sich gegenseitig fest in die Augen und ist sich einig, daß man darunter dasselbe versteht. Aus dem mit der Erkenntnis des Nichtwissens hinsichtlich reeller Zahlen verbundenen Unbehagen, dem Zwang, sich auf geometrische Evidenzen und Anschauungen berufen zu müssen, erwuchs Dedekinds Stetigkeit und irrationale Zahlen [6]:

Für mich war damals das Gefühl der Unbefriedigung ein so überwältigendes, daß ich den festen Entschluß faßte, so lange nachzudenken, bis ich eine rein arithmetische und völlig strenge Begründung der Prinzipien der Infinitesimalanalysis gefunden haben würde.

Und sie wurden gewahr, daß sie nackt waren. Daran fühlen wir uns erinnert, und damit an Hilberts Botschaft Aus dem Paradies, das Cantor uns geschaffen, soll uns niemand vertreiben können [9]. Wie dies zu bewerkstelligen sei, deutet Heinrich von Kleist in seiner Erzählung Über das Marionettentheater an:

Mithin, sagte ich ein wenig zerstreut, müßten wir wieder von dem Baum der Erkenntnis essen, um in den Stand der Unschuld zurückzufallen? Allerdings, antwortete er, das ist das letzte Kapitel von der Geschichte der Welt.

Salopp ausgedrückt: Zurück zur Natur? Ja, also volle Kraft voraus!

2.6. Wo Licht ist, ist auch Schatten, und aus Fehlern und Schwächen kann man lernen. Was also gibt es an Dedekind auszusetzen? Beginnen wir mit seinem „klassischen Fehler", das „System $S$ aller Dinge“ vorauszusetzen (und übergehen wie Hilbert seine tatsächliche, seine Gedankenwelt und sein eigenes Ich involvierende Argumentation mit freundlichem Schweigen).

Daß $S$ zu einem Widerspruch führt, wie auch die hypothetische Menge $\mathcal{M}$ aller Mengen, folgt sofort aus Cantors Satz, daß die Potenzmenge $\mathcal{P}(M)$ einer Menge $M$ mächtiger ist als $M$. Er wird bewiesen, indem man zu einem angenommenen injektiven $\alpha: \mathcal{P}(M) \rightarrow M$ die Menge $R$ aller $x \in \operatorname{Bild}(\alpha)$ mit $x \notin x \alpha^{-1}$ betrachtet. Für $M=S$ und $\alpha=\operatorname{id}_{\mathcal{P}(\mathrm{M})}$ ergibt sich die Russellsche Menge $R$ aller Mengen, die sich nicht als Element enthalten, und $\mathcal{M}$ anstelle $S$ führt zu der Menge $R^{*}$ aller Mengen von Mengen, die sich nicht als Element enthalten. Sie führt wie $R$ zu einem Widerspruch. ${ }^{17}$

Das ist der ernste Hintergrund der auf den ersten Blick etwas exotisch anmutenden Russellschen Antinomie; denn der Grundgedanke hinter dem Mengenbegrifff besteht doch darin, mathematisch interessante gleichartige Dinge in einer Menge zu vereinen, warum also nicht die Mengen selbst?

${ }^{17}$ Es gibt einen kleinen Unterschied, siehe 3.2. 
Und wenn $\mathcal{M}$ wie $S$ nicht existiert, weshalb existiert dann etwa die Menge $\{2,3\}$ oder $\{5\}$, oder die leere Menge, das leere „System“, welches Dedekind übrigens aus gewissen Gründen hier ganz ausschließen will, obwohl es für andere Untersuchungen bequem sein kann, ein solches zu erdichten [7].

Die Existenz eines mathematischen Objektes - vorrangig einer Menge oder einer Abbildung - kann nicht von der Dichtkunst eines Menschen abhängen, der geistigen Tätigkeit des Zusammenfassens und Zuordnens.

Nein! Das beharrliche kindliche Fragen Warum?, d. h. die von Dedekind angemahnte strenge Untersuchung der Schöpferkraft des Geistes, ... [2.1], fördert folgendes Prinzip ans Tageslicht, das für einen Mathematiker eigentlich eine Selbstverständlichkeit sein sollte:

Die Existenz eines mathematischen Objektes muß vorausgesetzt oder bewiesen werden, tertium non datur!

Auf dieser Basis können $S$ und $\mathcal{M}$ dann fröhliche und durch Widerspruchsgeister a priori ungefährdete Auferstehung feiern, per Voraussetzung natürlich. Daneben existiert dann aber erstmal nichts.

Wittenberg [S. 69]: Ein Widerspruch ist das Alarmsignal, welches anzeigt, dass man den Weg der Wahrheit verlassen hat.

So wie Schmerzen im menschlichen Organismus körperliche Mängel signalisieren, weisen die Antinomien auf Mängel in unserem mathematischen Denken hin. Und es ist ein bekanntes medizinisches Phänomen, daß das Verlangen nach Schmerzminderung zu einem körperlichen Fehlverhalten führen kann, und daß deshalb (zwecks Vermeidung von Folgeschäden) manchmal Betäubungsmittel verabreicht werden.

Das erinnert zum einen an die r.e. M.: sie ignoriert de facto die Antinomien und bleibt dadurch natürlich; zum andern an Dedekinds Verlangen [2.1], daß ein System notwendig von jedem seiner Elemente verschieden sei; denn ohne die Russellsche Antinomie käme kein Mathematiker auf die Idee, letzteres zu thematisieren, weil es einfach nicht die geringste mathematische Bedeutung hat.

Mit der unausweichlichen Konsequenz, die Existenz einer unendlichen Menge einfach vorauszusetzen, hätte Dedekind sich wohl nicht so leicht abgefunden: Ohne den logischen Existenzbeweis würde es immer zweifelhaft bleiben, ob nicht der Begriff eines solchen Systems vielleicht innere Widersprüche enthält.

Na und? Absolute Sicherheit gibt es nirgends, daran ändert auch der schönste Widerspruchsfreiheitsbeweis nichts, und es gibt keine voraussetzungslose Mathematik. Lies Dedekinds Motto [2.3] daher mit folgendem Zusatz: Und was nicht beweisbar ist, sollte (soweit benötigt) explizit vorausgesetzt werden. 
In einer inhaltlichen Grundlagentheorie geht es also darum, durch Vergessen das einfach geglaubte, inbesondere den Bestand an augustinischen (inhaltlichen) Begriffen, auf seinen innersten Kern zu reduzieren, und dann über geeignete Voraussetzungen das Vergessene so weit wie möglich zu rekonstruieren.

Ein externer unverbildeter Beobachter der mathematischen Szene, der einfach das Tun der Mathematiker studiert (vielleicht um selber einer zu werden ${ }^{18}$ ) vermag nichts anderes wahrzunehmen als Kommunikation. Daher die Erwartung, daß nach allem Vergessen und Eliminieren ein rein sprachliches allertiefstes Fundament erscheinen wird.

In seiner Grundbedeutung zeigt ein Zeichen etwas, es informiert, teilt mit, gibt ein Wissen weiter oder eine Weisung (Wegweisung, Beweis).

Das rückt Hilberts Am Anfang ist das Zeichen in die Nähe des biblischen Im Anfang war das Wort. Schließen möchte ich aber mit einem anderen Bibelwort:

Der Stein, den die Bauleute verworfen haben, ist zum Eckstein geworden.

\section{Was ist Gleichheit?}

3.1. In der Mathematik wird Gleichheit durchweg als Identität verstanden, ein Begriff, der einer rationalen Beschreibung nicht fähig ist und daher auch philosophisch-logische Gleichheit genannt wird, ${ }^{19}$ ein augustinischer Begriff also, zugleich eine Hauptkomponente des paradiesischen an Cantors Paradies. Mit ihm Hand in Hand geht ein naiv-gegenständlicher Objektbegriff.

In Anfängervorlesungen pflegen wir aber die „Gleichheit" von Mengen (und Abbildungen) zu definieren, und können nicht mehr als Reflexivität, Symmetrie und Transitivität nachweisen. Gleichzeitig wird den Studenten eingehämmert, daß sie in eine Definition nicht mehr hineininterpretieren dürfen als logisch aus ihr abgeleitet werden kann, ganz gleichgültig, welche Assoziationen die verwendeten Worte und Symbole (hier gleich und $=$ ) hervorrufen (do what I say, not what I do). Folgende Standpunkte bieten sich an:

1) Gleichheit (= Identität) wird als gottgegeben voraus- und die von Mengen wie von Abbildungen axiomatisch festgesetzt.

Aus „Definitionen“ werden also Voraussetzungen.

2) Gleichheit wird generell nur als Äquivalenzrelation verstanden.

2a) Die Gleichheit von Elementen einer Menge, oder sogar die von beliebigen Dingen, wird ganz allgemein irgendwie definiert.

2b) Die Gleichheitsbeziehung auf einer Menge $M$ gehört genau so zu $M$ wie zu einer Gruppe $G$ eine Multiplikation. ${ }^{20}$ Die Voraussetzung „M Menge“ schließt ein, daß auf $M$ eine Äquivalenzrelation $=\left(\right.$ oder $\left.={ }_{M}\right)$ gegeben ist.

\footnotetext{
${ }^{18}$ So wie theoretisch ein „guter" Prediger oder Politiker werden kann, wer den richtigen Gebrauch gewisser Worte lernt (ohne deren Inhalt zu kennen).

${ }^{19}$ Denn wo die Begriffe fehlen ...

${ }^{20}$ Der Grundmenge von $G$ entspricht bei dieser Analogie der Grundbereich von $M$.
} 
Wie die einer jeden mathematischen Theorie, so sind auch die Grundbegriffe der Mathematik insgesamt so zu gestalten, daß sie gut miteinander harmonieren und flexibel sind, mit guten Vererbungseigenschaften, ohne irrelevanten Ballast. Dazu ein schönes Zitat aus dem Buche Physik der Gegenwart (Athenäum Verlag, Bonn 1952) von C. F. v. Weizsäcker und J. Juilfs:

Ein physikalisches Gesetz beweist sich wie ein Licht, das in einem dunklen Raum aufgeht, durch die Ordnung, die es sichtbar macht.

Auch unter den früher erläuterten Prinzipien ist $2 \mathrm{~b}$ die kanonische Option, weil sie mit einem Minimum an Information verbunden ist, mit einem Maximum an Vergessen. Das soll uns aber nicht davon abhalten, auch andere Ansichten zu erörtern.

3.2. De jure scheint Dedekind die Gleichheit (Identität) von „Dingen“ auf das Leibnizsche Eadem sunt quae sibi ubique substitui possunt, salva veritate zu gründen:

Ein Ding a ist dasselbe wie $b$ (identisch mit $b$ ), und $b$ dasselbe wie $a$, wenn alles was von a gedacht werden kann, auch von $b$, und wenn alles, was von $b$ gilt, auch von a gedacht werden kann.

Gemeint ist wohl: Was für $a$ gilt, gilt auch für $b$, und umgekehrt. Als angebliche Konsequenz aus diesem Gleichheitskriterium für Dinge präsentiert er das Kriterium

(*) $\quad x \in A \Longleftrightarrow x \in B$

für die Gleichheit von Mengen $A, B$. Er müßte also fähig sein, aus $(*)$ und $A \in A$ dieselbe Eigenschaft $B \in B$ von $B$ herzuleiten, was aber wohl kaum möglich ist.

3.3. Somit ist auch das Thema Gleichheit ein Schwachpunkt in Dedekinds Zahlenschrift. Anzuerkennen ist aber, daß er es überhaupt anspricht und nicht wie die Masse der Mathematiker einfach ignoriert oder mit dem bequemen Attribut logisch abhakt. Auch Zermelo [20] ist es nur eine notationelle Randbemerkung wert:

(A) Sollen zwei Symbole a und b dasselbe Ding bezeichnen, so schreiben wir $a=b$, im entgegengesetzten Falle $a \neq b$.

Das kritisiert Fraenkel [8] in einem schönen Pendant zu Fußnote 15:

Diese dem form al en Charakter der axiomatischen Methode nicht ganz angepaßte Zurückführung der Gleichheit auf die in haltlich geprägte Beziehung der Identität kann natürlich für die Zwecke der Mengenlehre nicht ausreichen. 
Dann weist er auf das gerade eben ausgenutzte Phänomen hin, daß sich nämlich allem Anschein nach aus Zermelos Axiom der Bestimmtheit

(B) $A=B \Longleftrightarrow x \in A \Leftrightarrow x \in B$ (für alle Objekte $x$ )

nicht die duale Regel

(C) $\quad a=b \Longleftrightarrow a \in M \Leftrightarrow b \in M$ (für alle Mengen $M$ )

für Mengen $a, b$ ableiten läßt. Er erwägt die Möglichkeit, $=($ neben $\in)$ als zweite und gleichberechtigte undefinierte Grundbeziehung einzuführen und (C) wie (B) axiomatisch zu fordern (wie in [3]). Das habe aber den Nachteil, das Axiomensystem mit zwe $i$ undefinierten Grundbeziehungen und mit z we $i$ "relationalen" Axiomen (B) und (C) - neben den unverändert bleibenden existenzialen Axiomen $-z u$ belasten. Er schlägt nun die Zusatzannahme ${ }^{21}$

(D) $a \in X \Longrightarrow a$ ist Menge

vor, wie aus anderen Gründen schon in [9], weil dann durch (B), gelesen als Definition, auch die Gleichheitsbeziehung für die Elemente einer Menge definiert wäre. Außerdem wäre $(\mathrm{C})$ zu fordern. ${ }^{22}$

Echte Zusatzannahmen sind das Gegenteil von Vergessen. Sie laufen auf eine Höherlegung der Fundamente statt einer Tieferlegung hinaus.

Zermelo [19]: In der Tat müssen die Prinzipien aus der Wissenschaft, nicht die Wissenschaft aus ein für allemal feststehenden Prinzipien beurteilt werden.

Sein Auswahlaxiom bringt in der Tat nur ein bis dato unbewußt angewandtes Denkgesetz ans Tageslicht, es ist keine echte Zusatzannahme.

Auch (C) entspricht nicht ganz der mathematischen Natur: Nirgends in der Mathematik spielen die ein Objekt $a$ enthaltenden Mengen $M$ irgendeine Rolle, außer es handelt sich um Teilmengen einer gegebenen Menge mit Element $a$. Für Elemente $a, b$ und Teilmengen $M$ einer Menge $X$ ist (C) allerdings ein wichtiges Denkgesetz, dem aber nicht mit Brachialgewalt Geltung verschafft werden sollte, sondern über die ohnehin nötige Anpassung des Begriffes Teilmenge an die durch die Abkehr von der Identität geschaffenen neuen Verhältnisse: Eine Teilmenge einer Menge $X$ ist eine Menge $M$ mit

(a) $a \in M \Longrightarrow a \in X$,

(b) $a={ }_{M} b \Longleftrightarrow a={ }_{X} b$ (für $a, b \in M$ ),

(c) $a={ }_{X} b \in M \Longrightarrow a \in M$.

\footnotetext{
${ }^{21}$ Die Tatsache, daß es sich um eine solche handelt, wird in der Literatur oft dadurch verbrämt, ja geradezu in ihr Gegenteil verkehrt, daß man von einem Verzicht auf Urelemente spricht.

${ }^{22}$ Wie Fraenkel bemerkt, kann = genausogut durch $(\mathrm{C})$ definiert werden, dann mit (B) als Axiom. Ganz unabhängig von (D) kann (C), gleichsam eine Mathematisierung der Leibnizregel, auch als Gleichheitsdefinition für allgemeine Objekte dienen. So oder so, es macht nicht den geringsten Unterschied, stattdessen = als ,gleichberechtigte undefinierte Grundbeziehung“" einzuführen und dann mittels (B)(C) festzunageln.
} 
Dadurch ändert sich in der mathematischen Praxis nichts:

Die Frage, ob eine Menge $M$ Teilmenge einer Menge $N$ ist, stellt sich nur, wenn $M$ und $N$ Teilmengen einer Menge $X$ sind, und dann genügt $a \in M \Rightarrow$ $a \in N$. Außerden haben die natürlicherweise vorkommenden Teilmengen (im alten Sinne) immer auch die Eigenschaften (b) und (c). ${ }^{23}$

3.4. Immerhin reduziert Fraenkels Vorgehen die Gleichheitsbeziehung auf den Rang einer Äquivalenzrelation, es entspricht 2a. Die damit verbundenen Probleme allerdings scheint er nicht zu sehen. An sie denkt Bernays vielleicht, wenn er sich in [3] zu genau der Ansicht bekennt, die doch laut Fraenkel „natürlich für die Zwecke der Mengenlehre nicht ausreichen kann“.

S. 52: We do not introduce here equality by an explicit definition, because we want to suggest the interpretation of equality as individual identity, whereas by 2.9 or $2^{*}$ taken as definition, equality is introduced only as an equivalence relation.

Pikanterweise ist Fraenkel Mitautor von [3] und zuständig für die historische Einleitung, in der das Thema Gleichheit noch einmal recht ausführlich besprochen wird. Insbesondere listet er die drei möglichen Sichtweisen auf (1, 2a, 2b in 3.1), mit seiner Präferenz für 2a, genau wie oben beschrieben. Bernays bleibt aber Fraenkels Zusatzaxiom (D) treu, und dafür muß dann sogar Dedekind herhalten:

Thus our axiom of extensionality implies the assumption that all elements in our system are themselves sets. This - at the first glance astonishing-feature, common to most newer axiomatic systems of set theory, can be understood as a result of an extension of Dedekind's method of introducing the real numbers, which indeed have the role of elements in analysis, as sets. In view of the program of embracing all classical mathematics in the system of set theory, we are induced to strengthen the said Dedekind procedure to the effect that all mathematical objects become sets, and in fact from this device considerable simplifications are arising.

Dedekind war zwar seiner Zeit weit voraus, aber seine Bezeichnung Körper bezog sich ursprünglich nur auf Teilkörper des komplexen Zahlkörpers.

Immerhin betont er, daß nicht seine „Schnitte“ als reelle Zahlen zu verstehen seien, sondern daß jeder Schnitt eine reelle Zahl „erschaffe“, daß seine so erschaffenen reellen Zahlen den Schnitten nur bijektiv entsprechen. Er vermeidet es also, ganz im Sinne des modernen axiomatischen Standpunkts, seine reellen Zahlen mit irrelevantem Ballast zu belasten.

${ }^{23}$ Die Verträglichkeit mit $=$ ist natürlich grundsätzlich in jedem Einzelfall zu überprüfen, wie auch bei Abbildungen und Relationen. 
3.5. Fraenkels weitere Ausführungen in [3] führen schnell zu den Problemen, die mit der Aufgabe des Identitätsbegriffes verbunden sind. Diese sind ganz unabhägig von (D), gelten also genauso für Cantors Paradies. Betrachten wir etwa die Einführung der Potenzmenge (Axiom IV):

For any set $s$, there exists the set whose elements are all subsets of $s$.

Weiter: This set is called the power-set of s and denoted by $\Pi(s)$.

Die Worte the und this deuten darauf hin, daß es „nur eine“ solche Menge gibt, aber das bedeutet doch nur, daß alle solche Mengen whose elements are all subsets of $s$ äquivalent sind im Sinne der Äquivalenzrelation $=$. Wie also ist es möglich, von $\Pi(s)$ zu reden? Nur auf eine Weise: Indem eine entsprechende Abbildung $\Pi$ - und zwar im ursprünglichen Dedekindschen Sinne - explizit vorausgesetzt wird, d. h. Axiom IV wäre etwa so zu formulieren:

$\Pi$ ist eine Abbildung, die jeder Menge $s$ eine Menge $\Pi(s)$ zuordnet, derart daß die Teilmengen von s die Elemente von $\Pi(s)$ sind.

Ohne (D) ist noch vorauszusetzen, daß die Gleichheitsbeziehung auf $\Pi(s)$ ist wie gewünscht, d.h. wie in (B).

Genauso wäre zu verfahren, um zu jedem $x \in s$ die Teilmenge $\{x\} \mathrm{zu}$ „definieren“. Für Bildungen wie $\{x, y\}$ oder $A \cap B$ scheint man eine Art Funktion von zwei Variablen zu brauchen. Zum Glück genügt aber der gewöhnliche Abbildungsbegriff, und zwar ohne daß Paare zur Verfügung stehen [17]:

Setze, um etwa $A \cap B$ für $A, B \in \Pi(s)$ zu erhalten, eine Abbildung $\cap$ voraus, die jedem $A$ eine Abbildung $A \cap$ zuordnet, die jedem $B$ eine Teilmenge $A \cap B$ mit ... zuordnet. Natürlich soll auch $s$,variabel“ sein. Deshalb brauchen wir hier eigentlich eine Funktion $\cap$ von drei Variablen ${ }^{24}$ : Sie ordnet jedem $s$ eine Funktion $\cap_{s}$ wie eben zu.

Weshalb rede ich hier nur von Teilmengen einer Menge statt von beliebigen Mengen wie Fraenkel, und wie es auch sonst üblich ist? Ich verfahre auch in einer Anfängervorlesung so, aus drei Gründen:

Weil nur Schnitte und Vereinigungen von Teilmengen mathematisch bedeutsam sind, weil der leere Schnitt so keinen Sonderfall mehr darstellt, und weil ich immer die Gleichheitsfrage im Hinterkopf habe. Wenn nämlich nur die Gleichheitsbeziehungen $={ }_{A}$ und $=_{B}$ zur Verfügung stehen, von denen nichts weiter bekannt ist, als daß es sich jeweils um eine Äquivalenzrelation handelt, was soll dann die Gleichheitsbeziehung von $A \cap B$ oder $A \cup B$ sein?

Schon Zermelo [20] verzichtet auf einen eigenständigen Abbildungsbegriff und versteht unter einer Abbildung eine Relation $\alpha$ mit der Eigenschaft, daß zu jedem $x$ (aus dem Definitionsbereich) genau ein $y$ existiert mit $x \alpha y .{ }^{25}$ „Dieses“ $y$ kann aber nur dann einfach mit $\alpha(x)$,,bezeichnet" werden [analog $\Pi(s)$ ], wenn auf dem Wertebereich $Y$ die Identität als Gleichheitsbeziehung vorliegt.

\footnotetext{
${ }^{24}$ Analog für $\{a, b\}$, und für $\{a\}$ eine von zwei.

${ }^{25}$ Tatsächlich scheint er nur injektive Abbildungen zu betrachten (wie auch Cantor?).
} 
Wir sehen: Wer sich nicht mehr auf die Krücke Identität stützen will, kann sich ohne den ursprünglichen Abbildungsbegriff nicht mehr in der gewünschten Allgemeinheit ausdrücken. Und darin sehe ich die tiefere Wahrheit dessen, was Dedekind im Vorwort zu seiner Zahlenschrift [7] ausführt über die Fähigkeit des Geistes, Dinge auf Dinge zu beziehen, einem Dinge ein Ding entsprechen zu lassen, oder ein Ding durch ein Ding abzubilden, ohne welche Fähigkeit überhaupt kein Denken möglich ist.

Damit wäre auch die Frage beantwortet, was die Abbildungen überhaupt „sollen“, über ihre Bedeutung als Forschungsobjekte hinaus. Die analoge Frage „was sollen die Mengen?" beantwortet sich analog: Sie stellen nicht nur ein interessantes und wichtiges Gebiet mathematischer Forschung dar - dafür steht Cantor mit seiner Theorie der Kardinal- und Ordinalzahlen, der Mengenlehre im engeren Sinne -, sondern drängen sich dem in immer größere Allgemeinheiten fortschreitenden mathematischen Forschergeist geradezu auf, als das diesem Fortschritt den Weg bahnende sprachliche Ausdrucksmittel.

In [7] wird zum ersten Mal eine Situation rein mengen,"theoretisch“ - mit explizitem Bezug auf die eigens hierfür eingeführten Begriffe System (= Menge) und Abbildung - vorgestellt und behandelt (darin liegt die eigentliche historische Bedeutung dieses Opus):

So wie später etwa unter einer Gruppe eine Menge $G$ zusammen mit einer Abbildung $\alpha: G \times G \rightarrow G$ einer gewissen Art verstanden wurde, betrachete Dedekind eine Menge $N$ zusammen mit einer Abbildung $\varphi: N \rightarrow N$ einer gewissen Art (und nannte die Elemente von $N$ dann natürliche Zahlen). ${ }^{26}$

Als erste folgten ihm H. Weber (Lehrbuch der Algebra, Vieweg, Braunschweig 1896) und E. Steinitz (Algebraische Theorie der Körper, J. f. Math. (Crelles Journal) 137, 167-309 (1910)), die beide den Körperbegriff in seiner natürlichen Allgemeinheit verwendeten.

3.6. Ein rein mathematischer Gleichheitsbegriff gemäß 2b ist nicht nur mit Problemen verbunden, sondern auch mit Wohltaten. Vor allem mit solchen, die der mathematische Jargon schon vorweggenommen hat: Modulo ... ist ... .

Kern der Sache ist die Möglichkeit, anstelle einer Partition $\mathcal{P}$ einer Menge $M$, mit dazugehöriger Äquivalenzrelation $\rho$, die neue Menge $M / \rho$ zu betrachten; sie hat denselben Grundbereich wie $M(x \in M / \rho \Leftrightarrow x \in M)$, aber $\rho$ anstelle $=$ als Gleichheitsbeziehung. Ein solches $M / \rho$ nenne ich eine Faktormenge von $M$.

Während die mit $\rho$ verträglichen Abbildungen $\alpha: M \rightarrow N$ üblicherweise mit den Abbildungen $\beta: \mathcal{P} \rightarrow N$,identifiziert" werden, sind sie nun genau die Abbildungen von $M / \rho$ in $N$.

\footnotetext{
${ }^{26} \mathrm{Daß}$ er „Axiome“ für die natürlichen Zahlen angegeben habe, ist schlicht Unfug.
} 
Eine mit $\rho$ verträgliche Multiplikation auf $M$ ist auch eine Multiplikation auf $M / \rho$; und mit $M$ ist auch $M / \rho$ eine Gruppe, eine Faktorgruppe von $M \mathrm{im}$ neuen Sinne des Wortes. Und die Homorphismen $M / \rho \rightarrow N$ ( $N$ eine weitere Gruppe) sind einfach die mit $\rho$ verträglichen Homomorphismen $\alpha: M \rightarrow N$.

Unter dem Kern von $\alpha \in \operatorname{Hom}(M, N)$ werde die zu $\alpha$ gehörige Äquivalenzrelation von $M$ verstanden. Der Homorphiesatz lautet dann so:

Ein Homomorphismus $\alpha: M \rightarrow N$ ist ein Isomorphismus von $M / \operatorname{Kern}(\alpha)$ auf das Bild $M \alpha$.

Außerdem ist die Faktorgruppe $M / \rho / \tau$ der Faktorgruppe $M / \rho$ schlicht und einfach die Faktorgruppe $M / \tau$ von $M$. Dabei wie im Homomorphiesatz könnte Gruppe auch einfach Menge mit Multiplikation bedeuten.

\section{Was ist eine Menge?}

4.1. Erstes Ziel ist ein mathematisch adäquates Verständnis dessen, was man anschaulich unter einer Gesamtheit von irgendwelchen Dingen versteht.

Denken wir uns alles vergessen, was mit dem Mengenbegriff der r. e. M. verbunden ist, außer der Möglichkeit, von den Elementen einer Menge zu reden ${ }^{27}$. Was dann übrig bleibt, nenne ich einen Bereich, wie schon in $\S 3$ (Fußnote 20) angedeutet.

Was neben einer Gleichheitsbeziehung noch alles zu dem Begriff einer Menge gehören soll, in der in Aussicht gestellten Synthese, sei zunächst dahingestellt. Ich nenne einen Bereich zusammen mit einer Äquivalenzrelation (etwa =) eine Klasse, was mit dem üblichen Gebrauch dieses Wortes durchaus kompatibel ist.

Das Wort zusammen wird später im Zusammenhang mit der Klärung des Paarbegriffes eliminiert. Was aber ist eine Relation auf einem Bereich A? Ich sehe nur eine Antwort, und diese erfordert den Begriff einer Abbildung auf $A^{28}$ :

Eine Abbildung $\rho$, die jedem $x \in A$ einen Teilbereich $x \rho$ zuordnet.

Die Notation $x \rho y$ für $y \in x \rho$ klärt die Sache.

Der Begriff einer Teilklasse wird gemäß (a)(b)(c) in 3.3 definiert, und Abbildungen zwischen (wie Relationen auf) Klassen sind per definitionem mit den jeweiligen Gleichheitsbeziehungen verträglich. Eine Klassenabbildung $\alpha: A \rightarrow B$ ist also quasi ein Klassenhomomorphismus: $x=y \Rightarrow x \alpha=y \alpha$.

Mit Bereichen läßt sich wenig anfangen, sie dienen nur als Basis für Klassen und damit (später) für Mengen. Weder läßt sich von injektiven oder surjektiven Abbildungen reden, noch von Teilbereichen $\{a, b\}$ oder wenigstens $\{a\}^{29}$ für $a, b \in A$, wohl aber von Inklusion $A \subseteq B$ und Gleichheit $A=B$.

${ }^{27}$ Das ist offenbar der „innerste Kern“ des Mengenbegriffes (jedweder Couleur):

Alles Reden über Mengen involviert den „Begriff“ eines Elementes.

${ }^{28}$ Natürlich im Sinne Dedekinds [7]:

Eine Abbildung $\alpha$ auf $A$ produziert zu jedem Element $x \in A$ ein "Objekt" $x \alpha$.

${ }^{29} \mathrm{Daß}$ ein Bereich „nur“ aus dem Element $a$ besteht, läßt sich nicht formulieren. 
Achtung: Diese Worte und Zeichen haben hier nur notationelle Bedeutung; damit $=$ als Äquivalenzrelation auf dem (vorauszusetzenden) Bereich $\mathcal{B}$ aller Bereiche verstanden werden kann, muß eine solche explizit vorausgesetzt werden, und das erzeugt dann die Klasse $\mathcal{B}$.

Analog läßt sich die Klasse $\mathcal{K}$ aller Klassen in die Welt setzen.

4.2. Was ist ein Element eines Bereiches?

Natürlich ein Objekt (oder Ding), falls dieser Begriff zur Verfügung steht. Die Frage verzweigt sich also so:

(a) Wie sieht die Welt ohne Objekte aus?

(b) Was ist ein Objekt, wenn ES welche GIBT? ${ }^{30}$

Das hängt aufs engste mit dem Begriff einer Abbildung $\alpha$ zusammen. Der Grundgedanke ist doch, daß $\alpha$ jedem Objekt $x$ einer gewissen Art, mit einer gewissen Eigenschaft, ein Objekt $x \alpha$ wiederum einer gewissen Art zuordnet. Das wird in Cantors Paradies dadurch verdeckt, daß man ja einfach die jeweils interessierenden Objekte zu einer Menge (oder Klasse) zusammenfassen kann. Deshalb ist gewöhnlich von einer Abbildung von $A$ in $B$ die Rede, und dafür genügt es, von Elementen von $A$ und $B$ reden zu können, ein Objektbegriff ist a priori nicht erforderlich.

Bei Dedekind [7] ist $\alpha$ nur monogam verheiratet, mit $A$ :

Unter einer Abbildung eines Systems $S$ wird ein Gesetz verstanden, nach welchem zu jedem bestimmten Element $s$ von $S$ ein bestimmtes Ding gehört, welches das Bild von $s$ heißt und mit $\varphi(s)$ bezeichnet wird. ${ }^{31}$

Warum nicht auch noch den so oder so verstandenen Definitionsbereich von $\alpha$ über Bord werfen und so zu einem Maximum an Symmetrie und Einfachheit gelangen? Das Grundwissen über $\alpha$ wäre also:

(*) Wenn $x$ ein Objekt ist, dann ist auch $x \alpha$ ein Objekt.

Dafür und für alles darauf aufbauende ist es gleichgültig, ob Objekt als Grundbegriff vorausgesetzt wird ${ }^{32}$ oder ein Bereich $\mathcal{D}$, dessen Elemente Objekte genannt werden; jeweils mit der Maßgabe, daß alle Abbildungen und Bereiche (auch Klassen) mitsamt ihren Elementen Objekte sind. ${ }^{33}$

\footnotetext{
${ }^{30}$ Wenn ich diesen Begriff vorgebe.

${ }^{31}$ Später betont Dedekind, daß die Bezeichnung $s \varphi$ viel natürlicher sei, und er folgt dieser Einsicht auch schon 1894 [Ges. W. III, 24ff].

${ }^{32}$ Auch dann soll $x \in \mathcal{D}$ für $x$ ist ein Objekt stehen.

${ }^{33}$ Den kundigen Leser erinnert $(*)$ vielleicht an v. Neumanns Axiomatisierung der Mengenlehre [J. f. Math. 125(1925), 219 - 240]:

Wir betrachten zwei Bereiche von Dingen, den der „Argumente“ und den der „Funktionen". Nun ist in diesen Bereichen eine 2-Variablen-Operation [x,y] definiert (lies "Wert der Funktion $x$ für das Argument $y$ “), deren erste Variable $x$ stets "Funktion" und deren zweite Variable y stets „Argument" zu sein hat. Durch sie wird immer ein „Argument" $[x, y]$ gebildet.
} 
Ist $(*)$ mit der r. e. M. kompatibel? Betrachte in dieser eine Abbildung $\alpha$ auf einer Menge $A$. Ein Objekt $x$ liegt dann in $A$ oder es liegt nicht in $A^{34}$. Setze nun $\alpha$ so fort, daß jedem $x$ der zweiten Art irgendein Objekt zugeordnet wird, etwa die leere Menge. Es ist dann egal, ob man mit $\alpha$ oder dieser Fortsetzung arbeitet.

Ein Abbildungsbegriff gemäß (*) ist also zumindest zulässig, und die in 3.1 genannten Prinzipien machen ihn geradezu mandatorisch.

So brauchen wir uns nicht mehr um Definitionsbereiche zu kümmern. Daher läßt sich beispielsweise auch ohne $\mathcal{B}$ von einer Funktion (d. h. Abbildung) reden, die jedem Bereich den Bereich seiner Teilbereiche zuordnet. ${ }^{35}$

Des weiteren werden Einschränkungen auf Teilbereiche begrifflich hinfällig:

Schreibe $\alpha: A \rightarrow B$ wenn $x \alpha \in B$ für alle $x \in A$. Schon Dedekinds Definition impliziert $\alpha: A \rightarrow B^{\prime}$ für jeden Bereich $B^{\prime} \supseteq B$, und jetzt folgt auch die duale Regel $\alpha: A^{\prime} \rightarrow B$ für jedes $A^{\prime} \subseteq A$ (ganz im Sinne der ,inneren Harmonie unserer Logik").

In Worten: Eine Abbildung von $A$ in $B$ ist auch eine Abbildung von jedem Teilbereich von $A$ in jeden Oberbereich von $B$.

Das entsprechende gilt auch für Klassen, wie die folgende Anwendung:

Ist $A^{\prime} \subseteq A$ bezüglich einer Relation $\rho$ von $A$ abgeschlossen, d. $h . x \rho \subseteq A^{\prime}$ für alle $x \in A^{\prime}$, so ist $\rho$ auch eine Relation von $A^{\prime}$.

Insbesondere ist jeder bezüglich $=$ abgeschlossene Teilbereich einer Klasse Grundbereich einer Teilklasse.

Für Bereiche kann man mangels $=($ auf $B)$ überhaupt nicht formulieren, daß $\alpha^{\prime}: A^{\prime} \rightarrow B$ Einschränkung von $\alpha: A \rightarrow B$ ist. Um bei Klassenabbildungen ohne $(*)$ von der Einschränkung $\alpha^{\prime}=\alpha_{\mid A^{\prime}}$ reden zu können, muß eine Funktion von vier Variablen vorausgesetzt werden, ${ }^{36}$ die $A, B, A^{\prime}, \alpha$ ein $\alpha^{\prime}$ zuordnet.

Und Auswahlfunktion müßte als zusätzlicher Grundbegriff eingeführt werden, neben dem einer gewöhnlichen Abbildung $\alpha: A \rightarrow B$ :

Eine Auswahlfunktion $\alpha$ zu $\sigma: A \rightarrow \mathcal{K}$ ( $A$ eine Klasse) ordnet jedem $x \in A$ ein Element $x \alpha$ von $x \sigma$ zu (und ist wie $\sigma$ mit $=$ verträglich).

Weit über die beschriebenen Unbequemlichkeiten hinausgehende Probleme hängen mit der Definition des Klassenbegriffes zusammen [4.4].

Die Diskussion um $(*)$ beantwortet auch (a), letztlich die sich im Anschluß an früheres [3.5] stellende Frage Was sollen die Objekte?:

Die Objekte sind das Schmiermittel für das reibungslose Zusammenwirken von Bereichen und Abbildungen.

\footnotetext{
${ }^{34}$ Das wird in der Literatur nicht selten ausdrücklich von einer Menge verlangt.

${ }^{35}$ Was sollte man in 3.5 denn unter dem Definitionsbereich von $\Pi$ verstehen?

Und was unter dem Wertebereich von $\cap$ ?

${ }^{36}$ Vorher sind die passenden Definitionsbereiche bereitzustellen.
} 
4.3. Was ist ein Paar? Dieser Frage nähern wir uns genau so, wie Dedekind sich einst der Frage Was sind die Zahlen? zuwandte. Seiner Analyse der anschaulichen Zahlenreihe entspricht eine Analyse des vertrauten Produktes $P=A \times B$ von Mengen $A, B$, die das mathematisch wesentliche auf den Tisch legen soll. Das Ergebnis (mit $A_{1}=A$ und $A_{2}=B$, sowie $p_{i}=p \pi_{i}$ für $p \in P$ ):

Zu der Menge $P$ gehören Abbildungen $\pi_{i}: P \rightarrow A_{i}$, sowie eine Funkion $f$ von zwei Variablen, $x \in A$ und $y \in B$, mit Werten in $P$, derart daß

(a) $p=q \Longleftrightarrow p_{i}=q_{i}(i=1,2)$, und

(b) $f(x, y)_{1}=x$ sowie $\left.f(x, y)\right)_{2}=y$.

Erläuterung: $f$ ist im Sinne des Schönfinkelkniffs zu verstehen [3.5], also etwa als Abbildung von $A$ in die Menge $\mathcal{F}(B, P)$ der Abbildungen von $B$ in $P$ : Schreibe $f(x, y)$ statt $y x f$. Nenne $f$ 2-variabel, auch 2-Funktion.

Die zu (b) duale Regel $f\left(p_{1}, p_{2}\right)=p$ folgt aus (a) und (b).

Die auf die Analyse folgende Synthese führt in offensichtlicher Weise zu dem Produkt $P=A \times B$ von Klassen $A, B$ : Setze eine Klasse $P$ voraus plus Abbildungen $\pi_{i}$ und $f$ wie oben, schreibe $(x, y)$ statt $f(x, y)$.

Oft werden Paare $(a, b)$ betrachtet, bei denen $b$ irgendwie von $a$ abhängt. Um ihnen gerecht zu werden, wird analog obigem zu $A \in \mathcal{K}$ und $\beta: A \rightarrow \mathcal{K}$ die Klasse $A \times \beta$ aller Paare $(a, b)$ mit $a \in A$ und $b \in a \beta$ eingeführt.

4.4. Jetzt ist klar, wie ,zusammen“ in der vorläufigen Definition des Klassenbegriffs zu eliminieren ist. Für den Erfolg entscheidend ist dabei, daß für Bereiche $A, B$ die Aussage $A=B(\Longleftrightarrow x \in A \Leftrightarrow x \in B)$ mit den Eigenschaften reflexiv, symmetrisch, transitiv zur Verfügung steht, daher auch für Relationen $\rho, \tau$ auf $A: \quad \rho=\tau \Longleftrightarrow x \rho=x \tau$ für alle $x \in A$.

Dedekinds Vorbild folgend (und Bolzanos in Fußnote 38 wiedergegebenem Prinzip) geben wir also den Begriff einer Klasse „als Individuo“ vorübergehend auf und führen stattdessen gleich die ganze "Gattung“ ein. Setze voraus:

(a) einen Bereich $\mathcal{K}$, dessen Elemente Klassen genannt werden,

(b) eine Abbildung, die jeder Klasse $K$ einen Bereich $K_{\mathcal{B}}$ zuordnet, den Grundbereich von $K$,

(c) eine Abbildung, die jeder Klasse $K$ eine Äquivalenzrelation $={ }_{K}$ auf $K_{\mathcal{B}}$ zuordnet, die Gleichheitsbeziehung von $K$,

(d) eine 2-Funktion, die jedem Bereich $A$ und jeder Äquivalenzrelation $\rho$ von $A$ eine Klasse $A / \rho$ mit $(A / \rho)_{\mathcal{B}}=A$ und $=_{A / \rho}=\rho$ zuordnet.

Wie früher kann nun $K=L\left(\Longleftrightarrow K_{\mathcal{B}}=L_{\mathcal{B}}\right.$ und $\left.={ }_{K}==_{L}\right)$ definiert werden, und eine explizit vorauszusetzende entsprechende Äquivalenzrelation $\rho$ würde $\mathcal{K}$ zu einer Klasse machen. 
4.5. Die Diskussion des Klassenbegriffes möchte ich mit Cantors Definition des ursprünglichen Mengenbegriffes abschließen [5]:

Unter einer „Menge“ verstehen wir jede Zusammenfassung $M$ von bestimmten wohlunterschiedenen Objekten $m$ unserer Anschauung oder unseres Denkens (welche die „Elemente“ von $M$ genannt werden) zu einem Ganzen.

Das lese ich so: Damit von einer Menge ${ }^{37} M$ geredet werden kann, müssen über zwei Definitionen die Elemente von $M$ zuerst bestimmt, dann unterschieden worden sein. Konkret ist also die Aussage $x \in M$ zu definieren, dann die Aussage $x=y$, und schließlich ist für $=$ noch reflexiv, symmetrisch, transitiv nachzuweisen (um „wohl“ zu respektieren).

Nichts trifft die mathematische Realität genauer. Das „Unterscheiden“ der Elemente geht allerdings im Alltag schnell vergessen, weil nach einigen Grunddefinitionen nur noch Teilmengen "gebildet" werden und diese ihre Gleichheitsbeziehung von der jeweiligen Obermenge erben.

4.6. Wie ist auf der Grundlage des Klassenbegriffs der Begriff einer Menge einzuführen? Soll eine Menge eine Klasse mit gewissen Eigenschaften sein? Weitere "Axiome" wären dann Voraussetzungen über $\mathcal{K}$ und als solche durchaus kein Eingriff in die mathematische Natur (Klasse ist nach 4.4 kein absoluter Begriff mehr). Natürlicher ist es aber doch, eine Klasse $\mathcal{M}$ von Klassen vorauszusetzen (eine Teilklasse von $\mathcal{K}$ ) - deren Elemente Mengen genannt werden mit den gewünschten Eigenschaften. Das entspricht durchaus dem Begriff eines Modells der axiomatischen Mengenlehre.

Was ist ein solches Modell? Ein Bereich, ein System, eine Gesamtheit von Dingen, die Mengen genannt werden? Werden solche Modelle gelegentlich, etwa zwecks Konstruktion weiterer Modelle, naiv-mengentheoretischen Prozessen unterworfen, deren Fundierung durch geeignete „Axiome“ doch eigentlich das Anliegen der axiomatischen Mengenlehre ist, jedenfalls Zermelos.

Wittenberg (S. 54): In den Arbeiten zur axiomatischen Mengenlehre äußert sich dies darin, dass dort «meta»-Betrachtungen angestellt werden, Überlegungen über die aufgestellten Axiomatiken, die inhaltlich unter Verwendung einer Terminologie geführt werden, die das Unbehagen des Verfassers deutlich durchschimmern läßt. Da ist dann etwa von «Gesamtheiten» die Rede, oder von «Systemen», als ob sich der mit Notwendigkeit an den inhaltlichen Mengenbegriff knüpfende Aufbau der Cantorschen Mengenlehre, mit seinen Problemen, einfach dadurch bannen ließe, dass man die Verwendung des Wortes «Menge» meidet. In Wirklichkeit ändert dies aber an der Sache überhaupt nichts. Menge bleibt Menge, auch wenn man sie «Gesamtheit» nennt.

\footnotetext{
${ }^{37}$ Lieber würde ich Klasse sagen.
} 
4.7. Mit irgendwas muß man halt anfangen, mit irgendwelchen undefinierten Grundbegriffen. Zu dieser An- und Einsicht äußert sich Wittenberg so:

S. 179: Einen Begriff reduzieren, heisst, ihn definitorisch aus anderen Begriffen erklären, also für ihn eine Definition konstruieren. Es ist wohl ohne weiteres klar, daß auf eine solche Weise keine generelle Lösung unseres Problems geleistet werden kann. Das Sinnproblem kann nicht durch logische Reduktionen aus der Welt geschafft werden. Nirgends liegt der Regress so klar zutage wie hier: Zur Definition eines Begriffes brauchen wir andere (einfachere), zu deren Definition wiederum noch primitivere usw., und wenn dieses ganze System von Definitionen nicht zirkulär sein darf - was wir selbstverständlich fordern werden -, so finden wir uns in einem unendlichen Regress, der nur dann zu vermeiden ist, wenn wir ihn irgendwo abbrechen, indem wir gewisse Begriffe als ultime Begriffe deklarieren - Begriffe, die undefiniert bleiben, und für die wir uns auf den Standpunkt stellen müssten, sie gehörten zum unerschütterlichen und unerklärbaren Rüstzeug unseres Verstandes. Wir müssten also annehmen, dass wir eine gewisse Anzahl von Begriffen gewissermassen als unsere persönliche Mitgift besitzen und alle übrigen aus diesen definitorisch in mehr oder weniger komplizierter Weise aufgebaut haben. Jene Begriffe stellten die letzte, unerschütterliche und undiskutierbare Grundlage unseres Denkens dar.

- - - Es kommt hinzu, dass hier noch ein zusätzliches Problem dadurch entstehen würde, dass man eine Auslese treffen müsste, welches denn nun eigentlich jene ultimen Begriffe seien. Dabei zeigt bereits das Beispiel der Mathematik, dass man sich auf erhebliche Schwierigkeiten gefasst zu machen hätte, weil einerseits verschiedenartige Möglichkeiten für die Annahme letzter Begriffe bestehen und andererseits gewisse Begriffe, die zu ultimen Begriffen prädestiniert scheinen (wie der mathematische Begriff der Menge oder jener der Eigenschaft), zu Schwierigkeiten und Antinomien führen. Nach welchen Kriterien sollte man hier entscheiden?

Es kann auch gar keine Rede davon sein, dass abzusehen wäre, wie eine solche generelle Reduktion durchzuführen wäre - auch angenommen, dass man einen Bestand an «Urbegriffen» ausgezeichnet hätte. Die vorstehende Konzeption bleibt auch insofern vollkommen hypthetisch und belanglos, als in keiner Weise ersichtlich ist, wie denn eine Zurückführung unseres ganzen begriffichen Materials auf einige Grundbegriffe tatsächlich durchgeführt werden könnte. (Ganz zu schweigen von der Notwendigkeit, bei einem solchen Standpunkt auch den Begriff der «Definition» einer Kritik zu unterwerfen.)

4.8. Ich setze dagegen: Bereich und Abbildung sind die kanonischen Urbegriffe der Mathematik, auf einer noch zu besprechenden logischen Grundlage.

Sie entstehen aus der realen mathematischen Begriffswelt durch maximales Vergessen, maximal weil auch der kleinste weitere Vergessensakt ins Nichts 
führt, aber auch bezüglich der Forderung, daß aus dem Nichtvergessenen immer noch diese Begriffswelt definitorisch rekonstriert werden kann. Kurz:

Mathematik ist die Theorie der Bereiche und Abbldungen.

Das Vergessene schließt jede inhaltliche Vorstellung ein. Jene Urbegriffe sind also rein sprachlicher Natur. Sie bleiben nicht undefiniert. Nicht was ein Bereich, ein Element, eine Abbildung is t, ist mathematisch relevant, sondern nur, wie mit Aussagen wie $A$ ist ein Bereich, $x$ ist ein Element von $A, \alpha$ ist eine Abbildung umzugehen ist. ${ }^{38}$ Dementsprechende Voraussetzungen stellen durchaus eine Definition dar, und zwar in folgendem Sinne:

(**) Eine Definition ist eine zwischenmenschliche Vereinbarung (letzlich eine Voraussetzung) über den logischen - genauer implikatorischen - Umgang mit einem (oder mehreren) Zeichen, einem Wort oder ähnlichem.

Ein kleiner Test (bevor der Leser die Nase rümpft):

Im Lichte von $(* *)$ werfe man einen (Rück)Blick auf das, was man gemeinhin „Definieren" von Mengen und Abbildungen nennt.

Erinnerung: Eine Definition rechtfertigt sich wie ein Licht, das in einem dunklen Raum aufgeht, durch die Ordnung, die sie sichtbar macht.

In der im Raume stehenden Synthese kommt es nur noch auf das Definieren von Aussagen an, und nach den ersten Schöpfungstagen werden Definitionen nur noch notationelle Bedeutung haben, d.h. auf sprachliche Abkürzungen hinauslaufen.

4.9. Was ich unter einer Menge (im engeren Sinne, im Sinne jener Synthese) verstehe, kann ich noch nicht ganz, aber doch schon weitgehend formulieren:

Setze eine Teilklasse $\mathcal{M}$ von $\mathcal{K}$ voraus (ihre Elemente heißen Mengen). In den „Axiomen“ (a) - (g) sind $A$ und $B$ immer Mengen, und $K$ ist eine Klasse.

(a) Die Aussage $x=y$ ist definit für $x, y \in A$.

(b) Teilklassen und Faktorklassen von $A$ sind Mengen (Teil/Faktormengen).

(c) Die Klasse $\mathcal{P}(A)$ aller Teilmengen von $A$ ist eine Menge.

(d) $\mathcal{F}(A, B)$ und $\mathcal{F}(A, \sigma)$ sind Mengen für $\sigma: A \rightarrow \mathcal{M}$.

(e) $A \times B$ und $A \times \sigma$ ebenso.

(f) $\alpha: A \rightarrow K$ surjektiv impliziert $K \in \mathcal{M}$.

(g) $\alpha: K \rightarrow A$ injektiv impliziert $K \in \mathcal{M}$.

\footnotetext{
${ }^{38}$ Bolzano: Ich denke also, daß man die Mathematik am besten als eine Wissenschaft erklären könnte, die von den allgemeinen Gesetzen (Formen) handelt, nach welchen sich die Dinge in ihrem Dasein richten müssen.

..., so zeiget dies an, daß unsre Wissenschaft sich nicht mit dem Beweise des Daseins dieser Dinge, sondern nur ganz allein mit den Bedingungen ihrer Möglichkeit beschäftige. Und indem ich diese Gesetze allgemeine nenne, so gebe ich zu verstehen, daß sich die Mathematik niemals mit einem einzelnen Dinge als Individuo, sondern allezeit mit ganzen Gattungen befasse.
} 
Erläuterung (jetzt mit Klassen $A, B$ und $\sigma: A \rightarrow \mathcal{K}$ ):

$\mathrm{Zu}$ (a): definit wird gleich in 5.3 erklärt.

$\mathrm{Zu}$ (b): Faktorklassen wurden de facto schon in 3.6 eingeführt

$\mathrm{Zu}(\mathrm{c})$ : Die Funktion $\mathcal{P}$ ordnet jeder Klasse die Klasse aller Teilklassen zu.

$\mathrm{Zu}(\mathrm{d})$ : Die Klasse $\mathcal{F}(A, B)$ aller Abbildungen von $A$ in $B$ wird über eine 2-Funktion (etwa $\mathcal{F}$ ) eingeführt. Die Gleichheitsbeziehung ist natürlich

$$
\alpha=\beta \Longleftrightarrow x \alpha=x \beta \text { für alle } x \in A \text {. }
$$

Dasselbe gilt für das kartesische Produkt $\mathcal{F}(A, \sigma)$ der Klassen $x \sigma(x \in A)$, bestehend aus den zu $A$ und $\sigma$ gehörigen Auswahlfunktionen [4.2].

$\mathrm{Zu}(\mathrm{f})$ : Surjektiv setzt eigentlich Existenz voraus. Wenn aber Objekt und Element nur Worte sind (ohne Inhalt), welchen Sinn hat es dann, von der Existenz solcher „Dinge“ zu reden? Genauso viel wie a priori von ihrer Gleichheit, nämlich überhaupt keinen. Man könnte surjektiv hier so verstehen:

Für jedes $u \in K$ ist die Aussage $\quad x \alpha \neq u$ für alle $x \in A$ falsch.

Inwieweit entspräche das der allgemeinen Existenzdefinition in 5.4(b)?

Was „Teilklasse von $\mathcal{K}$ " konkret bedeutet, hängt nicht von der Klasse $\mathcal{K}$ ab. Letzten Endes müssen wir auf sie verzichten, auch auf die Bereiche $\mathcal{B}$ und $\mathcal{D}$.

\section{Existenz - Negation - Eigenschaften.}

5.1. Es ist interessant, mit welchen Metaphern Existenz (Dasein, DA sein) in verschiedenen Sprachen umschrieben wird: Im Deutschen heißt es ES gibt, im Englischen THERE is, und das lateinische existere bedeutet entstehen, hervortreten. Wörtlich genommen, beschreiben sie exakt, wie Existenz in der Mathematik funktioniert: Wenn ES etwa eine Funktion $f$ mit gewissen Eigenschaften gibt (oder uns freundlicherweise schon gegeben hat), oder wenn DA ein solches $f$ ist, sozusagen auf dem Tisch liegt, für uns entstanden, hervorgetreten ist, dann bedienen wir uns dieses $f$ nach Herzenslust und erachten selbstverständich alles mit seiner Hilfe bewiesene als wahr.

Im Laufe der Zeit haben sich diese Metaphern als Existenzbegriff in unser Denken eingefressen. Sprachliche Gewohnheiten kreieren Begriffe.

Nietzsche: Ein Begriff ist eine gefrorene Metapher.

5.2. Unsere Aufgabe ist zum Glück aber keine sprachphilosophische.

Sie lautet Analyse-Synthese. Sehen wir uns also an, wie Existenz in der r. e. M. rein logisch gesehen funktioniert. Die Aussage

(a) Es gibt ein Objekt $x$ mit der Eigenschaft $E$ impliziert jede Aussage $S$, welche aus der Aussage

(b) $x$ ist ein Objekt mit der Eigenschaft $E$ folgt:

(c) (b) $\Rightarrow S \Longrightarrow S$. 
Ein Beispiel für $S$ ist (b) selbst, und jedenfalls folgt (a) aus (b).

Erhalte $(\mathrm{a}) \Rightarrow(\mathrm{c}) \Rightarrow(\mathrm{b}) \Rightarrow$ (a).

Die Äquivalenz (a) $\Leftrightarrow$ (c) scheint sich als Definition für die Existenzaussage (a) nahezulegen, aber die grausliche Konsequenz (a) $\Rightarrow(b)^{39}$ läßt Zweifel aufkommen. Kann man ihr entrinnen, etwa indem „Aussagen“ vom Typ (b) der Status einer (mathematischen) Aussage verweigert wird? Für mich eine hanebüchene Vorstellung: Nur Aussagen können mittels impliziert, und, oder $(\Rightarrow, \wedge, \vee)$ zu Aussagen verknüpft werden.

Für $S \Rightarrow T$ und $S \wedge T$ braucht $T$ dabei nur als Folge der Aussage $S$ eine Aussage zu sein, wie ein Blick auf die Realität zeigt:

Wenn $G$ eine Gruppe ist, und $U$ eine Untergruppe von $G$, und $N$ ein Normalteiler von $G$, dann ist $U \cap N$ ein Normalteiler von $U$.

Hier ist $G$ Gruppe $+U$ Untergruppe (von $G$ ) $+N$ Normalteiler (von $G$ ) eine Aussage, und ihr zufolge auch $U \cap N$ Normalteiler von $U$.

Wir halten fest, im Vorgriff auf die kommende ausführlichere Diskussion der Frage Was ist eine Aussage?:

(d) Ist $S$ eine Aussage und infolge $S$ auch $T$, so sind auch $S \Rightarrow T$ und $S \wedge T$ Aussagen (natürlich mitsamt allen Synonymen).

Bei $S \vee T$ muß neben $S$ auch $T$ von vornherein eine Aussage sein.

Aussagen wie (b) - etwa $G$ ist eine Gruppe oder $p$ ist eine Primzahl - sind uns nur als Quellaussagen geläufig, d.h. als Voraussetzung, als Beschreibung einer zu betrachtenden Situation (es sei denn, $G$ wurde etwa als Halbgruppe vorgegeben oder $p$ als natürliche Zahl). Unser Gefühl sträubt sich gegen das Attribut falsch im Zusammenhang mit solchen Aussagen, aber nur aus Gewohnheit, weil wir gewöhnlich eben (a) statt (b) negieren. Tatsächlich wird aber die Negation von (b) bewiesen, durch Herleitung eines Widerspruchs aus (b):

(e) (a) ist falsch $\Longleftrightarrow$ (b) impliziert eine falsche Aussage.

Wie steht es mit dem „tertium non datur"? $G$ ist entweder eine Gruppe oder nicht, $p$ ist entweder eine Primzahl oder nicht; sowas, mit nichts davor, ist schon als Aussage schwer zu schlucken, geschweige denn als wahre Aussage.

Wie man sich auch zu den angesprochenen Fragen stellt - man mag von logischen Spitzfindigkeiten reden - es bleibt ein Unbehagen über die mangelnde innere Harmonie unserer Logik. Wie stelle ich mich dazu, in diesem Augenblick? Nun, in (d) habe ich wohlweislich nur von und, oder, impliziert gesprochen, nicht von nicht.

\footnotetext{
${ }^{39}$ Lustigerweise ist das ganz in Ordnung wenn es gibt wieder aufgetaut wird: ES ist der Geber.
} 
5.3. Kommt ein Begriff ins Gerede, ist er ein Kandidat für das früher (in 2.3) beschriebene Verfahren, unter dem Aspekt Analyse-Synthese auf den Prüfstand gestellt und schließlich über eine geeignete Charakterisierung eliminiert zu werden, und nichts ist leichter als für die Negation $S^{f}$ ( $S$ ist falsch) einer Aussage $S$ eine einfache Charakterisierung anzugeben:

(a) $S^{f} \Longleftrightarrow S \Rightarrow F$,

wobei $F$ irgendeine falsche Aussage ist. Um (a) zu einer Definition zu erheben, ${ }^{40}$ muß ein geeignet erscheinendes $F$ ausgewählt werden. Dafür bietet sich die Aussage an, daß alle Aussagen äquivalent sind: $S, T$ Aussagen $\Longrightarrow S \Rightarrow T$.

Erstaunlicherweise sind die ableitbaren logischen Regeln aber fast ganz unabhängig von $F$. Nach der Festlegung ist $F$ sozusagen das Urfalsche, dem Urmeter in Paris vergleichbar. Ein weiterer, mir eigentlich sympathischerer Kandidat für $F$ ist die Aussage, daß alle Bereiche gleich sind. Da die relevanten Aussagen grundsätzlich bereichstheoretischer Natur sind, ${ }^{41}$ macht es praktisch keinen Unterschied.

Weitere Definitionen [(b) und (c)] und Regeln:

(b) $S$ ist definit $: \Longleftrightarrow S \vee S^{f}$,

(c) $S$ ist stabil $: \Longleftrightarrow S^{f f} \Rightarrow S$.

(d) (1) $S$ ist nicht instabil: $\left(S^{f f} \Rightarrow S\right)^{f f}$.

(2) $S$ ist nicht indefinit: $\left(S \vee S^{f}\right)^{f f}$.

(3) $S$ stabil stabil $\Longrightarrow S$ stabil.

(4) $S$ definit stabil $\Longrightarrow S$ definit.

(e) Das Assoziativgesetz für Stabilität:

$$
(S \Rightarrow T) \text { stabil } \Longleftrightarrow S \Rightarrow(T \text { stabil }) .
$$

(f) Die Lügnerregel: $\left(S \Leftrightarrow S^{f}\right)^{f}$.

Trivial und fundamental: Was etwas falsches impliziert, ist selber falsch.

Ich habe hier ein paar Regeln mit einem gewissen Unterhaltungswert ausgewählt. Für das gleich weitergeführte Thema Existenz ist zunächst nur

(g) $S^{f f f} \Leftrightarrow S^{f}$

von Interesse, d.h. jede Negation ist stabil $\left(S \Rightarrow S^{f f}\right.$ gilt immer).

Daß die stabilen Aussagen die quasi anständigen sind, geht schon daraus hervor, daß nur sie sich einem Widerspruchsbeweis herkömmlicher Art beugen:

Wenn die Negation $S^{f}$ eines behaupteten Satzes $S$ zu einem "Widerspruch" führt, zu einer falschen Aussage, so folgt zunächst nur $S^{f f}$.

\footnotetext{
${ }^{40}$ Hilbert: Eine Aussage heißt falsch, wenn sie auf einen Widerspruch führt [13].

${ }^{41}$ Mathematik ist die Theorie der Bereiche und Abbildungen [4.8].
} 
5.4. Jetzt kann jede Aussage ohne das geringste Unbehagen negiert, das begriffskritische Thema Existenz daher leicht seinem kanonischen Abschluß zugeführt werden. Die Nichtexistenz wird durch 2(e) charakterisiert:

(a) $2(\mathrm{a})^{f} \Leftrightarrow 2(\mathrm{~b})^{f}$.

Wie die Regeln $S^{f f f} \Leftrightarrow S^{f}[3(\mathrm{~g})]$ und $S \Rightarrow S^{f f}$ zeigen, ist auch $2(\mathrm{~b})^{f f}$ eine, und zwar die schwächste, Aussage $S$ mit $S^{f} \Leftrightarrow 2(a)^{f}$.

Damit bietet sich 2(b) $)^{f f}$ als Definition für die Existenzaussage 2(a) an:

(b) $2(\mathrm{a}): \Leftrightarrow 2(\mathrm{~b})^{f f}$.

Das Hauptbeispiel $x \in A$ ( $A$ ein Bereich) für $E$ entscheidet die Sache:

Nichtexistenz bedeutet $(x \in A)^{f}$, und das entspricht genau der Leere von $A$ (die Vorgabe $x \in A$ führt zu einem Widerspruch, zu $F$ ). Die Nichtleere von $A$ bedeutet also $(x \in A)^{f f}$, und das ist genau die Existenz von $x \in A$ (die eines Objektes $x$ mit der Eigenschaft $x \in A$ ) in dem eben angedachten Sinne.

Die mystische und inakzeptable Äquivalenz $2(\mathrm{a}) \Leftrightarrow 2(\mathrm{~b})$ - sie beruht auf $2(\mathrm{a}) \Leftrightarrow 2(\mathrm{c})$ - löst sich nun dadurch auf, daß 2(c) nur noch für stabiles $S$ gilt. So modifiziert bleibt 2(c) sogar äquivalent zu 2(a).

Existenzaussagen $2(\mathrm{~b})^{f f}$ sind stabil (jede Negation ist stabil), und nach $3(\mathrm{e})$ bleibt die Stabilität erhalten, wenn ihnen noch (wie eigentlich immer) eine Voraussetzung vorausgeht. Was $E$ angeht, so reicht

(c) $x \in \mathcal{D} \Rightarrow E$ ist eine Aussage

für unsere Diskussion völlig aus. Und es folgt sogar noch

(d) $\left(\exists_{x \in \mathcal{D}} E\right)^{f} \Leftrightarrow \forall_{x \in \mathcal{D}} E^{f}$,

(e) $\left(\forall_{x \in \mathcal{D}} E\right)^{f} \Leftrightarrow \exists_{x \in \mathcal{D}} E^{f}$,

wobei $\forall_{x \in \mathcal{D}} E$ für $x \in \mathcal{D} \Rightarrow E$ steht, $\exists_{x \in \mathcal{D}} E$ für die Existenz eines Objektes $x$ mit der "Eigenschaft" $E$, im Sinne von (b), d. h. im Sinne von $(x \in \mathcal{D} \wedge E)^{f f}$.

Natürlich dürfte $\mathcal{D}$ auch irgendein Bereich sein, oder sonst irgendeine Einschränkung der betrachteten Objekte $x$ andeuten.

Der Beweis von (d)(e) ist eine schlichte Anwendung der logischen Regeln

$$
(S \wedge T)^{f} \Longleftrightarrow S \Rightarrow T^{f} \quad(S \Rightarrow T)^{f} \Longleftrightarrow\left(S \wedge T^{f}\right)^{f f}
$$

neben $3(\mathrm{~g}){ }^{42}$ Dabei muß $T$ nicht wie $S$ als Aussage vorausgesetzt werden, es genügt $S \Longrightarrow T$ ist eine Aussage [2(d)]. Damit (c) als Aussage akzeptiert werden kann, muß genau wie etwa $A$ ist ein Bereich, $\alpha$ ist eine Abbildung, $x$ ist ein Objekt, $x \in A$ ( $A$ ein Bereich) auch $E$ ist eine Aussage eine Aussage sein. Salopp ausgedrückt: In Aussagen darf auch von Aussagen geredet werden, genau wie von Bereichen, Abbildungen, Objekten. Das war schon nötig, als wir von dem Urfalschen $F$ sprachen (alle Aussagen sind äquivalent).

\footnotetext{
${ }^{42}$ Das bestätigt die Kanonizität meiner Haltung zu Negation und Existenz.
} 
5.5. Die strikte Befolgung des in 2.6 formulierten Existenzprinzips Die Existenz eines mathematischen Objekts muß vorausgesetzt oder bewiesen werden, tertium non datur!

ist neben der Abkehr von einem inhaltlichen Mengenbegriff ${ }^{43}$ das herausragende Merkmal von Zermelos "Axiomatisierung“ der Mengenlehre [20]. Sie ist die zwangsläufige Folge dieser Abkehr. Zentral ist sein

Axiom der Aussonderung: Ist die Klassenaussage $\mathcal{E}(x)$ definit für alle Elemente einer Menge $M$, so besitzt $M$ immer eine Untermenge $M_{\mathcal{E}}$, welche alle diejenigen Elemente $x$ von $M$, für welche $\mathcal{E}(x)$ wahr ist, und nur solche als Elemente enthält.

$\mathrm{Zu}$ dem verwendeteten Begriff definit heißt es vorher:

Eine Frage oder Aussage $\mathcal{E}$, über deren Gültigkeit oder Ungültigkeit die Grundbeziehungen des Bereiches ${ }^{44}$ vermöge der Axiome und der allgemeingültigen logischen Gesetze ohne Willkür entscheiden, heißt „definit". Ebenso wird auch eine "Klassenaussage" $\mathcal{E}(x)$, in welcher der variable Term $x$ alle Individuen einer Klasse $K$ durchlaufen kann, als „definit" bezeichnet, wenn sie für jedes e inz elne Individuum der Klasse $K$ definit ist. So ist die Frage, ob $a \in b$ oder nicht ist, immer definit, ebenso die Frage, ob $M \subseteq N$ oder nicht.

Welcher Teufel ritt Zermelo, als er seine „Klassenaussage“45 mit dem Zusatz definit verzierte, der ihm statt Anerkennung nur Ärger und Mühe einbrachte [21]. Ich kann seinen Sinn ebensowenig erkennen wie seine Nachfolger, ${ }^{46}$ die aber die Notwendigkeit erkannten, den Begriff „Klassenaussage“ im Sinne einer für die jeweiligen Elemente definierten Eigenschaft zu erklären, d. h. zu definieren (oder das Axiom sonstwie umzuformulieren). Über das Ergebnis kann der Leser sich via [3] informieren. Siehe auch [9] (Zermelos Kritik [Fußnote 15] richtet sich offenbar gegen Fraenkels Funktionsbegriff).

Zermelos fruchtlose Versuche, sein „definit" zu erklären, schreien geradezu nach einer expliziten Definition für die Negation einer Aussage, genau wie die von Wittenberg [2.4] angesprochenen Diskussionen und Streitereien um das tertium non datur (plus Existenz), und genau so wie die Probleme mit dem Konvergenzbegriff nach einer expliziten Definition desselben drängten. Das sozusagen als Nachhall zu 5.3 und 5.4.

${ }^{43}$ Das gleich auftauchende Wort „Bereich“ ist diesbezüglich belanglos, auf [21] und [22] trifft Wittenbergs Vorwurf [4.6] aber durchaus zu.

${ }^{44}$ Zermelo: Die Mengenlehre hat zu tun mit einem „Bereich" von Objekten, die wir einfach als "Dinge" bezeichnen wollen, unter denen die "Mengen" einen Teil bilden.

${ }^{45}$ Verstehe darunter einen „den Term” $x$ involvierenden Ausdruck, der für $x \in K$ eine Aussage darstellt. Das entspricht dem praemodernen Funktionsbegriff der Analysis.

${ }^{46}$ Allerdings glaubte ich über 20 Jahre hinweg fest an die Zweckmäßigkeit der Einschränkung definit bei dem gleich folgenden Aussonderungsaxiom für Bereiche, sah in dieser Kombination geradezu den Eckstein der kommenden Synthese (den Stein der Weisen, Wort in Fleisch verwandelnd). 
5.6. Die Wortwahl Klassenaussage paßt gut zu unserem Klassenbegriff. Wenn ich den Begriff einer Eigenschaft oder Klassenaussage auf einer Klasse (speziell einer Menge) $A$ definieren müßte, dann als eine Abbildung $E$, die jedem $x \in A$ eine Aussage $E(x)$ zuordnet und natürlich eine Klassenabbildung sein soll: $x=y \Longrightarrow E(x) \Leftrightarrow E(y)$.

Dafür müßten Aussagen auch Objekte sein; am besten führt man gleich die Klasse aller Aussagen ein, mit $\Leftrightarrow$ als Gleichheitsbeziehung.

Ich muß aber nicht, weil es nämlich die Alternative gibt, auf den Allgemeinbegriff Eigenschaft ganz zu verzichten und sich allein auf 5.4(c) zu gründen, bezogen auf einen Bereich $A$ also auf

(a) $x \in A \Longrightarrow E$ ist eine Aussage.

Mit $x$ ist auch $y$ eine Zahl. Das ist der Kern des altertümlichen (aber eleganten) Begriffs einer Funktion $y$ von $x$. Worin liegt der Unterschied zu (a)?

Drücke (a) auch so aus: $E$ ist eine Eigenschaft von $x \in A$.

Oder so: $E$ ist eine Aussage über $x \in A$.

Das Aussonderungsaxiom für Bereiche lautet dann so:

(b) Gilt (a), so existiert ein Teilbereich $U$ von $A$ mit

$$
x \in A \Longrightarrow x \in U \Leftrightarrow E .
$$

Damit hat sich de facto auch ein Aussonderungsaxiom für Klassen etabliert:

Wende (b) auf den Grundbereich $A$ einer Klasse $K$ an, verifiziere die Verträglichkeit von $U$ mit $=_{K}$, erhalte die Teilklasse $U /=_{K}$.

Jede Aussage ist ein Beispiel für $E$ in (a), und umgekehrt könnte $E$ auch als Aussage vorausgesetzt werden: ersetze $E$ durch $x \in A \wedge E$. Das Beispiel $F$ anstelle $E$ führt über (b) zur Existenz eines leeren Bereiches und einer leeren Klasse (gesetzt, es gibt überhaupt einen Bereich bzw. eine Klasse).

Damit hat sich das Thema Eigenschaften als begriffskritisches Problem für mich erledigt. Gewiß, mit einem derartig schwachen Ersatz für einen „richtigen“ Eigenschaftsbegriff läßt sich nicht viel Staat machen, dafür klebt $x$ zu fest an $E$. Er reicht aber für einige allgemeine Begriffsbildungen, Voraussetzungen und Regeln, und in der Anwendung auf ein konkretes $E$ (man denke wie Zermelo an einen „den Term“ $x$ involvierenden Ausdruck, eine Art „Funktion von $x^{\text {") }}$ wird $x$ dann auch richtig ,variabel".

Übrigens: Ergibt die Analyse einer „Mengendefinition" $U=\{x \in A \mid \ldots\}$ nicht als erstes, daß ... eine Aussage ist wenn , $x \in A$ " gegeben ist?

Und ist nicht ein Bereich genau das, was der Leser sich intuitiv unter einer „richtigen“ Eigenschaft (von Objekten) vorstellt? Man sage doch einfach „Objekt mit der Eigenschaft $U$ ” statt „Element des Bereiches $U$ ”.

Der (b) innewohnenende Witz besteht gerade darin, eine schwache Eigenschaft $E$ im Sinne von (a) in eine starke Eigenschaft in dem eben erklärten Sinne zu verwandeln, sie quasi hochzutransformieren. 
5.7. Existenz im Sinne von 4(b), d.h. im Sinne von

(a) $\quad \exists_{x \in \mathcal{D}} E \Longleftrightarrow(x \in \mathcal{D} \wedge E)^{f f}$,

gestattet leider nicht mehr die in 5.1 geschilderten Gebräuche, sondern wird praktisch nur über 2(c) wirksam, und nur für stabiles $S$ :

$$
\exists_{x \in \mathcal{D}} E \text { plus } x \in \mathcal{D} \wedge E \Rightarrow S(S \text { stabil }) \text { impliziert } S \text {. }
$$

Nur um eine stabile Behauptung zu beweisen, dürfen wir uns also wie gewohnt verhalten, ein existentes Objekt (die Aussage $x \in \mathcal{D} \wedge E$ ) einfach voraussetzen, speziell $x \in A$ wenn $A$ ein nichtleerer Bereich ist.

Auch in der realen Welt, der Wirklichkeit, bedeutet das reine Sein nichts, nur die Wirkung zählt. Aus ihr wird überhaupt erst auf das Sein geschlossen. Letzten Endes wird es nur postuliert, als Ursache des Wahrgenommenen (des für wahr genommenen), der Sinnesempfindung, empfunden als Reaktion des Realen. Kurz: Im Anfang war das Wort, und das Wort ward Fleisch.

Noch kürzer: Cogitor, ergo sum.

Hugo v. Hofmannsthal: Daß wir Deutschen das uns Umgebende als ein Wirkendes - die «Wirklichkeit» bezeichnen, die lateinischen Europäer als «Dinglichkeit», la réalité, zeigt die fundamentale Verschiedenheit des Geistes, und daß jene und wir in ganz verschiedener Weise auf dieser Welt zu Hause sind. Siehe auch $\S 4$ in Schopenhauers Die Welt als Wille und Vorstellung I.

\section{Was ist eine Aussage?}

6.1. Aus seinen Betrachtungen zieht Wittenberg das Resümee, daß in der Mathematik weder der inhaltliche noch der rein formale Standpunkt voll zu befriedigen vermag. Er sieht diesen Konflikt auf der Mengenebene. Ich sehe ihn tiefer, in dem, was ich Reine Logik nenne. Darunter verstehe ich die Art und Weise, wie Menschen über Aussagen an sich reden, also ohne Rücksicht auf den eventuellen Inhalt der jeweiligen Aussagen, und zwar allein mit den Worten und, oder, wenn ... dann (und ihren Synonymen). Sie gehört also zu der jeder mathematischen Kommunikation zugrundeliegenden Sprachkultur.

Alles Eliminieren (Vergessen plus Synthese) findet dort sein natürliches Ende, wo kein weiterer derartiger Schritt ohne das eigentlich Vergessene zu bewerkstelligen ist. Dadurch bestimmen sich die Grenzen der „Reinen Logik“. Man mag von Aussagenlogik reden, aber ohne Negation, sie reduziert sich also auf die Angabe der banalsten logischen Regeln. Aber: Die Aussagenlogik ist nur der komprimiert geschriebene Ausdruck des realen Denkens. ${ }^{47}$

Es ist also ganz gleichgültig, ob man diese Regeln angibt - ins Bewußtsein hebt - oder nicht: Ihre korrekte Anwendung, ihr richtiges Verständnis, setzt das voraus, was durch sie vermittelt werden soll. Schon die Aufführung von

\footnotetext{
${ }^{47}$ Warum kann Hänschen nicht rechnen? M. Kline, Beltz-Verlag, Basel 1974.
} 
Regeln (1), (2), .. über Aussagen $A, B, C, \ldots$ ist doch so zu verstehen: WENN $A, B, C, \ldots$ Aussagen sind, DANN GILT (1) UND (2) UND ....

Neben dem „richtigen“ Gebrauch und Verständnis der involvierten Worte (wenn ...dann, es gilt, und) involviert diese allerniedrigste Stufe auch schon geläufige Symbole - hier A, B, C-als Variable oder Platzhalter, als "Namen“ oder „Bezeichnungen“ für alles mögliche.

Läßt sich auch nichts weiter reduzieren, so kann es doch durchaus nützlich sein, sich noch einmal vor Augen zu halten, daß das Wörtchen und einem schon vorhandenen Wissen weiteres (eine weitere Information) hinzufügt, und daß die Wahrheit einer Implikation $S \Rightarrow T$ darauf hinausläuft, daß ein gedachtes Gegenüber mit $S$ auch $T$ als „wahr" zur Kenntnis nimmt, mein „also gilt $T$ " zustimmend abnickt, und zwar ohne oder mit meiner Hilfe ${ }^{48}$ (Beweis genannt), je nachdem ob $S \Rightarrow T$ schon bekannt ist (als wahr akzepiert wurde) oder nicht.

Mathematische Wahrheit läuft also auf ein gewisses durch Gewöhnung entstandenes Zustimmungsverhalten hinaus. ${ }^{49}$ Mehr kann auch ein „externer Beobachter der mathematischen Szene" [2.6] nicht gewahr werden.

Schon wenn ich etwas eine Regel nenne, erwarte ich, daß der Leser sie als wahr anerkennt, so wie die Regel $S \Leftrightarrow S$ ist wahr, die die Unsinnigkeit jeglicher Wahrheitsdefinition besonders deutlich macht.

Die der Mathematik nachgesagte Präzision, ihr so gut wie absoluter Wahrheitsgehalt, ihre Widerspruchsfreiheit, läuft einfach auf die Homogenität dieses Zustimmungsverhaltens hinaus, auf eine durch Begriffsarmut ermöglichte Sprachdisziplin. Kommt es doch einmal zu einem Widerspruch, zu einer Zustimmungsverweigerung, so genügt es, den Widersprechenden (den Zweifler, den Schweiger) zu ignorieren, als inkompetent oder verrückt (von der geraden, richtigen Linie abweichend, abrückend, weggerückt) zu erklären. ${ }^{50}$ Man muß ihn zum Schutze der Wahrheit nicht gleich einsperren oder verbrennen.

Trotz einer weiteren Äußerung Klines im Nacken, ${ }^{51}$ möchte ich doch noch kurz den Umgang mit „Variablen“ ansprechen:

\footnotetext{
${ }^{48}$ Galileo Galilei: Man kann einen Menschen nichts lehren, man kann ihm nur helfen, es in sich selbst zu entdecken.

${ }^{49}$ Dieses geht letztlich auf die schon im Tierreich erworbenenen Erfahrungen mit der zeitlichen Abfolge von Ereignissen zurück. Die logische Bedeutung der Worte folgen und wenn ... dann abstrahiert ihren urspünglich rein temporalen Sinn.

Siehe auch Kapitel X (Sprache und Wirklichkeit in der modernen Physik) in dem Büchlein Physik und Philosophie von W. Heisenberg [Ullstein Verlag, Berlin 1961].

${ }^{50}$ Oder als einen als Mathematiker verkleideten Philosophen [12].

${ }^{51}$ Die Neue Mathematik als Ganzes ist eine Darstellung aus der Sicht des seichten Mathematikers, der lediglich die unbedeutenden deduktiven Details schätzt, sowie die minderen pedantischen, sterilen Unterscheidungen, wie diejenige zwischen Zahl und Zahldarstellung, und der danach trachtet, Bagatellen durch hochtönende Terminologie und Symbolismus aufzubauschen.
} 
Dem Ausdruck $\forall_{x \in A} E$ - für jedes Element $x$ von $A$ gilt $E$ - habe ich den Inhalt $x \in A \Rightarrow E$ gegeben. In der Praxis gibt es einen kleinen Unterschied: Angesichts $\forall_{x \in A} E$ brauche ich mich nicht um eine früher dem Symbol $x$ gegebene Bedeutung zu kümmern. Ein auf den ersten Blick gruppentheoretischer Satz $G$ Gruppe $\Rightarrow \ldots$ hat eine ganz andere Bedeutung, wenn in dem gegebenen Zusammenhang schon $G$ als Halbgruppe vorliegt.

6.2. Wir haben im Laufe der Analyse alles auf Elementaraussagen wie $A$ ist ein Bereich, $x$ ist ein Element von A, $x$ ist ein Objekt, $\alpha$ ist eine Abbildung, $S$ ist eine Aussage reduziert. Von letzterer abgesehen, ist ihr Informationsgehalt rein formaler Natur, d.h. die Worte Bereich, Element, Objekt, Abbildung würden Dedekinds Probe [2.1, Fußnote 11] mühelos bestehen. Dagegen ist das Konglomerat Aussage, und, oder, wenn/dann inhaltlicher Natur. Es verankert die Mathematik im engeren Sinne in der zugrundeliegenden Sprachkultur.

Was immer uns in Fortsetzung des bisherigen als Aussage begegnen wird, läßt sich als sukzessive aus solchen Elementaraussagen via 5.2(d) - Komposition mittels und, oder, impliziert - entstanden denken. ${ }^{52}$ Eine solche Beschreibung in eine Definition umzumünzen, habe ich schon in 2.4 ausgeschlossen. Wie im Kefersteinbrief würde Falschgeld entstehen, Papier mit den inhaltsleeren Worten „karam sipo tatura“. Auch als bare Münze genommen würde es nichts an dem Begriffsproblem ändern, sondern nur die mathematischen aus der Masse der gewöhnlichen - umgangssprachlich so genannten - Aussagen aussondern, mittels expliziter Angabe statt implizit durch Angabe einiger Regeln.

Dieser Gegensatz ist der zwischen dem Äußerlichen und dem Innerlichen in der Mathematik [Ges. Werke II, 54-55], personifiziert durch Hilbert und Dedekind. Dessen Aversion gegen das Äußerliche (im Einklang mit einem Gottesgebot: Du sollst dir kein Bildnis machen!) steht Hilberts Betonung des konkret-anschaulichen an seiner Beweistheorie diametral gegenüber.

Der Aufgabe, den Begriff einer Aussage zu erklären, stehe ich letztlich genauso hilflos gegenüber wie der hl. Augustinus der Frage Was ist die Zeit?.

So wie es dem Physiker genügt, die Zeit zu messen, muß es dem Mathematiker genügen, jedesmal wenn er es von seinem Gegenüber erwartet, eine Äußerung, einen Ausdruck, als Aussage akzeptiert zu sehen. Und das sollten die oben angesprochenen Festlegungen (5.2(d) plus Elementaraussagen) mit einer für das mathematische Schließen ausreichenden Schärfe gewährleisten.

${ }^{52}$ Auf einem solchen Verständnis ließe sich dann eine Art Beweistheorie etablieren. Eine solche, auf welcher Grundlage auch immer, ist Meta-Mathematik in des Wortes natürlicher Bedeutung, oder, nach entsprechender Axiomatisierung, eine mathematische Theorie. Der Vergleich mit theoretischer bzw. mathematischer Physik ist vielleicht nicht ganz abwegig. Als abwegig empfinde ich nur die Vermengung mit dem Thema Grundlagen der Mathematik. 
Daß sich die Innereien einer Aussage so dem anatomisch interessierten Auge verschließen, daß man nicht einmal von ihnen reden kann, verbaut jede Möglichkeit, sich mit seinen Grundlagenproblemen in eine wie auch immer geartete logische Wissenschaft zu flüchten (wie in der axiomatischen Mengenlehre).

Man verfügt nur über das blanke Wort Aussage, nicht über ein-, zwei-, oder mehrstellige „Prädikate“ oder sonstwas.

6.3. Die in der Literatur verstreuten logisch-sprachlichen Antinomien beruhen hauptsächlich darauf, daß alles und jedes als Aussage akzeptiert wird, daß auch noch die Lebenssituation der aussagenden Personen mit ins Spiel kommt. Außerdem wird mit schwammigen Begriffen gearbeitet, und manchmal sind die Dinge auch gar nicht richtig durchdacht, rein logisch gesehen.

Witzig und lehrreich ist die folgende Version des Lügnerparadoxons ${ }^{53}$ :

Sokrates: Was Platon gleich sagen wird, ist falsch.

Platon: Sokrates hat wahr gesprochen.

Das Lehrreiche an der Sache besteht darin, daß sie die früher gegebene Definition $S^{f}: \Longleftrightarrow S \Rightarrow F$ mühelos überlebt. Anstelle eines Widerspruchs folgt nun die ganz beliebige Aussage $F$ (etwa der Satz von Fermat). Das Vergnügen der Herleitung lasse ich aber dem Leser. Die Aussage des Sokrates lautet jetzt

(S) Die gleich folgende Aussage des Platon impliziert $F$.

In seinem Buche Aufbau der Physik [Carl Hanser Verlag, München 1985] entwickelt C. F. v. Weizsäcker eine "Logik zeitlicher Aussagen“ und wendet sie auf ein "altbekanntes $\gg$ Paradox «" an: Der Lehrer sagt den Schülern: $\gg$ In der kommenden Woche werde ich eine Klassenarbeit schreiben lassen, aber ihr werdet nicht vorher wissen, an welchem Tag.« Präzisierungsfrage: $\gg$ Werden wir es auch am Morgen des betreffenden Tages nicht wissen? Antwort: $\gg$ Auch an dem Morgen nicht.« Das Paradox besteht nun darin, daß diese Aussage

1. einen Widerspruch impliziert,

2. empirisch leicht bestätigt werden kann.

Um es kurz zu machen: Die Schüler schließen, mit dem letzten beginnend, einen Tag nach dem andern aus, und wundern sich dann, als der Lehrer am Mittwoch doch schreiben läßt. So stellen sich jedenfalls die Autoren die „empirische Bestätigung" vor.

Aber: Eure Gedanken sind nicht unsere Gedanken!

Jedem aufgeweckten Schüler ist es Mittwoch früh sonnenklar, daß die Arbeit noch am selben Tage geschrieben werden muß, weil ja alle übrigen Tage logisch einwandfrei ausgeschlossen wurden.

${ }^{53}$ Dem Büchlein Die Scheinwelt des Paradoxons von P. Hughes und G. Brecht entnommen [Vieweg, Braunschweig 1978].

In ihm wird auch das gleich folgende ,altbekannte $\gg$ Paradox $\ll$ " diskutiert. 
6.4. Das unausgesprochen im Raume stehende Wort Unfug, einhergehend mit Dedekinds karam sipo tatura, erinnert an einen Passus in Zermelos [21]:

Natürlich gibt es auch „nicht-definite" Eigenschaften in jedem System, wie etwa „grün angestrichene“ Mengen oder Irrationalzahlen, „,ie durch keine endliche Anzahhl von Worten in einer beliebigen europäischen Sprache definiert werden können", und es erscheint mir keineswegs überflüssig, auf solche „unsinnigen“ oder „unwissenschaftlichen" Definitionen Bezug zu nehmen: hat man doch mit solchen Hilfsmitteln u. a. „die Unmöglichkeit der Wohlordnung des Kontinuums" nachweisen wollen.

Ich denke, man sollte jedem sein Vergnügen lassen, der sich mit der Menge der grünen Zahlen zwischen 5 und 99 beschäftigen möchte. Es lohnt sich auch nicht, die Existenz einer solchen Menge zu bestreiten; das wäre genauso unsinnig wie sie zu behaupten (aber nicht unsinniger als Existenz- und Gleichheitsfragen in manch anderem Bereich).

Ähnlich sehe ich die Menge aller Zahlen $y$ mit $x+2=5$. Zu ihr gelangt man ganz seriös so: Schreibe $E$ für $x+2=5$. Dann ist $E$ eine Aussage wenn (immer wenn) $x$ eine Zahl ist, und letztere Aussage ( $x$ Zahl $\Rightarrow E$ Aussage), für sich genommen, impliziert somit dasselbe mit $y$ anstelle $x$, d. h. $E$ ist auch eine Eigenschaft von Zahlen $y$ (in der Terminologie von 5.6). Ist das bedenklich?

6.5. Zurück zum Ernst des Lebens! Dedekind im Kefersteinbrief:

Damit war die Analyse beendigt, und der synthetische Aufbau konnte beginnen; es hat mir doch noch Mühe genug gemacht!

Auch der Leser meiner Schrift hat es wahrlich nicht leicht; außer dem gesunden Menschenverstande gehört auch noch ein sehr starker Wille dazu, um Alles vollständig durchzuarbeiten.

Also auf zum Aufbruch in das neue Land. ${ }^{54}$ Manch exotisch anmutendes mag dem kühnen Entdecker begegnen, und auch dafür hält das Buch von Weizsäcker und Juilfs [3.1] ein schönes Wort parat:

Wer sich die Welt anschaulich vorstellen will, begnüge sich wie Goethe mit dem wirklichen Augenschein, mit dem Urphänomen.

Wer hinter den Augenschein dringen will, suche dort nicht die Gesetze des Bereiches wiederzufinden, den zu verlassen sein Ziel ist.

Mutatis mutandis: Man nehme das Thema Grundlagen der Mathematik ernst, oder lebe weiter glücklich und zufrieden in Cantors Paradies.

\footnotetext{
${ }^{54}$ Der Teil und das Ganze. W. Heisenberg. R. Piper \& Co., München 1969.
} 



\section{Bereiche - KLASSen - Mengen}

\section{Reine Logik und Negation.}

1.1. Unsere Diskussion (mathematischer) Aussagen gründet sich auf die Annahmen (a) und (b) und den gewohnten Umgang mit den Worten und, oder, impliziert (und ihren Synonymen, wie $\wedge, \vee, \Rightarrow$ ), sowie mit den als Namen für alles mögliche vertrauten Symbolen, meistens Buchstaben.

Dieser „gewohnte Umgang“ läuft auf ein gewisses homogenes Zustimmungsverhalten hinaus, in welchem sich für mich auch der Begriff mathematischer Wahrheit erschöpft. Es liegt jeder zwischenmenschlichen Kommunikation zugrunde, also auch jedem Versuch es zu erklären, und darin ändert auch die Auflistung schöner logischer Regeln nichts.

Wir nutzen die vertraute mathematische Sprache, soweit ihre Bedeutung in dem hier entwickelten Rahmen klar ist.

Natürlich soll (b) auch für jedes andere „Symbol“ anstelle $X$ gelten, und das läßt sich auch so ausdrücken: ist eine Aussage ist ein Prädikat (im gewöhnlichen grammatischen Sinne) der zu entwickelnden Sprache. Weitere Prädikate werden in 2.1 eingeführt: ist ein Objekt, ist ein Bereich, ist eine Abbildung.

(a) Mit $S$ und $T$ sind auch $S \wedge T, S \vee T$ und $S \Rightarrow T$ Ausssagen. Für $\wedge$ und $\Rightarrow$ genügt anstelle $T$ Aussage die Voraussetzung

(*) $\quad S$ hat zur Folge, daß $T$ eine Aussage ist.

(b) $X$ ist eine Aussage ist eine Aussage.

1.2. Bezüglich einer noch festzulegenden Aussage $F$ wird die Negation $S^{f}$ einer Aussage $S$ als die Aussage $S \Rightarrow F$ definiert, und daraus erklärt sich die Bedeutung von Worten wie nicht und falsch im Zusammenhang mit Aussagen. Weitere Definitionen:

$S$ ist stabil $: \Longleftrightarrow S^{f f} \Rightarrow S$,

$S$ ist definit $: \Longleftrightarrow S \vee S^{f}$.

Das kanonische $F$, das Urfalsche, ist die Aussage, daß alle Aussagen äquivalent sind: $A, B$ Aussagen $\Longrightarrow A \Rightarrow B$. Es liegt allem weiteren zugrunde. Gleichwertig: Eine falsche Aussage impliziert jede Aussage.

Ohnehin ist falsch was etwas falsches impliziert, und auch die Masse der gleich folgenden Regeln ist ganz unabhängig von $F$ : setze in 1.4(b), 1.5(b) sowie $1.5(\mathrm{a})[\Rightarrow]$ noch $F \Rightarrow T$ voraus. All diese Regeln könnten einem auch bei den Intuitionisten begegnen, die übrigens das Wort stabil in ähnlichem Sinne verwendeten (laut Meyers Konversationslexikon).

Obiges $(*)$ genügt soweit dies überhaupt infrage kommt:

In 1.4(a)(b)(d $\left.\mathrm{d}^{\prime}\right), 1.5(\mathrm{a})\left(\mathrm{b}^{\prime}\right), 1.6(\mathrm{~d}), 1.8$, und in 1.6(a) für $\wedge$ und $\Rightarrow$. 
1.3. Einfache Grundregeln.
(a) $S \Rightarrow T \Longrightarrow T^{f} \Rightarrow S^{f}$.
(b) $S \Rightarrow T \Longrightarrow S^{f f} \Rightarrow T^{f f}$.
(c) $S \Rightarrow S^{f f} \quad\left(S \wedge S^{f}\right.$ ist falsch $)$.
(d) $S^{f} \Leftrightarrow S^{f f f} \quad\left(S^{f}\right.$ ist stabil $)$.
(e) $S \Rightarrow S^{f} \Longrightarrow S^{f}$.
(f) $\left(S \Leftrightarrow S^{f}\right)^{f} \quad$ (Lügnerregel).

1.4. Negation von $\wedge \vee \Rightarrow$.
(a) $(S \wedge T)^{f} \Longleftrightarrow S \Rightarrow T^{f}$.
(b) $(S \Rightarrow T)^{f} \Longleftrightarrow\left(S \wedge T^{f}\right)^{f f}$.
(c) $(S \vee T)^{f} \Longleftrightarrow S^{f} \wedge T^{f}$.
(d) $(S \wedge T)^{f} \Longleftrightarrow\left(S^{f} \vee T^{f}\right)^{f f}$.
$\left(\mathrm{d}^{\prime}\right)(S \wedge T)^{f} \Longleftrightarrow\left(S^{f} \vee\left(S \Rightarrow T^{f}\right)\right)^{f f}$.

1.5. Doppelte Negation.
(a) $(S \Rightarrow T)^{f f} \Longleftrightarrow S \Rightarrow T^{f f}$.
(b) $(S \wedge T)^{f f} \Longleftrightarrow S^{f f} \wedge T^{f f}$.
$\left(\mathrm{b}^{\prime}\right)(S \wedge T)^{f f} \Longleftrightarrow S^{f f} \wedge\left(S \Rightarrow T^{f f}\right)$.
(c) $(S \vee T)^{f f} \Longleftrightarrow S^{f} \Rightarrow T^{f f}$.

1.6. Stabil und definit.
(a) Mit $S$ und $T$ sind auch $S \wedge T, S \vee T, S \Rightarrow T$ definit.
(b) Ist $T$ definit, so ist $T$ stabil (also auch $T^{f}$ definit).
(c) Ist $S$ definit und $T$ stabil, so ist auch $S \vee T$ stabil.
(d) Mit $S$ und $T$ ist auch $S \wedge T$ stabil.

1.7. Mehr darüber.
(a) $\left(S \vee S^{f}\right)^{f f} \quad(S$ ist nicht indefinit $)$.
(b) $\left(S^{f f} \Rightarrow S\right)^{f f} \quad(S$ ist nicht instabil).
(c) $S$ ist definit $\Longleftrightarrow S$ ist definit ist stabil.
(d) $S$ ist stabil $\Longleftrightarrow S$ ist stabil ist stabil.

1.8. Stabilität ist assoziativ:

$$
(S \Rightarrow T) \text { ist stabil } \Longleftrightarrow \quad S \Rightarrow(T \text { ist stabil }) .
$$


1.9. Ein stabiles $T$ folgt schon aus $S^{f f}$ wenn es aus $S$ folgt [1.3(b)]. Damit ist $S^{f f}$ (stabil und von $S$ impliziert) der stabile Abschlu $\beta$ von $S$.

1.10. Ein Standardbeweis ist ein Beweis einer stabilen Aussage. In einem solchen darf $S$ vorausgesetzt werden wenn $S^{f f}$ gilt.

Ein Beispiel tut es für alle: Das erste Assoziativgesetz 1.5(a) führt nämlich via $1.7(\mathrm{a})(\mathrm{b}) \mathrm{zu}$

$$
(S \text { Aussage } \Rightarrow S \text { ist stabil und definit })^{f f}
$$

und somit darf (und wird) in einem Standardbeweis jede Aussage als stabil und sogar definit angenommen werden. Mit $S^{f f}$ ist somit auch $S$ gegeben.

Das zweite Assoziativgesetz 1.8 folgt sofort aus dem ersten:

Die linke Seite $L$ bedeutet $S \Rightarrow T \Longleftrightarrow(S \Rightarrow T)^{f f}$ und damit $S \Rightarrow T \Longleftrightarrow$ $S \Rightarrow T^{f f}$. Und die rechte Seite $R$ bedeutet $S \Rightarrow\left(T \Leftrightarrow T^{f f}\right)$.

Ich verifiziere noch die etwas anspruchsvolleren Regeln 1.4(b)(d) und gebe zum Rest ein paar Hinweise.

$\mathrm{Zu} \mathrm{1.4(b):} \mathrm{Zum} \mathrm{Beweis} \mathrm{von} \Longrightarrow$ nehmen wir (die linke Seite) $L$ an, dann $R^{\prime}: \Leftrightarrow\left(S \wedge T^{f}\right)^{f}$ zum Beweis von $R$, mit dem Ziel $F$.

Laut $L$ genügt $S \Rightarrow T$. Nehme also $S$ an, jetzt mit Ziel $T$. Dafür genügt $F$, denn $F$ impliziert alles. Laut $R^{\prime}$ genügt $S \wedge T^{f}$, also schon $T^{f}$.

Nehme also $T$ an, mit Ziel $F$. Aus $T$ folgt $S \Rightarrow T$, und daraus $F$ via $L$.

Für die Rückrichtung $\Longleftarrow$ wird $R$ angenommen plus $S \Rightarrow T$. Zum Beweis von $F$ via $R$ wird obiges $R^{\prime}$ nachgewiesen. Dazu wird $S \wedge T^{f}\left[S\right.$ und $\left.T^{f}\right]$ angenommen, wieder mit Ziel $F$. Mit $S$ gilt $T$, und $T$ plus $T^{f}$ impliziert $F$.

Übrigens gilt $R \Leftrightarrow\left(S \Rightarrow T^{f f}\right)^{f}$, aufgrund (a).

Zu 1.4(d): Für $\Longrightarrow$ wird neben $L$ noch $R^{\prime}: \Leftrightarrow\left(S^{f} \vee T\right)^{f}$ angenommen, mit dem Ziel $F$. Ist $S$ gegeben, so folgt $T^{f}$ (aus $L$ ), und aus $T^{f}$ folgt $F$ via $R^{\prime}$.

Das beweist $S^{f}$, also $F$, wieder wegen $R^{\prime}$.

Es bleibt $R \Rightarrow L$. Nehme also $R$ und $S \wedge T$ an, mit Ziel $F$.

Dafür genügt $R^{\prime}$. Nehme also $S^{f} \vee T^{f}$ an, wieder mit Ziel $F$, und dieses ergibt sich sofort aus $S \wedge T$.

$\mathrm{Zu}\left(\mathrm{d}^{\prime}\right)$ : Wende (d) mit $S \wedge T$ anstelle $T$ an und beachte (a).

1.5(a): Wende 1.4(b)(a) an und $X^{f f f} \Leftrightarrow X^{f}[1.3(\mathrm{~d})]$.

1.5(b): Wende 1.4(d)(c) an und wieder $X^{f f f} \Leftrightarrow X^{f}$.

$\mathrm{Zu}\left(\mathrm{b}^{\prime}\right)$ : Wende (b) an mit $S \Rightarrow T$ anstelle $T$ und beachte (a).

1.5(c) folgt sofort aus 1.4(c)(a), 1.6(c) aus 1.5(c), 1.6(d) aus 1.5(b), 1.7(a) aus 1.5(c), 1.7(b) aus 1.5(a), 1.7(c) aus (a), und 1.7(d) aus (b).

Es bleibt 1.6(a)(b). Man muß bei $\wedge$ und $\Rightarrow$ wie andernorts (Liste in 1.2) nur darauf achten, daß $(*)$ ausreicht. Die Varianten $\left(\mathrm{d}^{\prime}\right)$ und $\left(\mathrm{b}^{\prime}\right)$ in 1.4 und 1.5 sind natürlich nur deshalb aufgeführt, weil für sie $(*)$ ausreicht. 


\section{Bereiche und Abbildungen.}

2.1. Wie in 1.1 angedeutet, ist $x$ ist ein Objekt eine Aussage, ebenso $A$ ist ein Bereich und $\alpha$ ist eine Abbildung (wobei $\alpha$ bzw. A ein Objekt ist).

Der rein sprachliche Informationsgehalt wird durch (a)(b) wiedergegeben:

(a) $x \alpha$ ist ein Objekt wenn $x$ ein Objekt und $\alpha$ eine Abbildung ist,

(b) $x \in A$ ist eine Aussage wenn $x$ ein Objekt und $A$ ein Bereich ist.

Das reflektiert das absolute Minimum an Information, welches mit den Begriffen Menge und Abbildung in der r.e. Mathematik verbunden ist:

Eine Menge $M$ bzw. Abbildung $\alpha$ erlaubt, von den Elementen von $M$ zu reden bzw. den Bildern $x \alpha$ gewisser Objekte $x$.

Das Wort Funktion - synonym mit „Abbildung“ - verwende ich gerne für individuelle, dem Funktionieren des Ganzen dienende Abbildungen.

2.2. Für Bereiche $A, B$ haben $A \subseteq B$ und $A=B$ die vertraute Bedeutung:

$$
x \in A \Rightarrow x \in B \quad \text { bzw. } \quad x \in A \Leftrightarrow x \in B,
$$

und $\alpha: A \rightarrow B$ steht für $x \in A \Rightarrow x \alpha \in B$.

Die Beziehungen $=$ und $\subseteq$ (reine Notation!) sind reflexiv und transitiv, weiter symmetrisch bzw. anti-symmetrisch.

(a) $\alpha: A \rightarrow B$ plus $A^{\prime} \subseteq A$ impliziert $\alpha: A^{\prime} \rightarrow B$.

(b) $\alpha: A \rightarrow B$ plus $B \subseteq B^{\prime}$ impliziert $\alpha: A \rightarrow B^{\prime}$.

2.3. Eine Relation $z u$ einem Bereich $A$ ist eine Abbildung $\rho$ der Art, daß $x \rho$ für jedes $x \in A$ ein Bereich ist; und $\rho$ ist eine Relation auf oder von $A$, wenn $x \rho$ sogar ein Teilbereich von $A$ ist $(x \in A \Rightarrow x \rho \subseteq A)$.

Schreibe $x \rho y$ für $y \in x \rho$. Das erklärt den Begriff einer Äquivalenzrelation.

Auch für Relationen werden die „Beziehungen" = und $\subseteq$ erklärt:

$$
\rho \subseteq(=) \tau \text { bedeutet } x \rho \subseteq(=) x \tau \text { für alle } x \in A,
$$

und das in 2.2 über $=$ und $\subseteq$ gesagte bleibt gültig.

(*) Sei $U \subseteq A$ und $\rho$ eine Relation von $A$ mit $x \rho \subseteq U$ für alle $x \in U$. Dann ist $\rho$ auch eine Relation von $U$.

2.4. Ein Bereich $A$ ist leer, wenn $(x \in A)^{f}$ gilt.

Dann folgt $A \subseteq B$ für jeden Bereich $B$, denn $x \in A$ ist falsch, impliziert also alles, insbesondere $x \in B$.

Läßt sich ein Element in $A$,wählen“ wenn $A$ nicht leer ist?

Ist eine aus $x \in A$ resultierende Aussage $T$ tatsächlich wahr?

Ja, wenn $T$ stabil ist, denn dann genügt $(x \in A)^{f f}$ statt $x \in A[1.9]$. 


\section{Existenz.}

3.1. Schreibe kurz $x \in \mathcal{D}$ für $x$ ist ein Objekt. Setze für das folgende

$(*) \quad x \in \mathcal{D} \Longrightarrow E$ ist eine Aussage

voraus. Das genügt für einige allgemeine Definitionen, Regeln und Voraussetzungen. Auf etwas explizites angewandt - man denke an einen $x$ involvierenden Ausdruck anstelle $E$ - lassen diese sich in gewohnter Weise handhaben, das bei allgemeinem $E$ fest daran klebende $x$ wird wieder "variabel“ ${ }^{55}$

Es liegt nahe, bei $E$ von einer Aussage über $x \in \mathcal{D}$ (über Objekte $x$ ) zu reden, auch von einer Eigenschaft von $x \in \mathcal{D}$. Dem Wort „Eigenschaft" an sich gebe ich aber keine formale Bedeutung. ${ }^{56}$

Natürlich könnte $\mathcal{D}$ auch für irgendeinen Bereich stehen, oder für sonst eine Einschränkung, eine weitere Aussage $E^{\prime}$ über Objekte $x$. Dazu wäre aber $(*)$ mit $E^{\prime} \wedge E$ anstelle $E$ äquivalent.

Es ist kein Verlust an Allgemeinheit, gleich eine Aussage $E$ vorauszusetzen: ersetze einfach $E$ durch $x \in \mathcal{D} \wedge E[1.1(\mathrm{a})(*)]$.

3.2. Schreibe $\exists_{x \in \mathcal{D}} E$ für $(x \in \mathcal{D} \wedge E)^{f f}$.

Die Negation dieser Existenzaussage ist $\quad(x \in \mathcal{D} \wedge E)^{f}[1.3(\mathrm{~d})]$.

Das Hauptbeispiel für $E$ ist $x \in A$ ( $A$ ein Bereich). Nichtexistenz bedeutet $A$ ist leer, Existenz somit $A$ ist nicht leer. Genauso vertraut ist uns

$$
x \in \mathcal{D} \wedge E \quad \Longrightarrow \quad \exists_{x \in \mathcal{D}} E
$$

und daß zum Beweis der Nichtexistenz eines Objektes $x$ mit Eigenschaft $E$ ein solches $x$ zu einem Widerspruch (einer falschen Aussage) geführt werden muß.

3.3. Schreibe $\forall_{x \in \mathcal{D}} E$ für $x \in \mathcal{D} \Rightarrow E$. Mit 1.4(a)(b) folgt

(a) $\left(\exists_{x \in \mathcal{D}} E\right)^{f} \Longleftrightarrow \forall_{x \in \mathcal{D}} E^{f}$,

(b) $\left(\forall_{x \in \mathcal{D}} E\right)^{f} \Longleftrightarrow \exists_{x \in \mathcal{D}} E^{f}$.

3.4. Der Beobachtung in 2.4 ganz analog ist die folgende (allgemeinere): $\exists_{x \in \mathcal{D}} E$ plus $x \in \mathcal{D} \wedge E \Rightarrow T(T$ stabil $)$ impliziert $T$.

3.5. Die Existenz eines Objektes $x$ mit Eigenschaft $E$ gelangt praktisch nur über 3.4 zur Anwendung: In einem Standardbeweis darf ein solches $x$ explizit vorausgesetzt werden.

Noch besser [1.10]: Mit $\exists_{x \in \mathcal{D}} E$ ist auch $x \in \mathcal{D} \wedge E$ gegeben.

Jede Existenzaussage $\exists_{x \in \mathcal{D}} E$ ist stabil, wie allgemeiner jede Negation $S^{f}$.

Gewöhnlich geht $\exists_{x \in \mathcal{D}} E$ noch eine Voraussetzung $V$ voraus, und auch eine solche allgemeine Existenzaussage $V \Rightarrow \exists_{x \in \mathcal{D}} E$ ist stabil [1.8].

\footnotetext{
${ }^{55}$ Wer aus $(*)$ die Aussage $y \in \mathcal{D} \Rightarrow E$ ist eine Aussage ableitet, mag sehen wie weit er damit kommt.

${ }^{56}$ Wem es nach einem „richtigen“ Eigenschaftsbegriff verlangt, möge doch einfach Objekt mit Eigenschaft $A$ sagen statt Element des Bereiches $A$.
} 


\section{Klassen und Gleichheit.}

4.1. In erster Näherung ist eine Klasse ein Bereich zusammen mit einer Äquivalenzrelation. Das Problem ist das Wort zusammen, dahinter der Begriff eines Paares. Wir setzen voraus:

(a) einen Bereich $\mathcal{K}$, dessen Elemente Klassen genannt werden,

(b) eine Funktion, die jeder Klasse $K$ einen Bereich „zuordnet“, den Grundbereich $K_{\mathcal{B}}$ von $K$,

(c) eine Funktion, die jeder Klasse $K$ eine Äquivalenzrelation von $K_{\mathcal{B}}$ zuordnet, die Gleichheitsbeziehung $=_{K}$ von $K$,

(d) eine Funktion (etwa/), die jedem Bereich $A$ eine Abbildung (etwa $A /$ ) zuordnet, die jeder Äquivalenzrelation $\rho$ von $A$ eine Klasse $A / \rho$ zuordnet mit

$$
(A / \rho)_{\mathcal{B}}=A \quad \text { und } \quad={ }_{A / \rho}=\rho .
$$

4.2. (a) Elemente $x, y$ einer Klasse $K$ heißen gleich wenn $x={ }_{K} y$ ist.

Schreibe einfach $x=y$, wie $x \in K$ statt $x \in K_{\mathcal{B}}$.

(b) Sei $U$ ein Teilbereich von $K_{\mathcal{B}}$ mit $x={ }_{K} \subseteq U$ für alle $x \in U$.

Dann ist $=_{K}$ auch eine Äquivalenzrelation von $U[2.3(*)]$, und daraus ergibt sich die Klasse $U /=_{K}$. Beispiele: $U=x={ }_{K}$ und leere Teilbereiche $U$.

Bezeichne die Klasse $x={ }_{K} /=_{K}$ mit $\{x\}_{K}$ oder kurz $\{x\}$.

(c) Eine Klasse $L$ ist eine Teilklasse von $K$ - schreibe $L \subseteq K$ - wenn

$$
L_{\mathcal{B}} \subseteq K_{\mathcal{B}} \text { und }\{x\}_{L}=\{x\}_{K} \text { für alle } x \in L .
$$

Dual hierzu ist $L$ eine Faktorklasse von $K$, wenn

$$
L_{\mathcal{B}}=K_{\mathcal{B}} \text { und }\{x\}_{L} \supseteq\{x\}_{K} \text { für alle } x \in L .
$$

Natürlich heißen $K$ und $L$ gleich - schreibe $L=K$ - wenn

$$
L_{\mathcal{B}}=K_{\mathcal{B}} \text { und }\{x\}_{L}=\{x\}_{K} \text { für alle } x \in L \text { (d.h. } x \in K \text {. }
$$

(d) Für Teilklassen $X, Y$ von $K$ gilt $X \subseteq Y \Leftrightarrow X_{\mathcal{B}} \subseteq Y_{\mathcal{B}}$.

(e) Um die Klasse $\mathcal{P}(K)$ aller Teilklassen von $K$ zu „definieren“, simultan für alle Klassen $K$, nehmen wir eine Funktion $\mathcal{P}: \mathcal{K} \rightarrow \mathcal{K}$ an mit

$$
X \in \mathcal{P}(K) \Longleftrightarrow X \subseteq K \quad \text { und } \quad X=_{\mathcal{P}(K)} Y \Longleftrightarrow X=Y .
$$

Die Teilklasse $\{K\}$ von $\mathcal{P}(K)$ besteht aus allen Klassen $L=K$.

(f) $\mathcal{M} \in \mathcal{K}$ ist eine Klasse von Klassen, auch Quasiteilklasse von $\mathcal{K}$ genannt, wenn jedes $X \in \mathcal{M}$ eine Klasse ist mit $\{X\}=\{X\}_{\mathcal{M}}$.

Beispiel: $\mathcal{M}=\mathcal{P}(K)$.

4.3. 4.1(d) erklärt den Schönfinkelkniff [17], d. h. wie eine ganz gewöhnliche Abbildung zwei, drei, oder mehr Dingen $a, b, c, \ldots$ etwas zuordnen kann, so als stünden Paare $(a, b)$, Tripel $(a, b, c), \ldots$ zur Verfügung.

Die 2-variable Funktion / in 4.1(d) ordnet $A$ und $\rho$ ein Element von $\mathcal{K}$ zu. 
4.4. (a) Für Klassen $K, L$ bedeutet $\alpha: K \rightarrow L$ neben $\alpha: K_{\mathcal{B}} \rightarrow L_{\mathcal{B}}$ noch $x \alpha=y \alpha$ für alle $x, y \in K$ mit $x=y$.

(b) Die Klasse $\mathcal{F}(K, L)$ all dieser Abbildungen, mit Gleichheitsbeziehung $\alpha=\beta \Longleftrightarrow x \alpha=y \beta \quad$ für alle $x \in K$,

wird analog 4.2(e) eingeführt: Setze eine 2-variable Funktion $f$ voraus, die $K$ und $L$ die gewünschte Klasse zuordnet; schreibe dann $\mathcal{F}(K, L)$ statt $L K f$, außerdem $\mathcal{F}(K)$ statt $\mathcal{F}(K, K)$.

(c) Auch für $\mathcal{K}$ anstelle $L$ werden die Aussagen $\alpha: K \rightarrow L$ und $\alpha=\beta$ definiert, genau wie oben, mit $\mathcal{K}$ anstelle $L_{\mathcal{B}}(\mathcal{K}$ anstelle $K$ ist irrelevant).

4.5. Eine Auswahlfunktion zu $\lambda: K \rightarrow \mathcal{K}$ ist eine Abbildung $\alpha$ mit $x \alpha \in x \lambda$ für alle $x \in K$, plus $x \alpha=y \alpha$ für alle $x, y \in K$ mit $x=y$.

Die Klasse all dieser $\alpha: K \rightarrow \lambda$, das kartesische Produkt $\mathcal{F}(K, \lambda)$ der Klassen $x \lambda(x \in K)$ wird analog $\mathcal{F}(K, L)$ eingeführt.

4.6. Zu Klassen $A, B$ wird das Produkt $A \times B \in \mathcal{K}$ analog 4.1 eingeführt:

Setze $P \in \mathcal{K}$ voraus sowie Funktionen $\pi_{1}: P \rightarrow A$ und $\pi_{2}: P \rightarrow B$ mit

$$
p=q \quad \Longleftrightarrow p \pi_{1}=q \pi_{1} \text { und } p \pi_{2}=q \pi_{2},
$$

ferner eine 2-variable Funktion $\pi$, die $a \in A$ und $b \in B$ ein $p(=b a \pi)$ in $P$ zuordnet mit $p \pi_{1}=a$ und $p \pi_{2}=b$. Schreibe $A \times B$ für $P$, und $p_{i}$ für $p \pi_{i}$.

Die universelle Verfügbarkeit solcher Klassenprodukte erfordert noch vier 2-variable Funktionen, welche $A, B \in \mathcal{K}$ die Objekte $P, \pi_{1}, \pi_{2}, \pi$ zuordnen, und diese Funktionen sollten sich mit $\subseteq$ vertragen:

Für $A^{\prime} \subseteq A$ und $B^{\prime} \subseteq B$ ist $P^{\prime}=A^{\prime} \times B^{\prime}$ eine Teilklasse von $P=A \times B$, und $\pi_{1}, \pi_{2}, \pi$ setzen ihre $A^{\prime}$ - $B^{\prime}$-Analoga $\pi_{1}^{\prime}, \pi_{2}^{\prime}, \pi^{\prime}$ fort.

Natürlich steht $(a, b)$ für $\pi(a, b)=b a \pi$, weiter $A \times B \times C$ für $((A \times B) \times C)$ und $(a, b, c)$ für $((a, b), c)$. Für $p=(a, b, c)$ folgt $a=p \pi_{1} \pi_{1}, b=p \pi_{1} \pi_{2}$ und $c=p \pi_{2}$, wobei $\pi_{i}$ sowohl für $\pi_{i}(A, B)$ als auch für $\pi_{i}(A \times B, C)$ steht.

Wir schreiben auch einfach $p_{1}, p_{2}, p_{3}$.

Wir müssen auch von „Paaren“ $(a, b)$ reden können, bei denen $b$ irgendwie von $a$ abhängt. Für $A \in \mathcal{K}$ und $\beta: A \rightarrow \mathcal{K}$ wird daher die Klasse $P=A \times \beta$ aller Paare $(a, b)$ mit $a \in A$ und $b \in a \beta$ eingeführt. Verfahre analog obigem.

4.7. Es liegt nahe, unter einer Relation einer Klasse eine mit der Gleichheitsbeziehung verträgliche Relation des Grundbereichs zu verstehen. Die folgende Variante wird jedoch im Vordergrund stehen:

Eine Relation zu einer Klasse $A$ ist eine Abbildung $\rho: A \rightarrow \mathcal{K}$.

Im Falle $\rho: A \rightarrow \mathcal{P}(A)$ ist $\rho$ eine Relation auf oder von $A$.

Natürlich steht $x \rho y$ wieder für $y \in x \rho$. 


\section{Existenz von Teilklassen und Abbildungen.}

5.1. Mit den folgenden „Axiomen" begegnen uns die ersten wirklich inhaltlichen Voraussetzungen. Die bisherigen Annahmen dienten nur der Einführung gewisser Grundobjekte und -begriffe.

Axiom I [Existenz von Teilbereichen].

Sei $A$ ein Bereich und $E$ eine Aussage über $x \in A$.

Dann hat $A$ einen Teilbereich $U$ mit

$$
x \in A \Longrightarrow x \in U \Leftrightarrow E .
$$

Axiom II [Existenz von Auswahlfunktionen].

$\mathcal{F}(K, \lambda)$ ist nicht leer, wenn all die Klassen $x \lambda(x \in K)$ nichtleer sind.

Axiom III [Existenz von Relationen].

Zu jeder Teilklasse $U$ von $A \times B$ oder $A \times \beta$ existiert die Funktion, welche jedem $a \in A$ die Klasse aller $b \in B$ bzw. $b \in a \beta$ mit $(a, b) \in U$ zuordnet.

5.2. (a) Axiom I wird gewöhnlich auf den Grundbereich $A$ einer Klasse $K$ angewandt, und ist dann nur von Interesse wenn (jedes) $U$ mit $=_{K}$ verträglich ist und damit zu einer Teilklasse $U /=_{K}$ führt [4.2(b)].

Diese Verträglichkeit ist einem explizit gegebenem $E$ sofort anzusehen, und läßt sich bei allgemeinem $E$ einfach so definieren, daß jedes $U$ verträglich ist.

Nenne $E$ dann eine $K$-Aussage/Eigenschaft über/von $x \in K$.

(b) Das Beispiel $F$ anstelle $E$ führt zur Existenz der leeren Teilklasse $\emptyset$. Ich sage "der" und nicht „einer", weil alle leeren Klassen „gleich" sind.

(c) $K=\emptyset$ bzw. $K \neq \emptyset$ dient einfach als Abkürzung für $K$ ist (nicht) leer.

(d) Sonstiges Auftreten von $\emptyset$ ist implizit mit der Annahme verbunden, daß $\emptyset$ eine leere Klasse ist. Diese Konvention ist immer zu beachten, wenn ein lediglich existentes Objekt die Bühne betritt. Aus der Sicht eines später folgenden Standardbeweises sind derartige Annahmen redundant [3.4/5].

5.3. Der $B$-Fall von Axiom III führt zu der konstanten Funktion $a \rightarrow B$, und dann über Axiom II, das Auswahlaxiom nach Zermelo, zu $\mathcal{F}(A, B) \neq \emptyset$ wenn $B \neq \emptyset$ oder $A=\emptyset$ ist. Für $b \in B$ ist also $\mathcal{F}(A,\{b\}) \neq \emptyset$, d. h. es existiert die konstante Funktion $a \rightarrow b$.

5.4. Ich wiederhole den in 5.2(a) erarbeiteten Existenzsatz für Teilklassen:

Ist $K$ eine Klasse und $E$ eine $K$-Aussage über $x \in K$, so hat $K$ eine Teilklasse $U$ mit $x \in K \Longrightarrow x \in U \Leftrightarrow E$.

Schreibe $U=\{x \in K \mid E\}$.

Damit wird nur eine Aussage definiert, eine Eigenschaft der Teilklasse $U$ von $K$. Der Ausdruck $\{\ldots \mid \ldots\}$ erhält erst in $\S 7$ unter gewissen Zusatzvoraussetzungen eine eigenständige Bedeutung. 
5.5. Sei wieder $A \in \mathcal{K}, \beta: A \rightarrow \mathcal{K}$ und $P=A \times \beta$. Setze eine Aussage $E$ über $x \in A$ und $y \in x \beta$ voraus, d. h.

(*) $\quad x \in A$ und $y \in x \beta \Longrightarrow E$ ist eine Aussage.

Ein explizit gegebenes $E$ läßt sich leicht in eine Aussage $E^{\prime}$ über $p \in P$ transformieren. Wäre $x+y=3$ ein Beispiel für $E$, so wäre $E^{\prime} \Leftrightarrow p_{1}+p_{2}=3$.

Ich arbeite lieber mit der Aussage $E^{\prime}: \Longleftrightarrow x \in A \wedge y \in x \beta \wedge E$.

Jede Aussage ist eine Aussage über $p \in P$. Somit hat $P$ einen Teilbereich $U$ mit $p \in U \Leftrightarrow E^{\prime}$ und folglich

$(* *) \quad x \in A$ und $y \in x \beta \Longrightarrow(x, y) \in U \Leftrightarrow E$.

Jetzt können wir analog 5.2(a) von einer $A$ - $\beta$-Aussage/Eigenschaft über/von $x \in A$ und $y \in x \beta$ reden, wenn jedes $(* *)$ genügende $U$ mit $=_{P}$ verträglich ist und somit zu der Teilklasse $U /=_{P}$ von $P$ führt. Auf diese läßt sich dann Axiom III anwenden, und daraus ergeben sich gewisse Abbildungen:

5.6. Existenzsatz für Abbildungen.

$\mathrm{Zu} A, \beta, E$ wie eben existiert $\sigma: A \rightarrow \mathcal{K}$ mit $x \sigma=\{y \in x \beta \mid E\}$.

Existiert zu jedem $x \in A$ ein $y \in x \beta$ mit $E$, so existiert ein $\alpha: A \rightarrow \sigma$, d.h. ein $\alpha: A \rightarrow \beta$ welches jedem $x$ ein $y$ mit $E$ zuordnet:

$$
x \in A, x \alpha=y \in x \beta \Longrightarrow E .
$$

Beides (wie folgendes) gilt auch für $B \in \mathcal{K}$ anstelle $\beta$ (und $x \beta$ ).

5.6'. Eine meta-mathematische Betrachtung zu 5.6.

$\mathrm{Zu}$ jedem $x \in A$ sei ein Element $f(x) \in x \beta$ explizit angegeben, und für $x^{\prime}=x$ gelte $f\left(x^{\prime}\right)=f(x)$. Wende 5.6 an mit $E: \Longleftrightarrow y=f(x)$.

Erhalte $\alpha: A \rightarrow \beta$ mit $x \alpha=f(x)$.

Das vertraute naive „Zuordnen“ läßt sich also durchaus über 5.6 realisieren.

Beispiel: Das Produkt $\alpha: x \rightarrow x \alpha_{1} \alpha_{2}$ von $\alpha_{1}: A \rightarrow M$ und $\alpha_{2}: M \rightarrow B$.

5.7. Der Fall $A=K \times \gamma$ in 5.6.

Sei $E^{\prime}$ eine Aussage über $x_{1} \in K, x_{2} \in x_{1} \gamma$ und $y \in\left(x_{1}, x_{2}\right) \beta$, natürlich wieder mit $=$ veträglich. Arbeite mit der Aussage

$$
x_{1} \in K \wedge x_{2} \in x_{1} \gamma \wedge y \in\left(x_{1}, x_{2}\right) \beta \wedge E^{\prime}
$$

anstelle $E$. Daraus resultiert $\sigma$ mit

$$
\left(x_{1}, x_{2}\right) \sigma=\left\{y \in\left(x_{1}, x_{2}\right) \beta \mid E^{\prime}\right\}
$$

und $\alpha$ folgt analog.

5.7'. Das $(A=K \times \gamma)$-Analogon von 5.6'.

Sei jetzt zu $x_{1} \in K$ und $x_{2} \in x_{1} \gamma$ ein Element $f\left(x_{1}, x_{2}\right) \in\left(x_{1}, x_{2}\right) \beta$ explizit gegeben (die „Pseudofunktion" $f$ wieder mit $=$ verträglich).

Dann existiert $\alpha: A \rightarrow \beta$ mit $\left(x_{1}, x_{2}\right) \alpha=f\left(x_{1}, x_{2}\right)$.

Arbeite dazu mit $F(x)=f\left(x \pi_{1}, x \pi_{2}\right)$ anstelle $f(x)$. 


\subsection{Anwendungen von 5.6.}

(a) Seien $A, B$ Klassen und $\rho$ eine Relation „zwischen” $A_{\mathcal{B}}$ und $B_{\mathcal{B}}$, d. h. für jedes $x \in A$ ist $x \rho$ ein Teilbereich von $B$. Außerdem sei $\rho$ mit $={ }_{A}$ und $={ }_{B}$ verträglich, d. h. für $x=x^{\prime}$ in $A$ und $y=y^{\prime}$ in $B$ gilt $\quad x \rho y \Leftrightarrow x^{\prime} \rho y^{\prime}$.

Wende 5.6 an mit $E \Leftrightarrow x \rho y$. Für $\sigma: A \rightarrow \mathcal{P}(B)$ gilt $x \sigma=x \rho /={ }_{B}$.

(b) Ein Beispiel für $\rho$ (mit $A=B$ ) ist $\rho==_{A}$. Hier ist $x \sigma=\{x\}$, und $\alpha: A \rightarrow A$ ist „die“ identische Abbildung von $A: x \alpha=x$ für alle $x \in A$.

Sie hätte auch gut als Beispiel zu 5.6' getaugt.

(c) Die Abbildung $\gamma: B \rightarrow A$ sei surjektiv $[x \in A \Rightarrow \exists y \in B$ mit $y \gamma=x]$.

Die $B$-Version von 5.6, angewandt mit $E: \Leftrightarrow y \gamma=x$, führt jetzt zu einem Linksinversen $\alpha: A \rightarrow B$ von $\gamma$ (für $x \in A$ ist $x \alpha \gamma=x$ ).

(d) Ein Fortsetzungssatz: Setze Klassen $A, B$ voraus sowie Teilklassen $U, U^{\prime}$ von $A$ mit $A=U \dot{\cup} U^{\prime}$. Zu $\lambda: U \rightarrow B$ und $\lambda^{\prime}: U^{\prime} \rightarrow B$ existert dann eine (und nur eine) Fortsetzung $\alpha: A \rightarrow B$ von $\lambda$ und $\lambda^{\prime}$.

Wende dazu 5.6 an mit $E: \Longleftrightarrow(x \in U \wedge x \lambda=y) \vee\left(x \in U^{\prime} \wedge x \lambda^{\prime}=y\right)$.

5.9. (a) Zwar steht in einem Standardbeweis die identische Abbildung $\operatorname{id}_{A}$ einer gegebenen Klasse $A$ zur Verfügung, auch die einer weiteren Klasse $A_{1}$, oder noch einer weiteren Klasse $A_{2}$, oder ..., aber die generelle Verfügbarkeit, sozusagen für alle Klassen gleichzeitig, setzt (cum grano salis) eine Funktion voraus, etwa id, die jedem $A$ sein $\operatorname{id}_{A}$ zuordnet.

Analog bräuchte man für die universelle Verfügbarkeit der Abbildung $a \rightarrow b$ in 5.3 eine 3 -variable Funktion, welche $A, B, b$ diese Abbildung zuordnet.

"Cum grano salis": Es könnte sich auch um eine Kombination von Funktionen handeln, wie bald in $\S 7$.

(b) Ein Existenzbeweis via 5.6 würde die Klasse aller Klassen voraussetzen, zumindest ihre Existenz, d. h. die Existenz einer Äquivalenzrelation $\rho$ des Bereiches $\mathcal{K}$ mit $A \rho B \Leftrightarrow A=B$.

Würde die Russellsche Antinomie die (Existenz der) Klasse $\mathcal{K}$ - genauer $\mathcal{K} / \rho$ - zulassen? Jedes Kind weiß heutzutage wie sie funktioniert: Die Klasse $\mathcal{R}$ aller Klassen $K$ mit $K \notin K$ führt zu einem Widerspruch.

Wohl existiert der Teilb er e ich $\mathcal{R}$ von $\mathcal{K}$, aufgrund Axiom I; die Existenz der Teilklasse $\mathcal{R}$ erfordert aber die Verträglichkeit der Eigenschaft $K \notin K$ mit $\rho$ [5.4], d. h. aus $A=B$ und $A \notin A$ müßte auch $B \notin B$ folgen, was ich nicht sehe (vgl. mit II.3.2). Nanu, wäre die Klasse $\mathcal{K}$ vielleicht doch lebensfähig?

(c) Leider nicht: Mit ihr stünde auch die Klasse $P=\mathcal{P}(\mathcal{K})$ zur Verfügung. Die Eigenschaft $\mathcal{L} \notin \mathcal{L}$ von $\mathcal{L} \in P$ ist mit $=_{P}$ verträglich. Nach 5.4 existiert daher die aus allen $\mathcal{L}$ mit $\mathcal{L} \notin \mathcal{L}$ bestehende Teilklasse $\mathcal{R}$ von $P$. Sie führt über die Lügnerregel 1.3(f), mit $S: \Leftrightarrow \mathcal{R} \in \mathcal{R}$, zu einem Widerspruch. 
(d) Das erledigt nicht nur $\mathcal{K}$ als Klasse, sondern auch noch die hypothetische Klasse $\mathcal{P}(\mathcal{K})$ aller Quasiteilklassen von $\mathcal{K}[4.2(\mathrm{f})]$.

(e) Ein weiteres Opfer Russells ist die hypothetische Klasse $F=\mathcal{F}(A, \mathcal{K})$ aller $\alpha: A \rightarrow \mathcal{K}$, wobei $A$ irgendeine nichtleere Klasse ist $\left[=_{F}\right.$ gemäß 4.4(c)].

Betrachte, um sie zu einem Widerspruch zu führen, zu $x \in A$ die nach 5.4 existente Teilklasse $R$ aller $\alpha$ mit $\alpha \notin x \alpha \subseteq F$ (in der Tat ist diese Eigenschaft von $\alpha \in F$ mit $=_{F}$ verträglich). Nach 5.3 existiert $\alpha$ mit $x \alpha=R$. Wende jetzt die Lügnerregel an mit $S: \Leftrightarrow \alpha \in R$.

(f) Der hypothetische Bereich $\mathcal{D}$ aller Dinge, d.h. Objekte, würde über Axiom I zu dem Bereich $\mathcal{B}$ aller Bereiche führen, und dieser zu dem Bereich $\mathcal{R}$ aller Bereiche $A$ mit $A \notin A$, dieser schließlich über die Lügnerregel, wiederum angewandt mit $S: \Leftrightarrow \mathcal{R} \in \mathcal{R}$, zu einem Widerspruch.

Die Option, $\mathcal{D}$ oder $\mathcal{B}$ anzunehmen, ist damit ausgeschlossen. Anders sähe es allerdings aus, würden wir uns mit 5.4 anstelle Axiom I begnügen.

(g) Es ist unfair, ein einzelnes Objekt oder einen Begriff an sich als in sich widersprüchlich zu diffamieren. Es ist immer ein ganzes Konglomerat von Begriffen und Voraussetzungen, von menschlichen Denk- und Verhaltensweisen, das zu einem Widerspruch führt.

Ist meine Verhaltensweise, was den Umgang mit Existenz angeht, korrekt? Wie wäre sie allein aufgrund 3.4 (ohne 1.10) zu beurteilen?

5.10. (a) Eine Faktorklasse $K / \rho$, genauer $K_{\mathcal{B}} / \rho$, einer Klasse $K$ erfordert eine Äquivalenzrelation $\rho$ des Grundbereiches $K_{\mathcal{B}}$ von $K$. Ohne eine passende Voraussetzung führt kein Weg von einer Klassenrelation $\rho$ zu der entsprechenden Bereichsrelation $\rho^{\prime}$ (mit $x \rho^{\prime} y \Leftrightarrow x \rho y$, d. h. $\left.x \rho^{\prime}=(x \rho)_{\mathcal{B}}\right)$.

(b) Deshalb setze ich eine 2-variable Funktion voraus, welche $K \in \mathcal{K}$ und $\rho: K \rightarrow \mathcal{K}$ die Relation $\rho_{\mathcal{B}}$ zu $K_{\mathcal{B}}$ zuordnet mit $x \rho_{\mathcal{B}}=x \rho_{\mathcal{B}}$ für alle $x \in K$.

Schreibe $K / \rho$ statt $K_{\mathcal{B}} / \rho_{\mathcal{B}}$, wenn $\rho$ eine Äquivalenzrelation von $K$ ist.

(c) Sei $\rho$ wie eben und $\tau$ irgendeine mit $\rho$ verträgliche Relation von $K$. Nach 5.6 existiert die Relation $\sigma$ (schreibe $\tau / \rho$ ) von $K / \rho$ mit $x \sigma=x \tau / \rho$, d. h.

$$
x \sigma y \Leftrightarrow x \tau y \text { für alle } x, y \in K .
$$

(d) Die identische Abbildung $\chi$ von $K$ ist zugleich surjektiv von $K$ auf $K / \rho$, und nach 5.8(c) existiert ein Linksinverses $\chi^{\prime}: K / \rho \rightarrow K$.

(e) Der geschmeidige Begriff einer Faktorklasse - und damit die ihn erst aktivierende Voraussetzung (b) - ist nicht wirklich wesentlich:

Die zu $\rho: K \rightarrow \mathcal{P}(K)$ gehörige Partition $K \rho$ von $K$ kann $K / \rho$ ersetzen.

$K / \rho$ als Ersatz für $K \rho$ anzupreisen ist mir allerdings Herzenssache.

Wesentlich ist alleine die Existenz einer Klasse $K^{\prime}$ mit einer $x^{\prime}=y^{\prime} \Leftrightarrow x \rho y$ genügenden Surjektion $x \rightarrow x^{\prime}$.

Erläuterung. $K \rho$ ist das noch zu „definierende“ Bild von $K$ unter $\rho$. 


\section{Mengen.}

Nehme eine Klasse $\mathcal{M} \neq \emptyset$ von Klassen an, Mengen genannt, weiter:

(1) Die Aussage $x=y$ ist definit für Elemente $x, y$ einer Menge.

(2) Teilklassen von Mengen sind Mengen (Teilmengen genannt).

(3) Faktorklassen von Mengen sind Mengen (Faktormengen genannt).

(4) Die Klasse $\mathcal{P}(A)$ aller Teilmengen einer Menge $A$ ist eine Menge.

(5) $A \times B$ und $A \times \beta$ sind Mengen für $A, B \in \mathcal{M}$ und $\beta: A \rightarrow \mathcal{M}$.

(6) Ebenso $\mathcal{F}(A, B)$ und $\mathcal{F}(A, \beta)$.

(7) $\alpha: A \rightarrow K$ surjektiv mit $A \in \mathcal{M}$ und $K \in \mathcal{K}$ impliziert $K \in \mathcal{M}$.

(8) $\alpha: K \rightarrow A$ injektiv mit $A \in \mathcal{M}$ und $K \in \mathcal{K}$ impliziert $K \in \mathcal{M}$.

Bezeichne diese "Mengenaxiome" mit $\mathcal{M}_{1}, \mathcal{M}_{2}, \ldots, \mathcal{M}_{8}$.

6.1. Es gibt eine leere Menge (wegen $\mathcal{M} \neq \emptyset, 5.2(\mathrm{~b})$ und (2)).

6.2. Es gibt eine nichtleere Menge (wegen $\mathcal{M} \neq \emptyset$ und (4)).

6.3. Für Teilmengen $A, B$ einer Menge ist die Aussage $A=B$ definit [(1)(4)].

6.4. Oben spielt die $\underline{\text { Klasse }} \mathcal{M}$ nirgends eine wesentliche Rolle. Es genügt, von "Mengen" reden zu können (lies $A \in \mathcal{M}$ wie $x \in \mathcal{D}$ ). Wie wichtig aber $\mathcal{M}$ als Klasse ist, erweist sich an einem einfachen Beispiel:

Gemäß 5.6' existiert $\gamma: \mathcal{M} \rightarrow \mathcal{M}$ mit $X \gamma=\mathcal{F}(X)$, und $\gamma$ darf in jedem Existenzbeweis vorausgesetzt werden.

Wende nun 5.6 an mit $\mathcal{M}$ anstelle $A$ und $\gamma$ anstelle $\beta$, sowie der Eigenschaft $E$ von $X \in A$ und $y \in X \beta$, daß $y$ "die" identische Abbildung von $X$ ist (laut 5.8(b) existiert zu $X$ immer ein $y$ ).

Das Resultat ist die Existenz der Funktion $\alpha: \mathcal{M} \rightarrow \gamma$, gewöhnlich mit id bezeichnet, welche jeder Menge $X$ die identische Abbildung id ${ }_{X}$ von $X$ zuordnet. Sie steht in jedem Standardbeweis zur Verfügung.

Nebenbei haben wir die typische Art und Weise kennengelernt, wie 5.6' und $5.7^{\prime}$ angewandt werden, nämlich mit $B=\mathcal{M}$.

6.5. In (7) wie in (8) würde die Existenz von $A$ und $\alpha$ genügen, wenn die Aussage $K \in \mathcal{M}$ stabil wäre. So reicht es nur für $(K \in \mathcal{M})^{f f}$.

Mit 1.5(a) bzw. 6.2 und 5.3 folgt

6.6 $6_{f f} K \in \mathcal{K}$ ist eine Menge wenn $A$ und $\alpha$ wie in $\mathcal{M}_{7}$ oder $\mathcal{M}_{8}$ existieren.

$6.7_{f f}$ Jede einelementige Klasse ist eine Menge.

Der Zusatz $f f$ deutet an, daß nicht die zur Schau gestellte Aussage $S$, hier 6.6 bzw. 6.7, behauptet wird, sondern nur ihr stabiler Abschluß $S^{f f}$.

$K$ einelementig bedeutet neben $K \neq \emptyset$ noch $x=y$ für alle $x, y \in K$.

Ein $\alpha: A \rightarrow K$ ist surjektiv wenn $A \neq \emptyset$ und $K$ einelementig ist. 


\section{Starke Existenz.}

7.1. Das übliche „Definieren“ von Mengen und Abbildungen hat zuerst Zermelo als fragwürdig erkannt und in einem gewissen Sinne begründet [20].

Neben Begrifflichem war eine solche Begründung auch unser bisheriges Hauptanliegen. Jetzt folgt die Bewältigung der Probleme, welche die Aufgabe des obskuren, mit einem naiv-gegenständlichen Objektbegriff einhergehenden Begriffes „Identität“ nach sich zieht.

Im wesentlichen geht es um die Gewohnheit, einem eindeutig existierenden Objekt nicht nur einen Namen wie $a, b, c$ zu geben, sondern einen wie etwa $\mathrm{id}_{K}$, $A \cap B, \alpha \beta$, der die Namen von (prinzipiell allen) Objekten involviert, welche der Existenz zugrunde liegen; der also eine gewisse „funktionale“ Abhängigkeit erkennen läßt. Einen Lösungsweg haben wir soeben anhand $\mathrm{id}_{K}$ kennengelernt. Er wird in $\S 8$ weiterverfolgt und entspricht dem im frühen 20. Jahrhundert vollzogenen Übergang von „Funktionen“ alten Sinnes zu Abbildungen im modernen Sinne Dedekinds.

Jetzt setzen wir zwei Funktionen voraus, $\wp$ und $\ell$, die jene Gewohnheit und mit ihr sogar die ganze r. e. M. auf das vollkommenste fundieren.

Axiom $\mathbf{I}^{+}$. $\wp$ ist eine 2-variable Funktion, welche jedem Bereich $A$ und jeder Aussage $S$ einen Teilbereich $S A \wp$ von $A$ zuordnet mit

$$
x \in A \Longrightarrow x \in S A \wp \Leftrightarrow S .
$$

Axiom $\mathrm{II}^{+}$. Für jede einelementige Klasse $A$ gilt $\ell(A) \in A$.

Axiom $\mathrm{I}^{+}$erfordert noch eine Voraussetzung: Jede Aussage ist ein Objekt. ${ }^{57}$

Und $\mathrm{I}^{+}$impliziert I: wende dort $\mathrm{I}^{+}$mit $x \in A \wedge E$ anstelle $S$ an. Wesentlich ist allein, daß $U$ in Axiom I jetzt explizit gegeben ist, etwa als $\{x \in A \mid E\}$, und nicht nur existiert.

Die gleich auftretenden vertrauten Bezeichnungen stellen nur eine harmlose Umbenennung des ursprünglich durch $\wp$ und $\ell$ gegebenen dar.

Sich so darstellen zu lassen - via $\wp$ und $\ell$ - ist das Wesen „starker" Existenz, allgemeiner überhaupt die Möglichkeit einer expliziten Darstellung. Vergleiche mit dem Begriff „konstruierbar“ in der r. e. M. ${ }^{58}$

\footnotetext{
${ }^{57}$ Das erlaubt, den eigenständigen Grundbegriff eines Bereiches zu eliminieren:
}

Verstehe unter einem Bereich eine Abbildung $A$ der Art, daß $x A$ immer (für jedes Objekt $x$ ) eine Aussage ist. Schreibe $x \in A$ statt $x A$.

Außerdem läßt sich 1.7(a)(b) nun so ausdrücken:

Es gibt keine indefinite und keine instabile Aussage.

${ }^{58}$ Von Artin stammt folgendes Prinzip: Wenn es möglich ist, durch begriffliches Schließen die Einzigkeit eines mathematischen Gegenstandes zu beweisen, so ist es auch möglich, ihn explicite formelmäßig anzugeben [H. Zassenhaus: Methoden und Probleme der modernen Algebra. Jb. d. Dt. Math.-Verein. 96(1994)]. 
So wie Axiom I wird $\mathrm{I}^{+}$auf den Grundbereich einer Klasse $K$ angewandt. Das führt zu folgendem Analogon von 5.4:

7.2. Starke Existenz von Teilklassen.

Sei $K$ eine Klasse und $E$ eine $K$-Aussage über $x \in K$ [5.3].

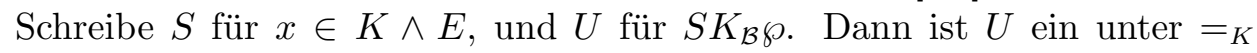
abgeschlossener Teilbereich von $K$ mit $x \in K \Longrightarrow x \in U \Leftrightarrow E$.

Bezeichne die Teilklasse $U /=_{K}$ mit $\{x \in K \mid E\}$.

7.3. Daraus resultieren die folgenden Teilklassen einer Klasse $K$ : das ,Komplement" $K \backslash A=\{x \in K \mid x \notin A\}$ einer Teilklasse $A$, Durchschnitt $A \cap B$ und Vereinigung $A \cup B$ von Teilklassen $A, B$, Durchschnitt $\bigcap_{\mathcal{A}}$ und Vereinigung $\cup_{\mathcal{A}}$ aller $A \in \mathcal{A}$, wobei $\mathcal{A} \subseteq \mathcal{P}(K)$, der Durchschnitt $\langle X\rangle_{\mathcal{A}}$ aller $A \supseteq X$ in $\mathcal{A}$.

Letzteres ist hauptsächlich dann von Interesse, wenn $\mathcal{A}$ schnittabgeschlossen ist, d. h. jeder Schnitt von Elementen von $\mathcal{A}$ liegt wieder in $\mathcal{A}$. Dann ist $\langle X\rangle_{\mathcal{A}}$ das $\mathcal{A}$-Erzeugnis von $X$. Ein schönes Beispiel findet sich in I.5.8.

Der Schnitt $A \cap B$ ist auch für Klassen $A, B$ von Klassen gegeben, und zwar als Teilklasse von $A$ wie auch von $B$.

Beispiel: Die Klasse $\mathcal{P}_{\mathcal{M}}(K)=\mathcal{P}(K) \cap \mathcal{M}$ aller Teilmengen von $K$.

\section{4. $\mathrm{Zu} \alpha: A \rightarrow B$ gehören}

das Urbild $Y \alpha^{-1} \subseteq A$ von $Y \subseteq B$, bestehend aus den $x \in A$ mit $x \alpha \in Y$, das (volle) Urbild $y \alpha^{-1} \subseteq A$ von $y \in B$, bestehend aus den $x \in A$ mit $x \alpha=y$, das Bild $X \alpha \subseteq B$ von $X \subseteq A$, bestehend aus den $y \in B$ mit $y \alpha^{-1} \cap X \neq \emptyset$.

Die Teilklassen $\operatorname{Inj}(A, B), \operatorname{Sur}(A, B), \operatorname{Bij}(A, B)$ von $\mathcal{F}(A, B)$ sollen natürlich aus allen injektiven, surjektiven, bijektiven $\alpha: A \rightarrow B$ bestehen.

7.5. Starke Existenz von Abbildungen mit vorgegebenen Eigenschaften ist eine typische Anwendung von $\ell$. Hauptbeispiele sind

$$
\begin{gathered}
\operatorname{id}_{K}=\ell(\{\alpha \in \mathcal{F}(K) \mid x \alpha=x \text { für alle } x \in K\}) \\
\alpha^{-1}=\ell(\{\beta \in \mathcal{F}(B, A) \mid y \beta \alpha=y \text { für alle } y \in B\}) \\
\alpha \beta=\ell(\{\gamma \in \mathcal{F}(A, C) \mid x \gamma=x \alpha \beta \text { für alle } x \in A\})
\end{gathered}
$$

zu Klassen $K, A, B, C$ sowie $\alpha: A \rightarrow B$ (bijektiv für $\alpha^{-1}$ ), und $\beta: B \rightarrow C$.

Nach $5.7(\mathrm{~b})(\mathrm{c})$ und $5.6^{\prime}$ ist die jeweilige Klasse $\{\ldots\}$ nicht leer.

Des weiteren ist $\emptyset=\ell(\{X \in \mathcal{M} \mid X=\emptyset\})$ die leere Menge.

Und über 5.3 erhalten wir auch noch die konstanten Funktionen

$$
\kappa_{A, B}: x \rightarrow B \quad \text { und } \quad \kappa_{A, B, b}: x \rightarrow b
$$


7.7. Jede einelementige Klasse $K$ ist eine Menge (vgl. mit $6.7_{f f}$ ).

Das folgt aus $\mathcal{M}_{7}$, angewandt mit $A=\mathcal{P}(\emptyset)$ und $\alpha=\kappa_{A, K}$.

7.8. Der Regularitätssatz: Für Elemente $x, y$ und Teilklassen $X, Y$ einer Klasse $K$ sind die Aussagen $x=y, x \in Y, X=Y, X \subseteq Y$ definit.

Kurz: $K$ ist regulär.

Beweis. Da $\{x\}$ eine Menge ist, ist $\{x\} \cap Y=\{x\}[\Leftrightarrow x \in Y]$ definit. Mit $\{y\}$ anstelle $Y$ folgt: $x=y$ ist definit. Mit $\mathcal{P}(K)$ anstelle $K$ folgt: $X=Y$ ist definit. Und wegen $X \subseteq Y \Leftrightarrow X \cap Y=X$ ist schließlich auch $X \subseteq Y$ definit.

7.9. Über den Klassenexistenzsatz [7.2] folgt, daß jede $K$-Aussage $E$ über die Elemente $x$ einer Klasse $K$ definit ist: $x \in K \Rightarrow E \vee E^{f}$.

Auch jede Relation auf einer Klasse ist definit, und die Gänsefüßchen um das Wort Komplement in 1.3 können wir jetzt streichen: jedes $x \in K$ liegt oder liegt nicht in $A$. Somit gilt

$$
K=A \dot{\cup} K \backslash A \quad \text { für } \quad A \subseteq K \in \mathcal{K},
$$

und was definit ist, ist auch stabil [1.6(b)].

7.10. Ich rede vom +- Modus, wenn die starken Axiome $\mathrm{I}^{+}$und $\mathrm{II}^{+}$vorausgesetzt werden; vom $f f$-Modus, wenn alle Aussagen stabil und definit sind, wie in jedem Standardbeweis [1.10]; und ansonsten vom 0-Modus, wenn also allgemein nur $\S 1-6$ vorausgesetzt wird.

Wenn sich nun im +-Modus die Verhältnisse in der r. e. M. tatsächlich „,auf das vollkommenste" widerspiegeln, dann muß auch genau wie im $f f$-Modus ein als existent notiertes Objekt sofort verwertbar sein [3.5]. Es bietet sich daher an, $\mathrm{II}^{+}$allgemein für nichtleeres $A$ vorauszusetzen. Dazu zweierlei:

Erstens ist $\rho=\kappa_{A, A}$ eine Äquivalenzrelation von $A$, die Faktorklasse $A / \rho$ einelementig, und somit ist mit $\ell(A / \rho)$ ein Element von $A$ explizit gegeben.

Zweitens wird aufgrund 7.8 jede halbwegs normale Behauptung stabil sein, der Beweis also im $f f$-Modus stattfinden. In höheren Sphären mag es Ausnahmen geben. Eine solche aus dem Bereich Kardinal- und Ordinalzahlen wollte ich hier eigentlich angeben, darauf beruhend, daß für Klassen $K$ die Aussage $K \in \mathcal{M}$ nicht als stabil vorausgesetzt wurde. Sie ist aber doch stabil (sogar definit), und zwar weil $K$ ein Element und $\mathcal{P}_{\mathcal{M}}(K)=\mathcal{P}(K) \cap \mathcal{M}$ eine Teilklasse der regulären Klasse $\mathcal{P}(K)$ ist. Wir halten fest:

$K \in \mathcal{K} \quad \Longrightarrow \quad \mathcal{K} \in \mathcal{M}$ ist stabil und definit. 


\section{Existenz multivariabler Abbildungen.}

8.1. (a) Ohne die starken Axiome in $\S 7$, besonders $\mathrm{I}^{+}$, liegt jede nicht von vornherein als stabil erkannte Behauptung außer Reichweite, und zwar weil sich im Beweis keinerlei Existenzinformation verwerten läßt [3.5]. In diesem Abschnitt befassen wir uns also mit Standardbeweisen, mit dem $f f$-Modus.

(b) Dem liegt der Eindruck zugrunde, das alles, was wirklich zählt in der Mathematik, was mit einigem Recht den Namen „Satz“ verdient, von Natur aus stabil ist (bei „Hilfssätzen“ $S$ lohnt es sich nicht, über die Stabilität nachzudenken, denn $S^{f f}$ genügt). Das fehlende $\vee$ in 1.6(d) scheint das in Frage zu stellen. Die Essenz eines Odersatzes $S$, etwa $U \Rightarrow T_{1} \vee T_{2}$, liegt jedoch in der Möglichkeit, eine Aussage $U \Rightarrow V$ durch Inspektion der Fälle $T_{i}$ zu beweisen, und dafür genügt $S^{f f}$, vorausgesetzt $U \Rightarrow V$ ist stabil, d.h. $V$ ist stabil auf der Basis von $U$ [1.8]. Bei stabilem $T_{2}$ ist $S^{f f}$ gleichwertig mit $U \Longrightarrow T_{1}^{f} \Rightarrow T_{2}$ [1.5(a)(c)], und durch letzteres wird in der r. e. M. gewöhnlich $T_{1} \vee T_{2}$ bewiesen.

Jeder Klassifikationssatz, wie der über die endlichen einfachen Gruppen, ist ein Beispiel für einen allgemeinen Odersatz $S$ (mit mehreren $T_{i}$, alle stabil).

Zur Erhellung: Daß eine bestimmte Person $P$ sich in Tokio, Chicago, Oslo, oder sich in einem von hundert explizit genannten bayerischen Dörfern aufhält, interessiert, wenn überhaupt, wohl nur insofern, als $P$ jedenfalls (in jedem Falle) nicht in der näheren Umgebung von beispielsweise Hamburg anzutreffen ist.

(c) Um im $f f$-Modus frei von allen möglichen Objekten reden zu können, etwa denen in 7.3/4/5, bedarf es geeigneter Funktionen, wie schon in 6.4 und 7.1 angedeutet. Was auch schon durch 6.4 deutlich wird, ist daß jede „Klasse“ ihre eigene Funktionenwelt hat, während Mengen durchweg variabel sind.

Zum Beispiel wird die zu einer Klasse $K$ gehörige Schnittfunktion $\cap$ je zwei Teilklassen $A, B$ den Schnitt $A \cap B \in \mathcal{P}(K)$ zuordnen, während auf der Mengenebene auch $K(\in \mathcal{M})$ variabel ist.

(d) Deutlichkeitshalber könnte man $A \cap_{K} B$ schreiben. Eine solche, auch bei anderen Beispielen oft bequeme Bezeichnungsweise kann dazu verleiten, auch auf der Klassenebene frei von $A \cap_{K} B$ (oder doch wieder kurz $A \cap B$ ) zu reden. Das ist auch ganz in Ordnung solange nur einzelne „Klassen“ $K$ involviert sind, denn grundsätzlich ist bei jedem $K$ explizit die Existenz der Schnittfunktion (zunächst etwa $\sigma$, dann in $\cap$ oder $\cap_{K}$ umbenannt) zu konstatieren, um dann 3.5 anwenden zu können.

(e) Soweit später ohne weitere Begründung von dieser oder jener Funktion die Rede ist, von ihr Gebrauch gemacht wird, ergibt sich ihre Existenz sofort aus 5.6, manchmal verbunden mit 5.6', 5.7, 5.7'. Immer darauf zu verweisen, wäre einfach zu langweilig (gelegentlich tue ich es aber doch).

Entsprechendes gilt erst recht für die Existenz von Teilklassen via 5.4. 


\subsection{Die Existenz von $\cap_{K}$ :}

(1) $\mathrm{Zu} A \subseteq K$, existiert $\alpha_{1}: \mathcal{P}(K) \rightarrow \mathcal{P}(K)$ mit

$$
B \alpha_{1}=\{y \in K \mid y \in A \wedge y \in B\} .
$$

(2) Es gibt ein $\alpha_{2}: \mathcal{P}(K) \rightarrow \mathcal{F}(\mathcal{P}(K))$ der Art, daß $A \alpha_{2}$ wie $\alpha_{1}$ in (1) ist.

Dann ist $\alpha_{2}$ die angestrebte Function $\cap_{K}$.

Für Mengen $K$ wird in einem weiteren Schritt (3) noch die Existenz einer Abbildung $\alpha_{3}: \mathcal{M} \rightarrow$ ?, konstatiert, welche jedem $K$ ein $\alpha_{2}$ wie in (2) zuordnet. Nun liegt $\alpha_{2}$ aber in $F=\mathcal{F}(\mathcal{P}(K), \mathcal{F}(\mathcal{P}(K)))$. Deshalb wird genau wie in 6.4 die $\beta$-Version von 5.6 benötigt $(\beta=$ ?), während für (1) and (2) die $B$-Version reichte. Wir brauchen also die Abbildung $\beta: \mathcal{M} \rightarrow \mathcal{M}$ mit $K \beta=F$. Sie existiert aufgrund 5.6', wieder mit $\mathcal{M}$ anstelle $B$ angewandt.

Die Bezeichnung $\alpha_{1}, \alpha_{2}, \alpha_{3}$ deutet eine gewisse Systematik an. In ihrem Sinne wäre auch $\beta_{3}$ anstelle $\beta$, und (n) würde (für $\mathrm{n}>1$ ) immer so aussehen:

(n) $\exists \alpha_{n}: A_{n} \rightarrow \beta_{n}$ der Art, daß $x \alpha_{n}$ immer wie $\alpha_{n-1}$ in (n-1) ist.

Ich empfehle das Beispiel $X \alpha$ : Zu kreieren ist eine 4-variable Funktion $\mu$, welche Mengen $A, B$ sowie $\alpha: A \rightarrow B$ und $X \subseteq A$ das Bild $X \alpha \subseteq B$ zuordnet.

Ich schlage auch noch das Kompositum $\alpha \beta: A \rightarrow C$ vor, eine 5 -variable Funktion soll es Mengen $A, B, C$ sowie $\alpha: A \rightarrow B$ und $\beta: B \rightarrow C$ zuordnen.

8.3. Ich selbst werde das Thema so nicht weiterverfolgen. Es gibt nämlich eine Alternative zu obigem Vorgehen, und zwar mit Abbildungen auf geeigneten Produktklassen (pc-Funktionen) zu arbeiten statt mit multivariablen Abbildungen im bisherigen Schönfinkelschen Sinne (sf-Funktionen). Damit wird $\cap_{K}: \mathcal{P}(K) \times \mathcal{P}(K) \rightarrow \mathcal{P}(K)$ in einem Schritt ,definiert", via 5.7.

Für Mengen $K$ (variabel) braucht man die Klasse $P=\mathcal{M} \times \mathcal{P} \times \gamma$ aller Tripel $(K, A, B)$, also mit $\gamma: \mathcal{M} \times \mathcal{P} \rightarrow \mathcal{M},(K, A) \gamma=\mathcal{P}(K)$, existent aufgrund 5.7'.

Wende 5.7 dann mit $(K, A, B) \beta=K$ an. Erhalte $\sigma[=\cap]$.

Ein einfacheres Beispiel: die Komplementfunktion $\mathcal{M} \times \mathcal{P} \rightarrow \gamma, \gamma$ wie eben.

8.4. Jetzt erledigen wir die pc-Version der ersten Aufgabe.

Die aus den Quadrupeln $(A, B, \alpha, X)$ bestehende Produktklasse

$$
P=\mathcal{M} \times \mathcal{M} \times \gamma_{1} \times \gamma_{2}
$$

erfordert $\gamma_{1}: \mathcal{M} \times \mathcal{M} \rightarrow \mathcal{M}$ mit $(A, B) \gamma_{1}=\mathcal{F}(A, B)$ sowie $\gamma_{2}: \mathcal{M} \times \mathcal{M} \times \gamma_{1} \rightarrow \mathcal{M}$ mit $(A, B, \alpha) \gamma_{2}=\mathcal{P}(A)$.

Dann wird noch $\beta: P \rightarrow \mathcal{M}$ mit $(A, B, \alpha, X) \beta=B$ gebraucht.

Existenz jeweils aufgrund 5.7'. Aus 5.7 ergibt sich dann $\sigma: P \rightarrow \mathcal{P}(B)$ mit $(A, B, \alpha, X) \sigma=\left\{y \in B \mid \exists_{x \in X} x \alpha=y\right\}$, und $\sigma$ ist das erstrebte $\mu$.

Variante: Arbeite mit $(\ldots) \beta=\mathcal{P}(B)$. Gewinne $\alpha: P \rightarrow \beta$ aus 5.7. 
8.5. Objekten $x_{1}, x_{2}, x_{3}, \ldots, x_{n}$ einer gewissen Art soll mittels einer pcFunktion $\mu$ ein Objekt $y$ einer gewissen Art zugeordnet werden.

Das Existenzproblem für $\mu$ wird jetzt in einem gewissen Rahmen gelöst.

Es handelt sich nur um eine meta-mathematische Betrachtung. Sie erklärt, wie die Aufgabe sich im Einzelfall routinemäßig erledigen läßt. Setze voraus:

(1) eine Klasse $K_{1}$ und eine Eigenschaft $E_{1}$ von $x_{1} \in K_{1}$,

(2) zu $x_{1}$ mit $E_{1}$ eine explizit gegebene Klasse $K_{2}=f_{1}\left(x_{1}\right)$,

$(2)^{\prime}$ eine Eigenschaft $E_{2}$ von $x_{1}\left(\right.$ mit $\left.E_{1}\right)$ und $x_{2} \in K_{2}$,

(3) zu $x_{1}, x_{2}$ mit $E_{1}, E_{2}$ eine explizit gegebene Klasse $K_{3}=f_{2}\left(x_{1}, x_{2}\right)$,

$(3)^{\prime}$ eine Eigenschaft $E_{3}$ von $x_{1}, x_{2}\left(\right.$ mit $\left.E_{1}, E_{2}\right)$ und $x_{3} \in K_{3}$,

$(\mathrm{n}+1)$ zu $x_{1}, \ldots, x_{n}$ mit $E_{1}, \ldots, E_{n}$ eine e. g. Klasse $K_{n+1}=f_{n}\left(x_{1}, \ldots, x_{n}\right)$, $(\mathrm{n}+1)^{\prime}$ eine Eig. $E_{n+1}$ von $x_{1}, \ldots, x_{n}\left(\operatorname{mit} E_{1}, \ldots, E_{n}\right)$ und $x_{n+1} \in K_{n+1}$.

Für jedes $i$ sei $f_{i}$ konstant oder $f_{i}(\ldots)$ eine Menge.

Und selbstverständlich sollen die $E_{i}$ und $f_{i}$ mit $=$ verträglich sein.

Die $y$ 's sind die $x_{n+1}$ mit $E_{n+1}$, und deshalb wird noch vorausgesetzt, daß zu $x_{1}, \ldots, x_{n}$ mit $E_{1}, \ldots, E_{n}$ immer ein solches $y$ existiert.

Nach 5.4 existiert $K=\left\{x_{1} \in K_{1} \mid E_{1}\right\}$.

Dann wird die „Pseudofunktion“ $f_{1}$ in eine richtige Funktion $\beta_{1}$ umgemünzt: Via 5.3 wenn $f_{1}$ konstant ist, und sonst via $5.6^{\prime}$ (wieder mit $B=\mathcal{M}$ ).

Auf $\beta_{1}$ läßt sich dann 5.6 anwenden, und wir erhalten

$$
\sigma_{1}: K \rightarrow \mathcal{K} \text { mit } x_{1} \sigma_{1}=\left\{x_{2} \in K_{2} \mid E_{2}\right\}
$$

Ersetze analog $f_{2}$ durch $\beta_{2}$ und erhalte, jetzt via 5.7,

$$
\sigma_{2}: K \times \sigma_{1} \rightarrow \mathcal{K} \operatorname{mit}\left(x_{1}, x_{2}\right) \sigma_{2}=\left\{x_{3} \in K_{3} \mid E_{3}\right\} .
$$

So fortfahrend, gelangen wir schließlich $\mathrm{zu}$ $\sigma_{n}: K \times \sigma_{1} \times \sigma_{2} \times \ldots \times \sigma_{n-1} \rightarrow \mathcal{K} \operatorname{mit}\left(x_{1}, \ldots, x_{n}\right) \sigma_{n}=\left\{x_{n+1} \in K_{n+1} \mid E_{n+1}\right\}$.

Eine Auswahlfunktion zu $\sigma_{n}$ ist dann das erstrebte $\mu$.

8.6. Die Daten des Beispiels $X \alpha$ in 8.4 (Variante):

$$
\begin{aligned}
& K_{1}=\mathcal{M}=K \text { und } f_{1}(A)=\mathcal{M}=K_{2} \\
& f_{2}(A, B)=\mathcal{F}(A, B)=K_{3} \\
& f_{3}(A, B, \alpha)=\mathcal{P}(A)=K_{4} \\
& f_{4}(A, B, \alpha, X)=\mathcal{P}(B)=K_{5} \\
& E_{5}(A, B, \alpha, X, Y) \Longleftrightarrow Y=\left\{y \in K_{5} \mid \exists_{x \in X} x \alpha=y\right\}
\end{aligned}
$$

$\mathrm{Zu} A, B, \alpha, X$ existiert ein $Y$ mit $E_{5}$ aufgrund 5.4. 
Ein etwas komplexeres Beispiel ist der Fortsetzungssatz [5.8(d)] für Mengen. Das Ziel ist jetzt eine 6-variable Funktion $\mu$. Sie soll Mengen $A, B$ und weiter $U, \lambda, U^{\prime}, \lambda^{\prime}$ eine (die) Fortsetzung $\alpha$ zuorden ( $\alpha$ existiert).

Wie eben werden triviale, d.h. nicht einschränkende $E_{i}$ 's unterdrückt.

$$
\begin{aligned}
& K_{1}=\mathcal{M}=K \text { und } f_{1}(A)=\mathcal{M}=K_{2} \\
& f_{2}(A, B)=\mathcal{P}(A)=K_{3} \\
& f_{3}(A, B, U)=\mathcal{F}(U, B)=K_{4} \\
& f_{4}(A, B, U, \lambda)=\mathcal{P}(A)=K_{5} \\
& E_{5}\left(A, B, U, \lambda, U^{\prime}\right) \Longleftrightarrow A=U \dot{\cup} U^{\prime} \\
& f_{5}\left(A, B, U, \lambda, U^{\prime}\right)=\mathcal{F}\left(U^{\prime}, B\right)=K_{6} \\
& f_{6}\left(A, B, U, \lambda, U^{\prime}, \lambda^{\prime}\right)=\mathcal{F}(A, B) \\
& E_{7}\left(A, B, U, \lambda, U^{\prime}, \lambda^{\prime}, \alpha\right) \Longleftrightarrow \alpha=\lambda \text { auf } U \text { und } \alpha=\lambda^{\prime} \text { auf } U^{\prime}
\end{aligned}
$$

Um die beiden Aufgaben in 8.2 zu vereinfachen, kann man die involvierten Mengen in der Produktklasse $\mathcal{M} \times \mathcal{M}$ bzw. $\mathcal{M} \times \mathcal{M} \times \mathcal{M}$ zu jeweils einem Objekt verschmelzen und so die Variablenzahl auf 3 reduzieren.

Analog läßt sich die Situation in 8.5 vereinfachen, indem man alle konstanten $K_{i}$ (mitsamt $K_{1}$ ) zu einem neuen $K_{1}$ zusammenfaßt. Danach ist $f_{i}(\ldots)$ immer eine Menge. Das könnte bei einer allgemeineren Aufgabe von Nutzen sein: Versuche eine pc-Funktion (etwa $\mu$ in 8.5) in eine sf-Funktion zu transformieren. Der inverse Prozeß ergibt sich sofort aus 5.7' , dazu muß natürlich schon eine Produktklasse vorliegen.

8.7. Jede funktional formulierte Namensgebung für ein Objekt läuft auf die Existenz einer entsprechenden (multivariablen) Funktion hinaus, welche dann im $f f$-Modus in dem jeweiligen Rahmen dieses Objekt zur Verfügung stellt.

Nach einigen exemplarischen Erfahrungen einfach auf $8.5 \mathrm{zu}$ vertrauen, würde ich durchaus als eine natürliche Haltung ansehen. Auch hängt die Masse der ständig in alles mögliche hineinwirkenden Objekte, wie die in $7.2 / 3 / 4,{ }^{59}$ nur von wenigen Parametern ab, und für die Existenz der entsprechenden Funktionen gilt das in 8.1(e) gesagte.

Und so wie die fraglichen Definitionen in der r.e.M. aufeinander aufbauen, so auch die sie fundierenden Funktionen. Es treten jeweils nur wenige neue Parameter auf. Das Denken wird also nach einer gewissen Gewöhnung mehr von 8.1(e) beherrscht werden als von 8.5.

\footnotetext{
${ }^{59}$ oder das Einselement 1 einer Gruppe $G$, oder das Inverse $x^{-1}$ eines Elementes $x$, oder der Normalisator $N_{G}(U)$ einer Untergruppe $U$,
} 
Die beiden Beispiele in 8.6 machen etwas anderes deutlich:

Je komplexer eine multivariable Funktion, desto unbedeutender ist sie:

Während $X \alpha$ alles durchdringt und frei verfügbar sein muß, wird in aller Regel die reine Existenz einer Fortsetzung genügen. In der r. e. M. redet man einfach von „der Fortsetzung $\alpha$ " oder man sagt „, es gibt (genau) eine Fortsetzung $\alpha$ ", was auf dasselbe hinausläuft. Und es gibt keinen Grund, sich hier anders zu verhalten. Außer man arbeitet auf einem Feld, wo man ständig mit Fortsetzungen zu hantieren hat und deshalb passende Namen einführen möchte.

Der Ordnung halber erwähne ich noch, daß eine Existenzaussage für eine multivariable pc-Funktion implizit immer die Existenz der zugrundeliegenden Produktklasse, d.h. ihrer Komponenten, einschließt.

8.8. Bevor der Beweis einer stabilen Aussage $S$ beginnt, muß $S$ erst einmal formuliert werden, und dafür steht im 0-Modus nur die in $\S 1-6$ entwickelte Grundprache zur Verfügung. Als Lösung dieses Problem sehe ich die in 5.2(d) formulierte Konvention (kurz $\emptyset$-Konvention); sie soll natürlich auch für Begriffe gelten, die aus jenen lediglich existenten Objekten resultieren. Dazu gehören, wie wir gleich sehen werden, Begriffe wie Gruppe, Körper, topologischer Raum.

Besonders bequem und für Klarheit sorgend ist die Vorstellung, daß unser ganzes Tun einem zukünftigen Standardbeweis dienen soll.

Dann sind wir immer im $f f$-Modus. Beachte auch die nächste Fußnote.

8.9. Als Beispiel formuliere ich den $f$-Kettensatz I.5.5 neu.

Für Klassen, als Existenzsatz, inklusive Zusatz, in der "Grundsprache“.

Der allgemeine $f$-Kettensatz:

Sei $M$ eine Klasse, $\mathcal{A} \subseteq \mathcal{P}(M), f: \mathcal{A} \rightarrow M$ und $f(A) \notin A$ für $A \in \mathcal{A}$.

Nenne $W \in \mathcal{P}(M)$ eine $f$-Kette, wenn es eine Wohlordnung $\leq$ auf $W$ gibt, derart daß jeder Abschnitt $A \neq W$ von $W$ in $\mathcal{A}$ liegt, mit $f(A) \in W$ und $f(A) \leq x$ für alle $x \in W$ mit $x \notin A$.

Dann existiert eine $f$-Kette $V \notin \mathcal{A}$ der Art, daß die $f$-Ketten genau die Abschnitte von $V$ sind, und zwar bezüglich jeder $f$-Ordnung.

Beim $f$-Kettensatz für geordnete Klassen liegt noch eine Ordnungsrelation $\leq$ auf $M$ vor, $f(A)$ ist immer eine obere Schranke von $A$, und die Ordnung einer $f$-Kette ist die durch $\leq$ gegebene.

In Zukunft werde ich mich doch lieber an die $\emptyset$-Konvention halten, bessere Verständlichkeit einer formal-oberflächlich größeren Präzision vorziehen.

„In Zukunft": Ich werde also weiter im 0-Modus arbeiten, d.h. mit einem Minimum an allgemeinen Voraussetzungen, und auf den + -Modus nur hin und wieder am Rande eingehen. Wer die Dinge lieber in diesem sehen will, möge ein Auge darauf haben, daß „Existenz" immer auf „starke Existenz" hinausläuft. 


\section{Mathematische Strukturen.}

Gegenstand der Mathematik sind mathematische Strukturen.

Einige Beispiele erklären, wie die diversen Typen, genauer Klassen, solcher Strukturen Schritt für Schritt entstehen.

Der einzelne Schritt sieht immer so aus: Liegt eine solche Klasse $\mathcal{S}$ vor und eine Funktion $\rho: \mathcal{S} \rightarrow \mathcal{M}$, so ist $\mathcal{S} \times \rho$ eine neue Klasse mathematischer Strukturen, wie dann auch jede Teilklasse.

Wir beginnen mit $\mathcal{S}=\mathcal{M}$ und den Funktionen Op und Rel, die einer Menge $M$ die Mengen $\mathcal{F}(M \times M, M)$ und $\mathcal{F}(M, \mathcal{P}(M))$ aller zweistelligen Operationen (Verknüpfungen) bzw. aller Relationen auf $M$ zuordnen.

Sie existieren aufgrund 5.6 und stehen im +- wie im $f f$-Modus sofort zur Verfügung. So auch jede gemäß 5.4 explizit angegebene Teilklasse. Das führt beispielsweise zur Klasse $\mathcal{G}$ aller Gruppen, einer Teilklasse von $\mathcal{M} \times$ Op.

Genausogut könnte man $\mathcal{G}=\mathcal{M} \times \mathrm{Op}_{g r}$ definieren, wobei die Funktion $\mathrm{Op}_{g r}$ jedem $M$ die Menge aller Gruppenoperationen auf $M$ zuordnet.

Die wichtigste Teilklasse von $\mathcal{M} \times \operatorname{Rel}$ (Klasse der gerichteten Graphen) ist die Klasse der geordneten Mengen (Geomengen). Es folgt die Klasse $\mathcal{W}$ der wohlgeordneten Mengen (Womengen, Ketten). Die Klasse der topologischen Räume ist eine Teilklasse von $\mathcal{M} \times \mathcal{P}^{2}$, mit $\mathcal{P}^{2}(M)=\mathcal{P}(\mathcal{P}(M))$.

Auf einer gegebenen Teilklasse $\mathcal{A}$ von $\mathcal{M} \times \rho$, mit $\rho: \mathcal{M} \rightarrow \mathcal{M}$, wird $\mathrm{Op}^{\prime}(A)=\mathrm{Op}\left(A_{1}\right)\left[=\mathrm{Op}\left(A \pi_{1}\right)\right]$ definiert und analog $\operatorname{Rel}^{\prime}(A)=\operatorname{Rel}\left(A_{1}\right)$.

Aus der Klasse $\mathcal{A}$ der abelschen Gruppen entsteht so die Klasse aller Körper, eine Teilklasse von $\mathcal{A} \times \mathrm{Op}^{\prime}$, sowie die Klasse aller geordneten Gruppen, eine Teilklasse von $\mathcal{A} \times \operatorname{Rel}^{\prime}$.

Im Vordergrund stehen Strukturen mit einer Grundmenge, die sich also wie oben aus $\mathcal{M}$ entwickeln. Dies könnte auch in Abhängigkeit von gewissen vorgegebenen Objekten geschehen, wie bei Moduln, insbesondere Vektorräumen.

Man kann aber beispielsweise auch von $\mathcal{M} \times \mathcal{M}$ ausgehen.

Auf der Klassenebene läßt sich obiges nicht nachvollziehen, es fehlt das Analogon zu $\mathcal{M}$, die Klasse aller Klassen.

Es läßt sich aber von einzelnen Klassen und einzelnen Verknüpfungen sowie Relationen reden, unter Verwendung der auf der Mengenebene entwickelten Terminologie. Wenn beispielweise von einer geordneten Klasse (Geoklasse) $K$ die Rede ist, so soll damit nur angedeutet sein, daß zu der Klasse $K$ auch noch eine Ordnungsrelation $\leq$ gegeben ist. Und bei einer Pseudogruppe $G$ ist der Klasse $G$ noch eine Gruppenmultiplikation beigefügt. Ihre „Untergruppen“ sind Teilklassen mit gewissen Eigenschaften, Untergruppen einer "richtigen“ Gruppe (Element von $\mathcal{G}$ ) dagegen Gruppen mit gewissen Eigenschaften. 


\section{Kardinal- und Ordinalzahlen.}

10.1. Das Thema gehört in einen allgemeineren Zusammenhang.

Jede Klasse $\mathcal{S}$ mathematischer Strukturen geht einher mit dem Begriff eines Isomorphismus und dem einer Teilstruktur, wofür es durchaus verschiedene Möglichkeiten gibt. Die Isomorphie führt zu einer Äquivalenzrelation $\simeq$ auf $\mathcal{S}$, und dann existiert eine Klasse $K$ (die Faktorklasse $\mathcal{S} / \simeq$ oder das Bild $\mathcal{S} \simeq$ in $\mathcal{P}(\mathcal{S}))$ mit einer $A \simeq B \Leftrightarrow A \chi=B \chi$ genügenden Surjektion $\chi: \mathcal{S} \rightarrow K .{ }^{60}$

Des weiteren existiert ein Linksinverses $\chi^{\prime}: K \rightarrow \mathcal{S}$.

Den Ursprung von $K$ sollte man zwecks Konzentration auf das wesentliche, die Essenz der axiomatischen Methode, gleich wieder vergessen. Im + -Modus aber erst, nachdem $K$ als stark existent (wie alles andere) registriert wurde.

Schreibe $A \nearrow B$ wenn $A$ zu einer Teilstruktur von $B$ isomorph ist. Die Relation $\nearrow$ existiert und führt zu einer reflexiven und transitiven, aber nicht unbedingt anti-symmetrischen Relation $\leq$ auf $K$ mit $A \nearrow B \Leftrightarrow A \chi \leq B \chi$.

10.2. Wir betrachten zwei Beispiele mit folgenden Eigenschaften:

(a) $\leq$ ist eine Wohlordnung von $K$,

(b) $K$ ist unendlich ohne Maximum,

(c) für jedes $x \in K$ ist der (untere) Abschnitt $x \geq$ eine Menge,

(d) beschränkte Teilklassen und echte Abschnitte von $K$ sind Mengen,

(e) $K$ ist keine Menge.

10.3. Das erste Beispiel ist $\mathcal{S}=\mathcal{M}$, mit den bijektiven Abbildungen und Teilmengen als Isomorphismen und Teilstrukturen. Die vertraute Notation $|A|=|B|$ und $|A| \leq|B|$ steht also für $A \chi=B \chi$ und $A \chi \leq B \chi$.

$\mathrm{Da} \leq \leq$ auch noch anti-symmetrisch ist, und damit eine Ordnungsrelation, ist genau der Inhalt des Satzes von Schröder-Berstein [I.6.6]. Um ihn wie um andere Resultate sowie Notation aus Teil I kümmern wir uns später (in $\S 11$ ).

$\mathcal{C}$ steht für $K$, und die Elemente von $K$ heißen Kardinalzahlen.

$A \chi$ ist die Kardinalität von $A$, und $x \chi^{\prime}$ ist eine Menge mit Kardinalität $x$.

10.4. Im zweiten Beispiel ist $\mathcal{S}$ die Klasse $\mathcal{W}$ der wohlgeordneten Mengen, mit den (unteren) Abschnitten als Teilstrukturen. Die neue Notation für $K, \chi, \chi^{\prime}$ ist $\mathcal{O}, \omega, \omega^{\prime}$, und die Elemente von $K=\mathcal{O}$ heißen Ordinalzahlen.

Dank Cantors Isomorphiesatz I.6.4 ist $\leq$ eine lineare Ordnungsrelation, und zusammen mit Zermelos Wohlordnungssatz I.5.7 erledigt er auch die Linearität der Woklasse $\mathcal{C}[$ I.6.5(a)].

\footnotetext{
${ }^{60}$ Implizit ist von einer regulären Klasse die Rede, und zwar im 0-Modus bei jeder Klassenexistenzaussage.

Allgemeiner existiert, wenn $x$ ein existentes Objekt ist, ein ebensolches Objekt mit der Gratis-Zusatzeigenschaft, daß alle Aussagen stabil und definit sind.

Beispielsweise existiert eine reguläre Klasse $\mathcal{M}^{\prime}=\mathcal{M}$.
} 
10.5. Im Hintergrund (im 0-Modus) stehen zwei Existenzsätze, die jeweils die Existenz einer (regulären) Klasse mit gewissen Eigenschaften behaupten.

$\mathrm{Zu}$ diesen Eigenschaften zählt die Existenz weiterer Objekte $\left(\leq, \chi, \chi^{\prime}\right)$ mit gewissen Eigenschaften. Im Hinblick auf (c) könnte man auch die zu $\leq$ duale Relation $\geq$ einbeziehen, um nicht auf die $\emptyset$-Konvention angewiesen zu sein.

Der Beweis findet dann im $f f$-Modus statt, wo alles stabil und definit ist, insbesondere jede Klasse regulär. ${ }^{61}$ Und wo neben $\geq$ auch die Relationen $<$ und $>$ verfügbar sind, sowie die vier Funktionen, welche jeder Teilklasse $L$ die auf $L$ induzierten Relationen $\leq_{L}, \geq_{L},<_{L},>_{L}$ zuordnen.

Im Falle einer Menge $L$ ist das Paar $\left(L, \leq_{L}\right) \in \mathcal{M} \times$ Rel eine "richtige" geordnete Menge. Und in diesem Sinne können wir im Zuasammenhang mit (c) von den geordneten, sogar wohlgeordneten Mengen $x \geq$ und $x>$ sprechen.

Wir brauchen auch noch die Nachfolgerfunktion $x \rightarrow x^{+}$einer Woklasse [I.5.2]. Sie macht $K$ unendlich wenn es kein Maximum gibt.

Und wir brauchen die Funktionen $\mathcal{A}, \mathcal{A}^{*}: \mathcal{W} \rightarrow \mathcal{W}$ [I.5.1].

Die bezüglich $\subseteq$ wohlgeordnete Menge $\mathcal{A}^{*}(W)$ ist isomorph zu $W$ [I.5.2].

Der angesprochene $\mathcal{O}$-Satz enthält noch eine weitere Behauptung:

(f) Für $W \in \mathcal{W}$ und $x=W \omega$ ist $W \simeq x>$.

Hilfssatz: Sei $A$ eine Woklasse und $\alpha$ eine homomorphe Surjektion auf eine Geoklasse $B\left[a \leq a^{\prime} \Rightarrow a \alpha \leq a^{\prime} \alpha\right]$. Dann ist auch $B$ wohlgeordnet. ${ }^{62}$

Beweis: Sei $Y$ eine nichtleere Teilklasse von $B$ und $X=Y \alpha^{-1}$ ihr Urbild. Dieses hat ein Minimum $x$, und dann ist $x \alpha$ minimal in $Y$.

10.6. Im Falle $K=\mathcal{C}$ folgt (c) so: $x \geq$ ist das Bild $M \chi$ der Menge $M=$ $\mathcal{P}\left(x \chi^{\prime}\right)$ unter $\chi$, ist also nach $\mathcal{M}_{7}$ eine Menge.

Analog ist $x \geq$ im Falle $K=\mathcal{O}$ als homomorphes Bild $M \omega$ der Womenge $M=\mathcal{A}\left(x \omega^{\prime}\right)$ unter $\omega$ sogar eine Womenge.

Und es gibt noch eine Besonderheit, mit $W$ und $x$ wie in $(f)$ :

Auf $\mathcal{A}(W)$ ist $\omega$ injektiv.

Für Abschnitte $A, B$ ist nämlich $A \subseteq B$ oder $B \subseteq A$, und nach Cantors Isomorphiesatz ist keine Womenge zu einem echten Abschnitt isomorph.

Es folgt $\mathcal{A}^{*}(W) \omega=x>$ und weiter (f).

${ }^{61}$ Dieses Vorgehen bietet sich generell an, um Stabilitäts,,probleme“ zu vermeiden (in 10.2 sind a priori nur (b) und (e) stabil). Insbesondere wenn eindeutige Existenz behauptet wird. Auf der Klassenebene ist Einzigkeit nicht unbedingt stabil. Deshalb wurde in die neue Formulierung des $f$-Kettensatzes auch der "Zusatz" einbezogen. Letzen Endes ist das ganze Thema ein rein ästhetisches; ob eine Eigenschaft eines existenten Objektes in die Existenzaussage einbezogen oder separat als $f f$-Resultat analog $6.7_{f f}$ formuliert wird, ist bezüglich der Konsequenzen gleichgültig.

${ }^{62} \mathrm{Im} 0$-Modus müßte $B$ als linear oder regulär vorausgesetzt werden.

Wohlweislich habe ich von vornherein (schon in Teil I) linear in wohlgeordnet einbezogen, denn linear ist als Oderaussage a priori „nicht“ stabil. 
10.7. Da die Geoklasse $\mathcal{O}$ linear ist und $x \geq$ immer wohlgeordnet, ist auch $\mathcal{O}$ selbst wohlgeordnet: ist $L$ nämlich eine nichtleere Teilklasse und $x \in L$, so ist ein Minimum von $L \cap x \geq$ auch minimal in $L$.

Dann kann aber $\mathcal{O}$ keine Menge sein, denn sonst wäre $W=(\mathcal{O}, \leq) \in \mathcal{W}$ laut (f) doch zu einem echten Abschnitt isomorph.

Daß mit $\mathcal{O}$ auch $\mathcal{C}$ wohlgeordnet ist, ergibt sich aus dem Hilfssatz:

Es existiert die Funktion $\alpha: \mathcal{O} \rightarrow \mathcal{C}$, welche jedem $x \in \mathcal{O}$ die Kardinalität der Grundmenge der Geomenge $x \omega^{\prime}$ zuordnet $\left[x \alpha=\left(x \omega^{\prime}\right)_{1} \chi\right]$, und $\alpha$ ist surjektiv (Wohlordnungssatz).

Und daß mit $\mathcal{O}$ auch $\mathcal{C}$ keine Menge ist, folgt so:

Andernfalls wäre auch die Teilklasse $\mathcal{M}^{\prime}=\mathcal{C} \chi^{\prime}$ von $\mathcal{M}$ eine Menge $\left[\mathcal{M}_{7}\right]$, folglich auch $\mathcal{M}^{\prime} \times$ Rel, damit auch die Teilklasse $\mathcal{W}$ und somit auch $\mathcal{W} \omega=\mathcal{O}$, ein Widerspruch. Zu ,Teilklasse $\mathcal{W}^{\text {“: }}$ Eine zur Grundmenge einer Womenge $W$ gleichmächtige Menge ist Grundmenge einer zu $W$ isomorphen Womenge.

10.8. Die Implikation (c) $\Rightarrow(d)$ ist trivial ( $K$ ist regulär).

Mit $\mathcal{C}$ hätte auch $\mathcal{O}$ kein Maximum [Wohlordnungssatz], und daß $\mathcal{C}$ tatsächlich kein Maximum hat, ergibt sich natürlich aus Cantors berühmtem Satz

$$
|M|<|\mathcal{P}(M)|
$$

über die Potenzmenge einer Menge. Er ist stabil und gilt auch für Klassen $M$ :

Zunächst gilt $\leq$, denn die Abbildung $x \rightarrow\{x\}$ ist injektiv.

Statt lediglich $\neq$ wird mehr nachgewiesen, nämlich $\not$.

Sei also $\alpha: \mathcal{P}(M) \rightarrow M$ injektiv. Nach 5.4 existiert $R \subseteq M$ mit

$$
x \in R \Longleftrightarrow x=U \alpha \Rightarrow x \notin U \text {. }
$$

Betrachte $x=R \alpha$ und beachte $x=U \alpha \Rightarrow U=R$.

Die Aussage $S: \Leftrightarrow x \in R$ genügt der Lügnerregel [1.3(f)], ein Widerspruch.

10.9. Krönender Abschluß ist die Isomorphie der Geoklassen $\mathcal{C}$ und $\mathcal{O}$.

Sie gilt allgemein für Woklassen $K_{1}, K_{2}$, deren echte Abschnitte Mengen sind, sie selbst aber nicht. Dann kann $K_{1}$ nämlich nicht zu einem echten Abschnitt von $K_{2}$ isomorph sein $\left[\mathcal{M}_{8}\right]$. Jetzt müssen wir nur beachten, daß Cantors Isomorphiesatz für Woklassen richtig bleibt: es gilt $K_{1} \nearrow K_{2}$ oder $K_{2} \nearrow K_{1}$.

10.10. Es gibt bis auf Isomorphie genau eine Woklasse $K$ mit den in 10.2 genannten Eigenschaften.

Und ein solches $K$ (regulär!) heiße vom Typ $I N^{*}$. 


\section{Rückschau auf Teil I.}

Im Sinne des in $\S 7$ und 8 ausgeführten bleibt mit einigen Modifikationen alles richtig, in weitem Umfang sogar für Klassen.

Hier und da ist ein $f f$ anzufügen oder eine Formulierung umzugestalten, klarheitshalber, etwa um die Natur eines Satzes als Existenzsatz herauszuarbeiten. So wie bei dem schon in 8.9 umformulierten $f$-Kettensatz, aus dem sich unmittelbar das Zornsche Lemma und der Wohlordnungssatz ergeben, beide für Klassen. Des weiteren ist 8.7 zu beherzigen: Wie ich es schon in $\S 10$ mit $\mathcal{A}$ und $\mathcal{A}^{*}$ gehalten habe, ist in $\operatorname{Min}(X)$ die Funktion Min zu sehen, in $f^{+}(A)$ die Funktion $f^{+}$, bei $G_{>a}$ die Funktion $a \rightarrow G_{>a}$, bei $A^{+}$die Funktion $A \rightarrow A^{+}$.

Ich wiederhole mich: Außer im + -Modus stellt die zur Entwicklung einer mathematischen Theorie gehörige Benennung bestimmter Objekte nichts anderes dar als eine lange Liste existenter (multivariablen) Funktionen. Es sollte genügen, sich dessen bewußt zu sein, im Vertrauen auf 5.6 und 8.5 jede einschlägige „Definition“ unausgesprochen so zu verstehen.

Besonders einfache Verhältnisse liegen beim Satz von Schröder-Bernstein vor [I.6.6]: Er ist ein Existenzsatz, der Beweis findet also im $f f$-Modus statt (sofern nicht im +-Modus), es genügt also I.5.8(a $)_{f f}$, und am Ende ergibt sich $\gamma$ aus dem Fortsetzungssatz 5.8(d). Und auch er gilt für Klassen.

Bei Cantors Isomorphiesatz I.6.4 - jetzt wiederum für Klassen, Woklassen also - wäre jetzt $(\mathrm{b})_{f f}$ zu schreiben, wegen „oder". Und, wie schon früher ausgeführt (in Fußnote 61), auch $(\mathrm{a})_{f f}$, weil die Gleichheitsbeziehung einer Klasse nicht stabil zu sein braucht (da im +-Modus alle Klassen regulär sind, treten solche $f f$ 's nur im 0-Modus auf).

Im Hinblick auf I.6.1 erfordert der Beweis von (b) die Abbildung, welche jedem $A$ sein $\alpha_{A}$ zuordnet. Mehr dazu später.

Die Voraussetzung in I.6.2 ist als Vorgabe einer Abbildung zu verstehen, welche jedem $A$ eine Abbildung $\alpha \rightarrow \alpha^{+}$zuordnet. Und wieder im Hinblick auf I.6.1 erfordert der Beweis eine Abbildung, welche jedem $U$ sein $\alpha_{U}$ zuordnet.

Bei dem eigentlichen Thema "Aufbau des Zahlensystems" geht es um eine Reihe von Existenzsätzen, um die (bis auf Isomorphie eindeutige) Existenz gewisser geordneter Mengen, Gruppen, Ringe und Körper. Alles, was im Hinblick darauf nur dienende Funktion hat, kann man also modulo $f f$ betrachten, und damit findet die gesamte Diskussion de facto im $f f$-Modus statt (wenn nicht von vornherein im + -Modus).

Auch der in Richtung $\mathbb{R}$ entscheidende Satz I.1.4 ist seiner Natur nach ein Existenzsatz: Es existiert eine vollständige Geogruppe $G^{*}$ mit ..., und ein Monomorphismus $\gamma: G \rightarrow G^{*}$ mit $\ldots$. 
Dem genannten Thema liegt eine Voraussetzung zugrunde: Es gibt eine unendliche Menge. Auf der Klassenebene ist diese Voraussetzung redundant: Die injektive, aber nicht surjektive Funktion $\mathcal{P}$ macht die Klasse $\mathcal{M}$ unendlich.

Daraus resultiert eine Pseudozahlenwelt, bestehend aus gewissen geordneten Klassen, Pseudogruppen, -ringen, -körpern. Im ersten Schritt erhält man über die Klassenversion von I.5.9 aus einer unendlichen Klasse eine Geoklasse $W$ vom Typ $I N$, und was alles über entsprechende Geomengen sowie endliche Mengen gezeigt wurde, bleibt auf der Klassenebene gültig.

Im + - wie im $f f$-Modus ist jede einelementige (oder leere) Klasse eine Menge. Per Induktion [I.8.3] folgt das auch für endliche Klassen $K$ :

Wir dürfen $K$ als Woklasse voraussetzen, deren echte Abschnitte Mengen sind. Mit $a=\max (K)$ ist dann $M=K \backslash\{a\}$ eine Menge, und die Potenzmenge $P=\mathcal{P}(M)$ läßt sich ebenfalls wohlordnen. Im Falle $K \nearrow P$ ist $K$ eine Menge, da gleichmächtig mit einer Menge (einer Teilmenge von $P$ ), und im Falle $P \nearrow K$ ist $P \simeq K$ oder $P \nearrow M$. Letzteres widerspricht aber Cantors Satz I.10.8.

Um in gewohnter Weise frei endliche Summen bzw. Produkte in kommutativen Halbgruppen bilden zu können, bedarf es nicht nur des Satzes in I.8, sondern (im $f f$-Modus) einer 2-variablen Funktion $\Pi$, welche $W$ und $H$ ein $\pi$ zuordnet. Diese wird gemäß 5.7 auf $A=\mathcal{E} \times \mathcal{H}$ etabliert, wobei $\mathcal{E}$ die Klasse der endlichen Mengen ist und $\mathcal{H}$ die Klasse der kommutativen Halbgruppen.

Stattdessen läßt sich auch routinemäßig mit 8.5 arbeiten.

Die in I.7 beschriebenen Beziehungen zwischen (un)endlichen Mengen und einer Womenge $W$ vom Typ $I N$ gelten analog für Mengen bzw. Unmengen $\left(\right.$ Klassen außerhalb $\mathcal{M}$ ) und eine Woklasse $W^{*}$ vom Typ $\mathbb{I}^{*}[10.10]$ :

Eine Womenge ist zu genau einem echten Abschnitt von $W^{*}$ isomorph.

Genau dann ist eine Klasse eine Menge, wenn sie zu einem echten Abschnitt von $W^{*}$ gleichmächtig ist.

Genau dann ist eine Woklasse eine Unmenge, wenn sie einen zu $W^{*}$ isomorphen Abschnitt hat.

Genau dann ist eine Klasse eine Unmenge, wenn sie eine zu $W^{*}$ gleichmächtige Teilklasse hat.

Es gibt einen gewichtigen Unterschied: Eine endliche Menge ist nur zu einem einzigen echten Abschnitt von $W$ gleichmächtig.

Eine Woklasse ist genau dann endlich, wenn sie ein Maximum - und jedes nichtminimale Element einen Vorgänger hat. Die Vereinigung der endlichen Abschnitte von $W^{*}$ ist daher vom Typ $I N$ (mit $A$ ist auch $A^{+}=A \cup\left\{f^{+}(A)\right\}$ endlich). Sie ist in allen unendlichen Abschnitten enthalten, ist also ihr Schnitt, außerdem der einzige Abschnitt vom Typ $I N$. 
Wir kehren jetzt zu I.6.1 zurück, vor allem um besser mit den beim Übergang auf die Klassenebene auftretenden Problemen und ihren Lösungsmöglichkeiten vertraut zu werden. Wie oben angedeutet, wird implizit eine Abbildung $\lambda$ vorausgesetzt, die jedem $A \in \mathcal{B}$,sein $\alpha_{A}$ " zuordnet.

Somit wäre $\alpha_{A}=A \lambda$ mit $\lambda: \mathcal{B} \rightarrow \gamma$ wobei $A \gamma=\mathcal{F}(A, M) \in \mathcal{K}$.

In einer Anwendungssituation wie I.6.2 müßte zuerst die Existenz von $\gamma$ : $\mathcal{B} \rightarrow \mathcal{K}$ bewiesen werden, die von $\lambda$ ergäbe sich dann routinemäßig aus 5.6.

Auf der Mengeneben wäre $\gamma: \mathcal{B} \rightarrow \mathcal{M}$, und die Existenz daher kein Problem, wie wir schon in 6.4 gelernt haben.

Auf der Klassenebene legt der Fortsetzungssatz die folgende Lösung nahe:

Arbeite von vornherein mit Abbildungen $\alpha_{A}=A \lambda$ von $V$ statt $A$ in $M$.

Dann vereinfacht sich $\lambda$ zu $\lambda: V \rightarrow \mathcal{F}(V, M)$ und $\gamma$ tritt nicht auf.

Die Existenz von $\alpha$ (in I.6.1 neu) ergibt sich dann genau wie vorher aus 5.6, und zwar ist $\alpha$ eine Auswahlfunkion zu der Abbildung $\sigma: V \rightarrow \mathcal{P}(M)$ mit

$$
x \sigma=\left\{y \in M \mid \exists A \in \mathcal{B} \text { mit } x \in A \text { und } y=x \alpha_{A}\right\} .
$$

Man diskutiere I.6.2 analog I.6.1, also mit $\mathcal{F}_{A}=\mathcal{F}(W, M)$.

Statt einen Satz $S$ im Beweis eines Satzes $T$ anzuwenden, kann es zweckmäßig sein, auf $S$ ganz zu verzichten und den Beweis für $S$ soweit nötig in den für $T$ zu integrieren, und zwar um damit die Komplexität zu verringern, störende Existenzfragen für Abbildungen zu eliminieren. Beispielsweise läßt sich $6.1 \mathrm{im}$ Beweis von I.6.4 $(\mathrm{b})_{f f}$ folgendermaßen eliminieren:

Zunächst existiert $\sigma: W \rightarrow W^{\prime}$ mit

$$
x \sigma=\left\{y \in W^{\prime} \mid \exists U \in \mathcal{B} \text { und } \mu: U \nearrow W^{\prime} \text { mit } x \in U \text { und } y=x \mu\right\},
$$

und eine Auswahlfunktion $\alpha: W \rightarrow W^{\prime}$ setzt jedes $\mu: U \nearrow W^{\prime}$ fort.

Wie stellen sich die Dinge im +-Modus dar? Als erstes stellen wir fest, daß die dortigen Gegebenheiten mit der Formulierung „sei gegeben“ in I.6.1 voll und ganz harmonieren, denn im +-Modus ist zwar alles mögliche explizit gegeben, aber nicht durch jeweils eine Funktion analog 8.5, sondern eine Kombination von Funktionen, was sich nicht allgemein als Aussage formulieren läßt. Für die Zweckmäßigkeit der gegebenen Formulierung ist also in Kauf zu nehmen, daß sie nur meta-mathematisch zu verstehen ist, d.h. grundsätzlich als Anweisung, wie in einer Anwendungssituation zu verfahren ist. Um etwas konkreter $\mathrm{zu}$ werden: Im Beweis von I.6.2 bzw. I.6.4(b) läßt sich $\alpha_{A}$ bzw. $\alpha_{U}$ explizit als $\ell\{\delta \in \mathcal{F}(A, M) \mid \ldots\}$ bzw. $\ell\left\{\delta \in \mathcal{F}\left(U, W^{\prime}\right) \mid \ldots\right\}$ angeben. 


\section{Mengensysteme.}

Man lese folgendes im +- oder $f f$-Modus, oder hier und da modulo $f f$.

Ein Mengensystem ist eine Klasse von Klassen analog $\mathcal{M}$, ist also ein Element des Bereiches $\mathcal{P}(\mathcal{K})$ aller Klassen von Klassen, auch Quasiteilklassen von $\mathcal{K}$ genannt. Dieser existiert nach Axiom I als Teilbereich von $\mathcal{K}$.

Die Mengensysteme bilden einen Teilbereich $\mathcal{P}_{m}(\mathcal{K})$ von $\mathcal{P}(\mathcal{K})$.

Ein (in des Wortes doppelter Bedeutung) nichtleerer Schnitt von Mengensystemen ist wiederum ein Mengensystem, und man sieht leicht, daß die endlichen Mengen ein Mengensystem bilden, etwa $\mathcal{E}$ wie oben. Außerdem ist jede endliche Klasse eine Menge. Somit ist $\mathcal{E}$ der Schnitt aller Mengensysteme. Ein Mengensystem heiße vollständig, wenn es eine unendliche Klasse enthält.

Existiert eine unendliche Menge, dann auch ein $W$ wie oben, und dasselbe gilt für jedes Mengensystem. Jede mit $W$ gleichmächtige Klasse ist eine Menge, und jede Woklasse vom Typ $I N$ ist zu $W$ isomorph. Also ist auch der Schnitt $\mathcal{V}$ aller vollständigen Mengensysteme vollständig.

In welchem Sinne können wir hier und weiter oben überhaupt von einem „Schnitt" reden? Jedenfalls nicht via $\bigcap$, denn die Klasse $\mathcal{P}_{m}(\mathcal{K})$ ist nicht in Sicht, und die Klasse $\mathcal{P}(\mathcal{K})$ existiert definitiv nicht [5.9(d)].

Man könnte natürlich die Klasse aller Mengensysteme einfach postulieren. Das ist aber nicht nötig: Ist $\mathcal{A}$ ein Bereich von Mengensystemen, allgemeiner ein Teilbereich von $\mathcal{P}(\mathcal{K})$, so existiert zu $A \in \mathcal{A}$ der Schnitt gemäß 5.4 bzw. 7.2 als Teilklasse von $A$.

Dies erklärt nicht nur die in 7.3 erwähnte Existenz des Schnittes $A \cap B$ von $A, B \in \mathcal{P}(\mathcal{K})$; es macht auch klar, daß $A \cap B$ nur relativ zu $A$ wie zu $B$ „gegeben“ ist, im Unterschied zum Schnitt zweier Teilklassen einer Klasse.

Dem entsprechend existiert, falls $\mathcal{M}$ vollständig ist, $\mathcal{V}$ im + -Modus stark als Teilklasse von $\mathcal{M}$. Und allgemein existiert $\mathcal{V}$ wenn es überhaupt ein vollständiges Mengensystem gibt, und ist dann das kleinste vollständige Mengensystem.

Der Bereich $\mathcal{P}_{m}(\mathcal{K})$ ist bezüglich $\subseteq$ linear geordnet; liegt nämlich $A \in \mathcal{M}$ nicht in einem Mengensystem $\mathcal{L}$, so folgt $|A| \not \leq|B|$ für jedes $B \in \mathcal{L}$, also $|B| \leq A$ und damit $B \in \mathcal{M}$. Eine $\subseteq$ entsprechende Relation liegt allerdings nicht vor.

Einem Mengensystem $\mathcal{L} \subseteq \mathcal{M}$ entspricht ein Abschnitt $U$ von $W^{*}$ ohne Maximum. Seine echten Abschnitte sind diejenigen Abschnitte von $W^{*}$, welche zu einem $B \in \mathcal{L}$ gleichmächtig sind; $U$ ist also die Vereinigung dieser Abschnitte und verhält sich zu $\mathcal{L}$ wie $W^{*}$ zu $\mathcal{M}$. 



\section{LITERATUR}

1. Bernays, P.: Hilberts Untersuchungen über die Grundlagen der Arithmetik.

Hilberts Ges. Werke III (Springer, Berlin 1935), 197-216.

2. - - Erwiderung auf die Note von Herrn Aloys Müller: „Über Zahlen als Zeichen“.

Math. Ann. 90 (1923), 159-163.

3. - - und Fraenkel, A. A.: Axiomatic set theory.

North Holland Publishing Co., Amsterdam 1958.

4. Bolzano, B.: Philosophie der Mathematik oder Beiträge zu einer begründeteren Darstellung der Mathematik. Verlag Carl Widtmann, Prag 1810.

5. Cantor, G.: Beiträge zur Begründung der transfiniten Mengenlehre.

Math. Ann. 46 (1895), 481-512.

6. Dedekind, R.: Stetigkeit und irrationale Zahlen. Vieweg, Braunschweig 1872.

7. - - Was sind und was sollen die Zahlen? Vieweg, Braunschweig 1888.

8. Fraenkel, A.: Die Gleichheitsbeziehung in der Mengenlehre.

J. f. Math. 157 (1926), 79-81.

9. - - Untersuchungen über die Grundlagen der Mengenlehre.

Math. Z. (1925), 250-273.

10. Hilbert. D.: Neubegründung der Mathematik. Erste Mitteilung.

Abh. Math. Sem. Univ. Hbg. 1 (1922), 157-177).

11. - - Über das Unendliche. Math. Ann. 95 (1925), 161-190.

12. - - Die Grundlegung der elementaren Zahlenlehre.

Math. Ann. 104 (1931), 485-494.

13. - - Beweis des Tertium non datur. Nachr. Ges. Wiss. Göttingen (1931).

14. Kneser, H.: Das Auswahlaxiom und das Lemma von Zorn.

Math. Z. 96, 62-63 (1967).

15. Kronecker, L.: Über den Zahlbegriff. J. f. Math. 101 (1887), 337-355.

16. Müller, A.: Über Zahlen als Zeichen. Math. Ann. 90 (1923), 153-158.

17. Schönfinkel, M.: Über die Bausteine der mathematischen Logik.

Math. Ann. 92 (1924), 305-316.

18. Wittenberg, A. I.: Vom Denken in Begriffen. Birkhäuser, Basel 1957.

[Untertitel:] Mathematik als Experiment des reinen Denkens.

19. Zermelo. E.: Neuer Beweis für die Möglichkeit einer Wohlordnung. Math. Ann. 65 (1908), 107-128.

20. - - Untersuchungen über die Grundlagen der Mengenlehre. I Math. Ann. 65 (1908), 261-281.

21. - - Über den Begriff der Definitheit in der Axiomatik. Fund. Math. 14 (1929), 339-344.

22. - - Über Grenzzahlen und Mengenbereiche.

[Untertitel:] Neue Untersuchungen über die Grundlagen der Mengenlehre.

Fund. Math. 16 (1930), 29-47. 

Dieses Buch ist als eine Neubegründung der Mathematik zu verstehen. Mathematische Grundkenntnisse genügen zum Verständnis. In logischer Hinsicht wird nur der vertraute Umgang mit den Worten und, oder, wenn/dann vorausgesetzt. Logische Fachkenntnisse sind somit nicht nötig, eher hinderlich.

Die Grundbegriffe Objekt, Bereich, Abbildung sind rein sprachlicher Natur: Ein Bereich erlaubt, von seinen Elementen (Objekte) zu reden, eine Abbildung $\alpha$ von dem „Bild“ $x \alpha$ (ein Objekt) eines Objektes $x$. Und selbstverständlich sind Bereiche wie Abbildungen auch Objekte.

Einhergehend mit Negation wird Existenz eingeführt, Gleichheit dann im Zusammenhang mit dem Begriff einer Klasse (Bereich plus Äquivalenzrelation), und schließlich wird die Klasse aller Mengen vorausgesetzt.

Das Standard-Vorlesungsthema Aufbau des Zahlensystems wird vorweg auf althergebrachter Grundlage behandelt (Mengen und Abbildungen im Sinne Dedekinds), in dem vertrauten Rahmen der real existierenden Mathematik also, aber nicht auf altvertraute Weise.

Der entsprechende, um einiges angereicherte Teil I des Buches ist daher von unabhängigem Interesse, wie auch Teil II mit historischen und mathematikphilosophischen Erörterungen.

\section{Logos Verlag Berlin}

